\title{
Consistent treatment of rapidity divergence in soft-collinear effective theory
}

\author{
Junegone Chay ${ }^{a}$ and Chul $\mathrm{Kim}^{b}$ \\ ${ }^{a}$ Department of Physics, Korea University, \\ Seoul 02841, Korea \\ ${ }^{b}$ Institute of Convergence Fundamental Studies and School of Liberal Arts, \\ Seoul National University of Science and Technology, \\ Seoul 01811, Korea \\ E-mail: chay@korea.ac.kr, chul@seoultech.ac.kr
}

ABSTRACT: In soft-collinear effective theory, we analyze the structure of rapidity divergence due to the collinear and soft modes residing in disparate phase spaces. The idea of an effective theory is applied to a system of collinear modes with large rapidity and soft modes with small rapidity. The large-rapidity (collinear) modes are integrated out to obtain the effective theory for the small-rapidity (soft) modes. The full SCET with the collinear and soft modes should be matched onto the soft theory at the rapidity boundary, and the matching procedure becomes exactly the zero-bin subtraction. The large-rapidity region is out of reach for the soft mode, which results in the rapidity divergence. The rapidity divergence in the collinear sector comes from the zero-bin subtraction, which ensures the cancellation of the rapidity divergences from the soft and collinear sectors. In order to treat the rapidity divergence, we construct the rapidity regulators consistently for all the modes. They are generalized by assigning independent rapidity scales for different collinear directions. The soft regulator incorporates the correct directional dependence when the innate collinear directions are not back-to-back, which is discussed in the $N$-jet operator. As an application, we consider the Sudakov form factor for the back-to-back collinear current and the soft-collinear current, where the soft rapidity regulator for a soft quark is developed. We extend the analysis to the boosted heavy quark sector and exploit the delicacy with the presence of the heavy quark mass. We present the resummed results of large logarithms in the form factors for various currents with the light and the heavy quarks, employing the renormalization group evolution on the renormalization and the rapidity scales.

KeYwords: Effective Field Theories, Perturbative QCD, Resummation

ARXIV EPRINT: 2008.00617 


\section{Contents}

1 Introduction 1

2 Rapidity divergence and the zero-bin subtraction 4

2.1 Effective theory approach to treating the rapidity divergence 4

$\begin{array}{lll}2.2 & \text { Collinear contribution and the zero-bin subtraction } & 6\end{array}$

$\begin{array}{lll}2.3 & \text { Rapidity regulator in the soft sector } & 7\end{array}$

3 Sudakov form factor for the back-to-back current 9

3.1 Soft one-loop contribution to the back-to-back collinear current 9

$\begin{array}{ll}3.2 & \text { Factorization of the Sudakov form factor } \\ \end{array}$

$\begin{array}{lll}3.3 & \text { On-shell regularization with a massless gluon } & 12\end{array}$

$\begin{array}{lll}3.4 & \text { Soft contributions to timelike processes } & 14\end{array}$

4 The $N$-jet operator $\quad 16$

5 Sudakov form factor from the soft-collinear current 20

6 Sudakov form factor involving heavy quarks 23

6.1 Heavy-to-heavy form factor 24

6.1.1 Factorization with $Q \gg m \sim \mu \quad 24$

$\begin{array}{lll}\text { 6.1.2 Factorization with } Q \gg m \gg \mu & 25\end{array}$

6.2 Heavy-to-light form factor 30

$\begin{array}{lll}7 & \text { Conclusions } & 32\end{array}$

A Resummation of large logarithms in Sudakov form factor 33

A.1 Sudakov form factor for the light back-to-back current 34

A.2 Sudakov form factor for the soft-collinear current 38

$\begin{array}{lll}\text { A.3 Sudakov form factor for the heavy-to-heavy current } & 39\end{array}$

A.4 Sudakov form factor for the heavy-to-light current 40

B One-loop calculations in the boosted heavy quark sector 41

B.1 Collinear one-loop calculation with the soft zero-bin mode $p_{s}^{\mu} \sim(m, m, m) \quad 41$

B.2 Csoft one-loop calculation with the usoft zero-bin mode $p_{u s}^{\mu} \sim \zeta(m, m, m) \quad 42$ 


\section{Introduction}

Effective field theories enable us to understand important physics by extracting relevant ingredients and disregarding the unnecessary remainder. Soft-collinear effective theory (SCET) [1-4] is an effective theory for QCD, which is appropriate for high-energy processes with energetic particles immersed in the background of soft particles. We pick up the collinear and soft modes to describe high-energy processes, and all the other modes are either integrated out or neglected. The degrees of freedom in the effective theory are classified by the phase spaces they reside in.

Since there are various modes in different phase spaces in SCET, it possesses an additional divergence known as the rapidity divergence $[5,6]$ in addition to the traditional ultraviolet (UV) and infrared (IR) divergences. A boundary in the phase space to separate collinear and soft modes results in the rapidity divergence because these modes are constrained in different phase spaces. In full QCD, there is no rapidity divergence because there is no kinematic constraint. Therefore in SCET there may be rapidity divergence in each sector, but when we sum over all the contributions from different sectors, there should be no rapidity divergence. This is a good consistency check for the effective theory. In this respect, the rapidity divergence seems to be an artifact in SCET by dissecting the phase space, which disappears in the total contribution. But it gives a richer structure of the effective theory and we can obtain deeper understanding of underlying physics.

Here we consider a system of the collinear and soft modes with the same offshellness, in which they are distinguished by their rapidities. The soft modes have small rapidity, while the collinear modes have large rapidity. For the factorization into the soft and collinear parts in SCET, the central idea is to apply the conventional effective theory approach that is widely used for separating long- and short-distance physics.

We first construct an effective theory for the soft mode out of the full SCET. The full SCET contains both collinear and soft modes, while the soft theory contains only soft modes with small rapidity. By requiring that physics be the same near the rapidity boundary, the full SCET with both modes is matched onto the soft theory, and produces the Wilson coefficient. The Wilson coefficient is obtained by subtracting the contribution of the soft theory from that of the full SCET. In the matching near the boundary, the contribution of the soft theory to be subtracted is exactly the zero-bin contribution in SCET. Therefore the collinear contribution with the zero-bin subtraction [7] can be interpreted as the Wilson coefficients for the soft theory.

Note that the soft mode cannot resolve the large rapidity region. And we expect that there appears the rapidity divergence as the soft momentum approaches the rapidity boundary. Suppose that the collinear mode beyond the boundary is $n$-collinear. Then the rapidity divergence in the soft sector arises as the momentum component $\bar{n} \cdot k \rightarrow \infty$ (and $n \cdot k \rightarrow 0$ ). On the other hand, the naive collinear contribution before the zero-bin subtraction has no corresponding rapidity divergence since $\bar{n} \cdot k$ is bounded from above. However the true collinear part after the matching, which includes the zero-bin subtraction, contains the rapidity divergence with the same origin as the soft part. Therefore the rapidity divergences in the collinear and the soft sectors have the opposite sign, which ensures the cancellation of the rapidity divergence when both are combined. 
The naive collinear part might contain another type of the rapidity divergence as $\bar{n} \cdot k \rightarrow 0$. But that region is shared with the soft part and this type of rapidity divergence is cancelled in the matching. And the true rapidity divergence with $\bar{n} \cdot k \rightarrow \infty$ in the collinear sector is recovered by the zero-bin subtraction, similar to the pullup mechanism [8,9]. As a result, the soft-collinear factorization with the zero-bin subtraction is identified as the matching of the full SCET onto the soft part, and the collinear part can be considered as the matching coefficient describing the large-rapidity region.

The main issue of this paper is to implement this idea for the consistent treatment of the rapidity divergence in SCET. The first step is to establish a proper method for regulating the rapidity divergences in the collinear and the soft sectors. We prescribe the rapidity regulators in both sectors, originating from the same source.

In addition to the conventional regularization method $[5,6]$, there have been many suggestions to regulate the rapidity divergence, such as the use of the Wilson lines off the lightcone [10], the $\delta$-regulator [11, 12], the analytic regulator [13], the exponential regulator [14], and the pure rapidity regulator [15], to name a few. The gauge invariance and the consistency in power counting to all orders have also been recent issues in selecting appropriate rapidity regulators $[6,15,16]$. Here we present a new prescription for the rapidity regulator based on where the rapidity divergence arises, as discussed earlier. It may look like an addition to a big list of regulators, but we hope to convince the reader that our prescription is on a sound physical basis.

The construction of the rapidity regulators is interwoven between the collinear and the soft sectors, and let us first look at the Wilson lines in SCET. The emission of collinear or soft gluons from collinear, energetic particles is eikonalized and exponentiated to all orders to yield the collinear and soft Wilson lines by integrating out large offshell modes. The collinear and the soft Wilson lines in the lightlike $n$ direction are written as [3]

$$
W_{n}=\sum_{\text {perm. }} \exp \left[-g \frac{\bar{n} \cdot A_{n}}{\bar{n} \cdot \mathcal{P}}\right], S_{n}=\sum_{\text {perm. }} \exp \left[-g \frac{n \cdot A_{s}}{n \cdot \mathcal{P}}\right]
$$

where $A_{n}\left(A_{s}\right)$ is the collinear (soft) gauge field. The lightlike vectors $n$ and $\bar{n}$ satisfy $n^{2}=\bar{n}^{2}=0, n \cdot \bar{n}=2$, and $\bar{n} \cdot \mathcal{P}(n \cdot \mathcal{P})$ is the operator extracting the incoming momentum component in the $n(\bar{n})$ direction.

Note that the nature of the eikonalization is different in both cases. For the collinear Wilson line $W_{n}$, we consider the emission of the $n$-collinear gluons from all the remaining parts except the $n$-collinear particle in consideration, that is, from the $\bar{n}$-collinear field in a back-to-back current or from the heavy quark in a heavy-to-light current. For the $N$-jet operator, we consider the $n$-collinear gluons emitted from all the collinear particles except the $n$-collinear particle. Whatever the sources are, when the intermediate states are integrated out, and the leading terms are taken, we obtain the collinear Wilson line which depends on $\bar{n} \cdot \mathcal{P}$. On the other hand, the soft Wilson line $S_{n}$ is obtained by the emission of soft gluons from an $n$-collinear field, and the intermediate states are integrated out. Note that the source of the gluon emission is different. Therefore to extract the rapidity divergences in the soft and the collinear sectors correctly, we should trace the $n$-collinear gluons in the collinear sector, and also the soft gluons from the same source as in the 
collinear sector, and take the $n$-collinear limit. Simply put, the rapidity matching does not happen between $W_{n}$ and $S_{n}$, but happens between $W_{n}$ and $S_{\bar{n}}$ for the back-to-back current.

Let us take an example of the back-to-back current $\bar{\xi}_{\bar{n}} W_{\bar{n}} S_{\bar{n}}^{\dagger} \gamma^{\mu} S_{n} W_{n}^{\dagger} \xi_{n}$, which will be generalized later, and consider a soft gluon emitted from the soft Wilson line, not from $S_{n}$, but from $S_{\bar{n}}^{\dagger}$. In order to consider the matching with the $n$-collinear sector, in which the $n$-collinear gluon is emitted from the $\bar{n}$-collinear sector, we take the limit in which the component $\bar{n} \cdot k$ of the soft momentum becomes large, compared to other components with $k_{\perp}^{2}$ fixed. And it is taken to infinity in the soft sector because the large scale is beyond reach of the soft particles. Therefore the region $\bar{n} \cdot k \rightarrow \infty$ is where the possible rapidity divergence occurs and we apply the rapidity regulator to extract it. We can choose the rapidity regulator in the form $(\nu / \bar{n} \cdot k)^{\eta}$, where $\nu$ is the rapidity scale introduced and the rapidity divergence appears as poles in $\eta$. In order to be consistent, we also choose the same rapidity regulator in the $n$-collinear sector since the rapidity region modified by the regulator should be the same in the overlapping region.

We can also include the collinear currents which are not back-to-back, or even the $N$-jet operator in which there are well-separated $N$ collinear directions. We emphasize that the same rapidity regulator should be employed both in the collinear and soft sectors. When the rapidity divergence in one collinear sector is to be matched, the soft gluons emitted from other collinear directions are selected and the collinear limit in the given collinear direction is taken. In this process, the directional dependence in the soft sector is correctly produced. Furthermore, we can assign a different rapidity scale to each collinear direction if there is a hierarchy of scales in different directions. The total contribution is free of every rapidity divergence associated with each collinear direction. The rapidity regulator for the soft-collinear current can be also consistently constructed using this method.

Our analysis on the rapidity divergence can be extended to the boosted heavy quark sector. Here the energy of the heavy quark, $Q$, is still much larger than the quark mass $m$. Hence the decoupling of the soft interactions from the heavy quark can be implemented in the same manner as in the case of the light quark. Since the origin of the rapidity divergence is soft dynamics, the divergence in the heavy quark sector also comes from the zero-bin subtraction. This is another new observation we make. We consider the resummation of large logarithms in the Sudakov form factor for the heavy-to-heavy current. Like the light quark case, the rapidity scale evolution is necessary. And it enables us to fully resum the large logarithms of $Q / m$.

The structure of the paper is as follows: in section 2, we discuss the idea of applying an effective theory to a system with the collinear and soft modes, and explain that the rapidity divergence in the collinear part comes from the matching procedure that corresponds to the zero-bin subtraction. And we show how to set up the rapidity regulator with the zero-bin subtraction in the collinear sector. The soft rapidity regulator is defined by employing the same principle for the collinear sector, but with the appropriate expression for the soft Wilson line. In section 3, the Sudakov form factor is analyzed for the back-to-back current. We suggest how to implement the rapidity regulator in the $N$-jet operator in section 4 . In section 5, we consider the Sudakov form factor for the soft-collinear current, which is compared to the result by boosting the back-to-back current. In section 6 , our analysis is 
extended to the heavy-to-heavy and heavy-to-light currents, in which the heavy quark mass sets another hierarchy in factorizing the form factors. Finally we conclude and describe the outlook in section 7. In appendix A, we resum the large logarithms in the Sudakov form factor for various currents using the renormalization group ( $R G$ ) equation with respect to the renormalization and rapidity scales. The evolutions and the resummation are performed to next-to-leading logarithmic (NLL) accuracy. In appendix B, we show the details of the calculations for extracting the rapidity divergences in the boosted heavy quark sector.

\section{Rapidity divergence and the zero-bin subtraction}

We start with the matrix element of the back-to-back collinear current in SCET

$$
V_{\mathrm{SCET}}^{\mu}=\left\langle p\left|\bar{\xi}_{n} W_{n} \tilde{S}_{n}^{\dagger} \gamma_{\perp}^{\mu} S_{\bar{n}} W_{\bar{n}}^{\dagger} \xi_{\bar{n}}\right| p^{\prime}\right\rangle
$$

where $p$ and $p^{\prime}$ are the on-shell momenta of the collinear quarks in the $n$ - and $\bar{n}$-directions respectively. The soft Wilson lines $\tilde{S}_{n}\left(S_{\bar{n}}\right)$ are present by redefining the collinear fields, which results in the decoupling of the soft interactions. The notations follow the convention used in ref. [17]. In higher-order corrections, the UV, IR, and rapidity divergences are produced, in which the UV and the IR divergences are regulated by dimensional regularization. If we introduce a nonzero gluon mass $M$, the IR divergence is regulated by the mass. However, a new regulator is needed to regularize the rapidity divergence.

The widely-used rapidity regulator has been suggested in refs. [5, 6], by modifying the original collinear and soft Wilson lines as

$$
W_{n}=\sum_{\text {perms }} \exp \left[-\frac{g}{\bar{n} \cdot \mathcal{P}} \frac{\nu^{\eta}}{\left|\bar{n} \cdot \mathcal{P}_{g}\right|^{\eta}} \bar{n} \cdot A_{n}\right], S_{n}=\sum_{\text {perms }} \exp \left[-\frac{g}{n \cdot \mathcal{P}} \frac{\nu^{\eta / 2}}{\left|2 \mathcal{P}_{g 3}\right|^{\eta / 2}} n \cdot A_{s}\right] .
$$

Here the rapidity scale $\nu$ is introduced and the rapidity divergence appears as poles in $\eta$. As described in Introduction, we use the rapidity regulator of the form $(\nu / \bar{n} \cdot k)^{\eta}$ for the $n$-collinear sector, which is the same as the prescription in eq. (2.2) for $W_{n}$. Then we construct the soft rapidity regulator to be consistent with the collinear rapidity regulator. This is applied to $S_{\bar{n}}$. Therefore the soft rapidity regulator is different from that in eq. (2.2), and needs further explanation.

\subsection{Effective theory approach to treating the rapidity divergence}

Let us first consider the general argument in treating the rapidity divergence. In $\mathrm{SCET}_{\mathrm{II}}$ where the collinear and the soft modes have the same offshellness, we distinguish these modes by their rapidities. In radiative corrections, there appears the integral of the form

$$
I=\int_{\mu_{L}}^{p_{+}} \frac{d k_{+}}{k_{+}}=\int_{\mu_{L}}^{\Lambda} \frac{d k_{+}}{k_{+}}+\int_{\Lambda}^{p_{+}} \frac{d k_{+}}{k_{+}}
$$

where $p_{+}$is a hard momentum, and $\mu_{L}$ is a soft scale. In the final expression, the integral is divided into the soft and collinear integrals by an arbitray scale $\Lambda$ which separates the soft and collinear regions. 
In the spirit of the zero-bin contribution, the integral $I$, by rearranging the second term, can be written as

$$
I=\int_{\mu_{L}}^{\Lambda} \frac{d k_{+}}{k_{+}}+\left(\int_{0}^{p_{+}} \frac{d k_{+}}{k_{+}}-\int_{0}^{\Lambda} \frac{d k_{+}}{k_{+}}\right) .
$$

The first term in the parenthesis is the naive collinear contribution, and the second term is the zero-bin contribution. We clearly see that double counting is avoided in the collinear part. And if there is any divergence in the naive collinear contribution as $k_{+} \rightarrow 0$, it is removed by the zero-bin subtraction. It guarantees the factorization to secure the independence of the collinear sector as stressed in ref. [16]. The cutoff $\Lambda$ appears in the soft integrals, and in the zero-bin contribution. And it can be taken to infinity as far as the soft modes are concerned. Then with the rapidity regulator, eq. (2.4) can be expressed as ${ }^{1}$

$$
I=\nu^{\eta} \int_{\mu_{L}}^{\infty} \frac{d k_{+}}{k_{+}^{1+\eta}}+\left(\nu^{\eta} \int_{0}^{p_{+}} \frac{d k_{+}}{k_{+}^{1+\eta}}-\nu^{\eta} \int_{0}^{\infty} \frac{d k_{+}}{k_{+}^{1+\eta}}\right) .
$$

The soft and collinear parts in eq. (2.5) are given as

$$
\begin{aligned}
& I_{S}\left(\mu_{L}, \nu\right)=\nu^{\eta} \int_{\mu_{L}}^{\infty} \frac{d k_{+}}{k_{+}^{1+\eta}}=\frac{1}{\eta}+\ln \frac{\nu}{\mu_{L}}, \\
& I_{C}\left(p_{+}, \nu\right)=\nu^{\eta} \int_{0}^{p_{+}} \frac{d k_{+}}{k_{+}^{1+\eta}}-\nu^{\eta} \int_{0}^{\infty} \frac{d k_{+}}{k_{+}^{1+\eta}}=-\frac{1}{\eta}-\ln \frac{\nu}{p_{+}} .
\end{aligned}
$$

The rapidity divergence and the $\nu$ dependence in the soft and collinear sectors cancel when they are combined. But the evolutions with respect to the rapidity scale $\nu$ to $\mu_{L}$ for the soft sector and to $p_{+}$for the collinear sector are necessary for the resummation of the large logarithms in $p_{+} / \mu_{L}[5,6]$.

Note that the separation into the soft and collinear parts in eq. (2.5) is similar to separating the long- and short-distance physics in effective theories. The effective theory at lower energy is matched at the cutoff scale to the full theory, yielding the Wilson coefficients. The same mechanism applies to eq. (2.5). Consider all the modes with the same offshellness, and there is a cutoff rapidity which distinguishes the soft modes with small rapidity and the collinear modes with large rapidity. We match the two regions near the cutoff, which is accomplished by the zero-bin subtraction. It yields the Wilson coefficients, which corresponds to the collinear contribution in this case, when the contributions from the large rapidity (collinear) region and the small rapidity (soft) region are matched at the boundary. We emphasize that the idea of an effective theory is applied to the description of the rapidity divergence, though we do not explicitly construct an effective soft theory.

As a consequence, the rapidity divergence arises entirely due to the fact that the soft part cannot describe large-rapidity physics. And the collinear part contains the divergence

\footnotetext{
${ }^{1}$ Some readers may wonder if the pole $1 / \eta$ can (mathematically) regularize the pole at $k_{+}=0$ as well in the zero-bin contribution in eq. (2.5). But the purpose of the $\eta$-regulator is to capture the divergence as $k_{+} \rightarrow \infty$, meaning that we take $\eta$ to be slightly positive, i.e., $\eta=+0$. It does not regularize the divergence for $k_{+}=0$. We may introduce another $\eta^{\prime}=-0$ to regulate the divergence as $k_{+} \rightarrow 0$ as in pure dimensional regularization. However, the divergence for $k_{+}=0$ cancels and we employ the regulator only for $k_{+} \rightarrow \infty$.
} 
(a)

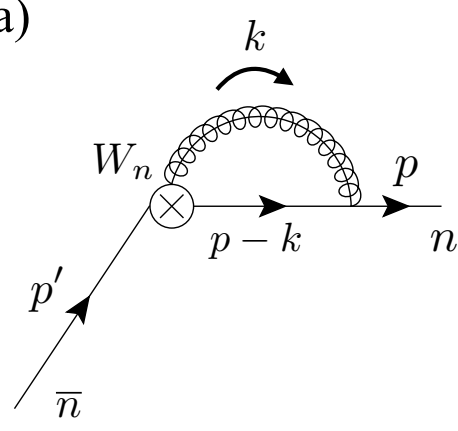

(b)

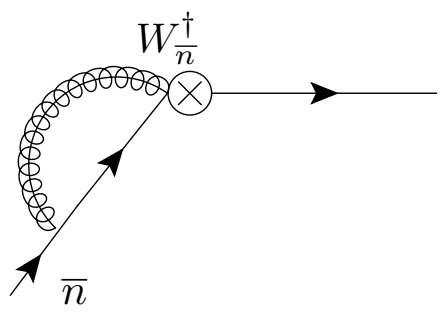

(c)

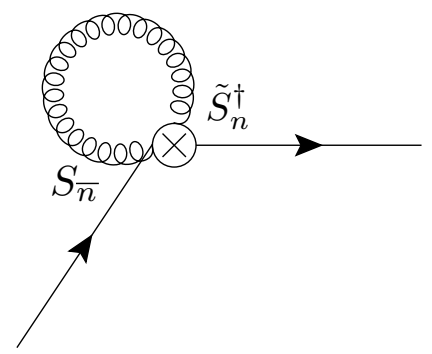

Figure 1. Feynman diagrams for the back-to-back collinear current at one loop.

through matching onto the soft theory with small rapidity, i.e., the zero-bin subtraction. There is always one-to-one correspondence for the rapidity divergences between the soft and the collinear sectors.

\subsection{Collinear contribution and the zero-bin subtraction}

The matrix element in eq. (2.1) is factorized into the $n-, \bar{n}$-collinear and the soft parts. Let us first consider the $n$-collinear contribution at one loop. The corresponding Feynman diagram is shown in figure 1 (a). The naive collinear contribution is given by

$$
\tilde{M}_{n}=2 i g^{2} C_{F} \mu \frac{2 \epsilon}{\mathrm{MS}} \nu^{\eta} \int \frac{d^{D} k}{(2 \pi)^{D}} \frac{\bar{n} \cdot(p-k)}{\left(k^{2}-2 p \cdot k+i \varepsilon\right)\left(k^{2}-M^{2}+i \varepsilon\right)(\bar{n} \cdot k)^{1+\eta}},
$$

where $\mu \frac{2 \epsilon}{\mathrm{MS}}=\left(\mu^{2} e^{\gamma_{\mathrm{E}}} / 4 \pi\right)^{\epsilon}$ in the $\overline{\mathrm{MS}}$ scheme and we employ the rapidity regulator in eq. (2.2). The massless fermion is on shell $\left(p^{2}=0\right)$, and the nonzero gluon mass $M$ is inserted as an IR regulator, or the real gauge boson mass in electroweak processes. Performing the contour integral on $n \cdot k$, we obtain

$$
\begin{aligned}
\tilde{M}_{n} & =-\frac{\alpha_{s} C_{F}}{2 \pi} \frac{\left(\mu^{2} e^{\gamma_{\mathrm{E}}}\right)^{\epsilon} \nu^{\eta}}{\Gamma(1-\epsilon)} \int_{0}^{p_{+}} d k_{+} k_{+}^{-1-\eta}\left(1-\frac{k_{+}}{p_{+}}\right) \int_{0}^{\infty} \frac{d \mathbf{k}_{\perp}^{2}\left(\mathbf{k}_{\perp}^{2}\right)^{-\epsilon}}{\mathbf{k}_{\perp}^{2}+\frac{p_{+}-k_{+}}{p_{+}} M^{2}} \\
& =-\frac{\alpha_{s} C_{F}}{2 \pi} e^{\gamma_{\mathrm{E}} \epsilon} \Gamma(\epsilon)\left(\frac{\mu^{2}}{M^{2}}\right)^{\epsilon}\left(\frac{\nu}{p_{+}}\right)^{\eta} \int_{0}^{1} d x(1-x)^{1-\epsilon} x^{-1-\eta},
\end{aligned}
$$

where $p_{+} \equiv \bar{n} \cdot p$ is the largest component of the external momentum $p$, and $x=k_{+} / p_{+}$.

In eq. (2.9), the pole in $\eta$ comes from the region $k_{+} \rightarrow 0$. Since the momentum $k_{+}$of the collinear gluon has an upper limit $p_{+}$, there is no rapidity divergence as $k_{+} \rightarrow \infty$ in this naive collinear amplitude by itself. However, as we have explained in Introduction, it is cancelled when we subtract the zero-bin contribution because the soft sector shares the same phase space. Due to the zero-bin subtraction, the rapidity divergence in the collinear sector is pulled up to the divergence for $k_{+} \rightarrow \infty$.

The zero-bin contribution is obtained from eq. (2.9) by taking the limits $k_{+} \ll p_{+}$or $x \rightarrow 0$ in the integrand, and the upper limit in the integral of $x$ to infinity. It is given by

$$
M_{n}^{\varnothing}=-\frac{\alpha_{s} C_{F}}{2 \pi} e^{\gamma_{\mathrm{E}} \epsilon} \Gamma(\epsilon)\left(\frac{\mu^{2}}{M^{2}}\right)^{\epsilon}\left(\frac{\nu}{p_{+}}\right)^{\eta} \int_{0}^{\infty} d x x^{-1-\eta} .
$$


(a)

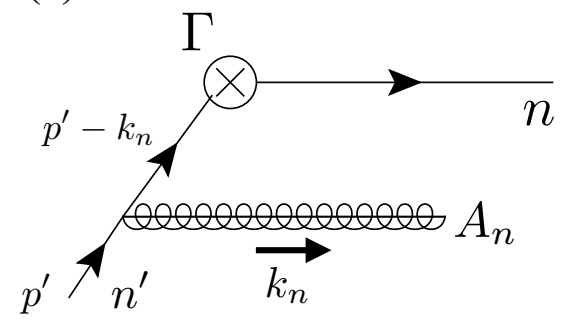

(b)

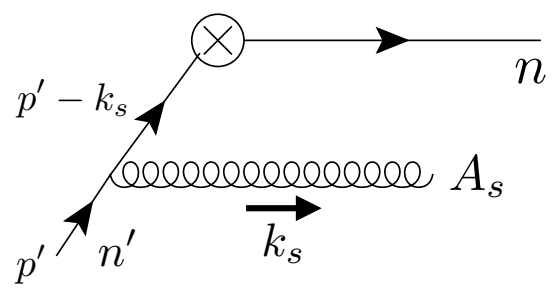

Figure 2. (a) Collinear and (b) soft gluon emissions from the $n^{\prime}$-collinear sector in the current $\bar{q}_{n} \Gamma q_{n^{\prime}}$, which yield the leading contributions to $W_{n}$ and $S_{n^{\prime}}$ respectively. If $\bar{n} \cdot k_{s} \rightarrow \infty$ and $n \cdot k_{s} \rightarrow 0$ in the soft phase space, $k_{s}$ becomes the soft version of the $n$-collinear momentum $k_{n}$, and the rapidity divergence arises when we separate the soft and collinear gluons in SCET.

Then the legitimate collinear contribution is obtained by subtracting the zero-bin contribution, eq. (2.10), from the naive collinear contribution, eq. (2.9). It is given as

$$
\begin{aligned}
M_{n} & =\tilde{M}_{n}-M_{n}^{\varnothing} \\
& =-\frac{\alpha_{s} C_{F}}{2 \pi} e^{\gamma_{\mathrm{E}} \epsilon} \Gamma(\epsilon)\left(\frac{\mu^{2}}{M^{2}}\right)^{\epsilon}\left[\int_{0}^{1} \frac{d x}{x}\left((1-x)^{1-\epsilon}-1\right)-\left(\frac{\nu}{p_{+}}\right)^{\eta} \int_{1}^{\infty} \frac{d x}{x^{1+\eta}}\right] \\
& =\frac{\alpha_{s} C_{F}}{2 \pi}\left[\left(\frac{1}{\epsilon}+\ln \frac{\mu^{2}}{M^{2}}\right)\left(\frac{1}{\eta}+\ln \frac{\nu}{\bar{n} \cdot p}+1\right)+1-\frac{\pi^{2}}{6}\right] .
\end{aligned}
$$

In the second line, we divide the integration region of the zero-bin contribution into $x \in$ $[0,1]$ and $x \in[1, \infty]$. Then the integral for $x \in[0,1]$ is combined with the naive collinear contribution $\tilde{M}_{n}$. Note that there is no divergence in the integral with $x \in[0,1]$, and we put $\eta=0$. Finally the correct rapidity divergence in the collinear sector is captured through the zero-bin subtraction.

\subsection{Rapidity regulator in the soft sector}

We have to find a consistent rapidity regulator in the soft sector that conforms to the regulator in the collinear sector. Here we extend the collinear current to $\bar{q}_{n} \Gamma q_{n^{\prime}}$, where the lightcone directions $n$ and $n^{\prime}$ are not necessarily back-to-back, but $n \cdot n^{\prime} \sim \mathcal{O}(1)$. Let us consider the configuration of figure 2 , in which a collinear or a soft gluon is emitted. In figure 2 (a), a collinear gluon from the $n^{\prime}$-collinear quark $q_{n^{\prime}}$ is emitted in the $n$ direction. It produces the collinear Wilson line $W_{n}$ at first order.

The same configuration is exhibited in figure $2(\mathrm{~b})$ where a soft gluon is emitted, producing the soft Wilson line $S_{n^{\prime}}$ at first order. The momentum of the soft gluon scales as $\left(\bar{n} \cdot k, \mathbf{k}_{\perp}, n \cdot k\right)=Q(\lambda, \lambda, \lambda)$ with a large scale $Q$. But the soft sector is an exact copy of QCD when $Q$ is taken to infinity. Therefore when the soft momentum is in the corner of phase space with $\bar{n} \cdot k \rightarrow \infty, n \cdot k \rightarrow 0$, it approaches the $n$-collinear momentum. It is the region where the rapidity divergence occurs. It means that the soft rapidity regulator associated with the $n$-collinear sector should be implemented in $S_{n^{\prime}}$, not $S_{n}$. 
The collinear rapidity regulator for $W_{n}$ in eq. (2.2) for figure 2 (a) is given by $(\nu / \bar{n} \cdot k)^{\eta}$. And the rapidity divergence shows up as poles of $1 / \eta$ with $\bar{n} \cdot k \rightarrow \infty$. It will be consistent to use the same rapidity regulator for the soft part as $(\nu / \bar{n} \cdot k)^{\eta}$. However, the form of the Wilson lines $W_{n}$ and $S_{n^{\prime}}$ is determined by the power counting of the collinear and soft momenta. The collinear momentum and the collinear gauge field scale as $Q\left(1, \lambda, \lambda^{2}\right)$, while the soft momentum and the soft gauge field scale as $Q(\lambda, \lambda, \lambda)$. In order to take a consistent rapidity regulator in the collinear and the soft sectors, we choose the configuration of figure 2 (b) and take the $n$-collinear limit.

The soft Wilson line $S_{n^{\prime}}$ is written as

$$
S_{n^{\prime}}=\sum_{\text {perm. }} \exp \left[-g \frac{1}{n^{\prime} \cdot \mathcal{P}} n^{\prime} \cdot A_{s}\right]
$$

where $n^{\prime} \cdot \mathcal{P}$ returns the incoming momentum of the soft gluon with $p^{\prime} \cdot k_{s} \sim \bar{n}^{\prime} \cdot p^{\prime} n^{\prime} \cdot k_{s}$ and $p^{\prime} \cdot A_{s} \sim \bar{n}^{\prime} \cdot p^{\prime} n^{\prime} \cdot A_{s}$ at leading order, where $p^{\prime}$ is the $n^{\prime}$-collinear momentum. When the internal particle is integrated out in figure $2(\mathrm{~b})$, we obtain $g n^{\prime} \cdot A_{s} / n^{\prime} \cdot k$. On the other hand, if we take the $n$-collinear limit of the soft momentum $k$, it becomes $k^{\mu} \approx(\bar{n} \cdot k) n^{\mu} / 2$. In this limit $n^{\prime} \cdot k=(\bar{n} \cdot k)\left(n \cdot n^{\prime}\right) / 2$. Therefore the soft rapidity regulator to capture the divergence as $\bar{n} \cdot k \rightarrow \infty$ is given by

$$
\left(\frac{\nu}{n^{\prime} \cdot k} \cdot \frac{n \cdot n^{\prime}}{2}\right)^{\eta} \underset{\bar{n} \cdot k \rightarrow \infty}{\longrightarrow}\left(\frac{\nu}{\bar{n} \cdot k}\right)^{\eta}
$$

The important point in taking this limit is to express the original regulator $(\nu / \bar{n} \cdot k)^{\eta}$ in terms of $n^{\prime} \cdot k$, with which the soft Wilson line $S_{n^{\prime}}$ is expressed. As a consequence, we suggest that the soft rapidity regulator for figure $2(\mathrm{~b})$ is given by

$$
\left(\frac{\nu}{n^{\prime} \cdot k} \frac{n^{\prime} \cdot n}{2}\right)^{\eta}
$$

because it corresponds to the regulator $(\nu / \bar{n} \cdot k)^{\eta}$ in the limit $\bar{n} \cdot k \rightarrow \infty$. Accordingly, the soft Wilson line is modified as

$$
S_{n^{\prime}}=\sum_{\text {perm. }} \exp \left[-g \frac{1}{n^{\prime} \cdot \mathcal{P}}\left(\frac{\nu}{\left|n^{\prime} \cdot \mathcal{P}\right|} \frac{n^{\prime} \cdot n}{2}\right)^{\eta} n^{\prime} \cdot A_{s}\right] .
$$

For $S_{n}$, we switch $n$ and $n^{\prime}$. These soft Wilson lines appear in the collinear current $\bar{q}_{n} \Gamma q_{n^{\prime}}$.

Consider an $N$-jet operator with one $n$-collinear operator, and the remaining $(N-1) n_{i^{-}}$ collinear operators $(i=1, \cdots, N-1)$, in which we are interested in the rapidity divergence associated with the $n$ direction. For each $n_{i}$ direction, we can modify $S_{n_{i}}$ using different rapidity regulators with $\eta_{i}$ and $\nu_{i}$ in the form

$$
\left(\frac{\nu_{i}}{\left|n_{i} \cdot \mathcal{P}\right|} \frac{n \cdot n_{i}}{2}\right)^{\eta_{i}}
$$

It properly captures the rapidity divergence in the $n$ direction when a soft gluon is radiated from the $n_{i}$-collinear sector to the $n$ direction in the limit $\bar{n} \cdot k \rightarrow \infty$. Note that each separate rapidity scale $\nu_{i}$ can be assigned to each $n_{i}$ direction, and the corresponding rapidity divergences are cancelled when the collinear and soft contributions are added. We will discuss the $N$-jet operator in more detail in section 4 . 


\section{Sudakov form factor for the back-to-back current}

\subsection{Soft one-loop contribution to the back-to-back collinear current}

We now return to the back-to-back collinear current in eq. (2.1), and consider its soft contribution at one loop, shown in figure 1(c). Due to the presence of $\tilde{S}_{n}^{\dagger}$ and $S_{\bar{n}}$ in the current, the soft contribution contains the factor

$$
\frac{1}{(n \cdot k)(\bar{n} \cdot k)} \text {. }
$$

It provides two types of rapidity divergences as $\bar{n} \cdot k \rightarrow \infty, n \cdot k \rightarrow 0$ in the $n$-collinear sector, and the rapidity divergence as $n \cdot k \rightarrow \infty, \bar{n} \cdot k \rightarrow 0$ in the $\bar{n}$-collinear sector, while $k_{L}^{2} \equiv \bar{n} \cdot k n \cdot k \sim \mathbf{k}_{\perp}^{2}$ remains fixed. The rapidity divergence is not regulated by the dimensional regularization because it appears irrespective of the UV $\left(\mathbf{k}_{\perp}^{2} \rightarrow \infty\right)$, or the IR $\left(\mathbf{k}_{\perp}^{2} \rightarrow 0\right)$ limits.

From eq. (2.15), the soft Wilson lines $\tilde{S}_{n}^{\dagger}$ and $S_{\bar{n}}$ with the rapidity regulator are written as

$$
\begin{aligned}
& \tilde{S}_{n}^{\dagger}=\sum_{\text {perm. }} \exp \left[-g n \cdot A_{s} \frac{1}{n \cdot \mathcal{P}^{\dagger}+i \varepsilon}\left(\frac{\nu_{-}}{\left|n \cdot \mathcal{P}^{\dagger}\right|}\right)^{\eta_{-}}\right], \\
& S_{\bar{n}}=\sum_{\text {perm. }} \exp \left[-g \frac{1}{\bar{n} \cdot \mathcal{P}+i \varepsilon}\left(\frac{\nu_{+}}{|\bar{n} \cdot \mathcal{P}|}\right)^{\eta_{+}} \bar{n} \cdot A_{s}\right]
\end{aligned}
$$

with $n \cdot \bar{n}=2$. The $i \varepsilon$-prescription follows from ref. [17]. We introduce the independent rapidity regulator for each collinear direction. The rapidity regulator with $\eta_{-}$in eq. (3.2) regulates the divergence in the $\bar{n}$ direction, and the one with $\eta_{+}$in eq. (3.3) in the $n$ direction, with the corresponding two rapidity scales, $\nu_{\mp}$.

The soft one-loop contribution, before the regulator is inserted, is given as

$$
\begin{aligned}
\tilde{M}_{S} & =-2 i g^{2} C_{F} \mu \frac{2 \epsilon}{\mathrm{MS}} \int \frac{d^{D} k}{(2 \pi)^{D}} \frac{1}{\left(k^{2}-M^{2}+i \varepsilon\right)\left(k_{+}-i \varepsilon\right)\left(k_{-}-i \varepsilon\right)} \\
& =-\left.2 g^{2} C_{F} \mu \frac{2 \epsilon}{\mathrm{MS}} \int \frac{d^{D-1} k}{(2 \pi)^{D-1}} \frac{1}{2 k_{0}} \frac{1}{k_{+} k_{-}}\right|_{k_{0}=\sqrt{\mathbf{k}^{2}+M^{2}}} \\
& =-4 \pi g^{2} C_{F} \mu \frac{2 \epsilon}{\mathrm{MS}} \int \frac{d^{D} k}{(2 \pi)^{D}} \frac{\delta\left(k^{2}-M^{2}\right)}{k_{+} k_{-}} \Theta\left(k_{0}\right) \\
& =-\frac{\alpha_{s} C_{F}}{2 \pi} \frac{\left(\mu^{2} e^{\gamma_{\mathrm{E}}}\right)^{\epsilon}}{\Gamma(1-\epsilon)} \int_{0}^{\infty} d k_{+} \int_{M^{2} / k_{+}}^{\infty} d k_{-} \frac{\left(k_{+} k_{-}-M^{2}\right)^{-\epsilon}}{k_{+} k_{-}},
\end{aligned}
$$

where we first perform the contour integral on $k_{0}$, with the relation

$$
\frac{1}{2 \sqrt{\mathbf{k}^{2}+M^{2}}}=\int d k_{0} \delta\left(k^{2}-M^{2}\right) \Theta\left(k_{0}\right) .
$$

The rapidity regulators do not affect the pole structures of the contour integral, and are dropped for the moment.

Now we assign the rapidity regulators according to eqs. (3.2) and (3.3). As can be seen in figure $3(\mathrm{a})$, the green regions in the phase space, where the rapidity divergences arise, 


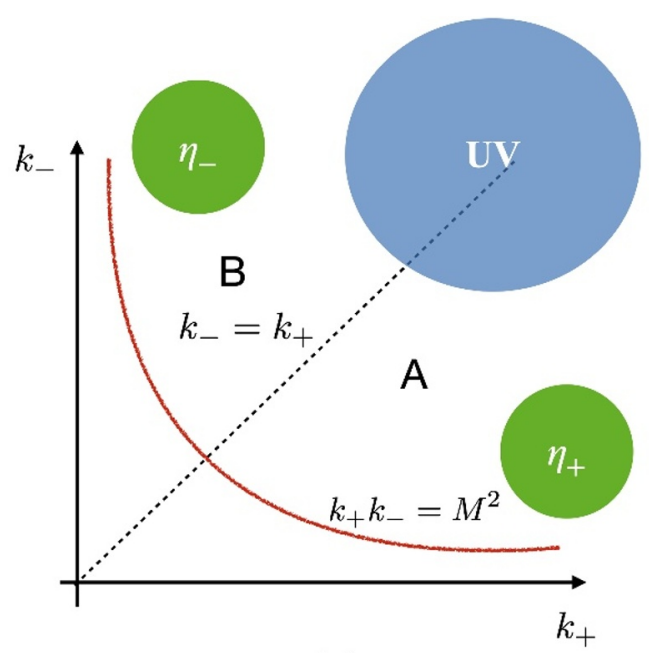

(a)

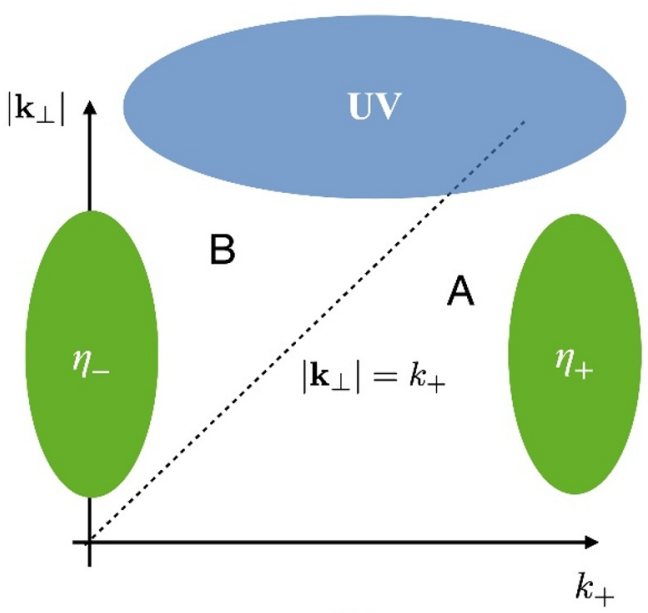

(b)

Figure 3. Structure of the phase space for the soft gluon in the back-to-back current. The rapidity divergence in the $n(\bar{n})$ direction arises in the green region with $\eta_{+}\left(\eta_{-}\right)$. (a) The phase space in the $k_{+}-k_{-}$plane with nonzero gluon mass $M$. The (red) curve $k_{+} k_{-}=M^{2}$ is the IR cutoff. (b) The phase space in $k_{+-}\left|\mathbf{k}_{\perp}\right|$ plane with massless gluons. The IR divergence arises at $\left|\mathbf{k}_{\perp}\right|=0$.

are well separated. Therefore, for practical purposes, it is convenient to divide the phase space by the line $k_{+}=k_{-}$in the $k_{+}-k_{-}$plane. Then, we can employ the regulator from $S_{\bar{n}}$ only in the region for $k_{+}>k_{-}$, while we employ the regulator from $S_{n}$ only in the region for $k_{+}<k_{-}$because the omitted regulators produce no rapidity divergence. As a result, the rapidity regulator at one loop can be written $\mathrm{as}^{2}$

$$
R_{S}\left(\nu_{+}, \nu_{-}\right)=\left(\frac{\nu_{+}}{k_{+}}\right)^{\eta_{+}} \Theta\left(k_{+}-k_{-}\right)+\left(\frac{\nu_{-}}{k_{-}}\right)^{\eta_{-}} \Theta\left(k_{-}-k_{+}\right)
$$

It amounts to specifying independent rapidity scales for different collinear directions. If we set $\eta=\eta_{+}=\eta_{-}$and $\nu=\nu_{+}=\nu_{-}$, we obtain the same result using the regulator proposed in refs. $[5,6]$, in which the soft regulator is written as

$$
R_{S}^{\prime}(\nu)=\left(\frac{\nu}{\left|k_{+}-k_{-}\right|}\right)^{\eta}
$$

As $k_{+} \rightarrow \infty, k_{-} \rightarrow 0, k_{+}$is dominant, while $k_{-}$is dominant as $k_{-} \rightarrow \infty, k_{+} \rightarrow 0$. Dividing the full phase space by the line $k_{+}=k_{-}$, the soft rapidity regulator can be written as

$$
R_{S}^{\prime}(\nu)=\left(\frac{\nu}{k_{+}}\right)^{\eta} \Theta\left(k_{+}-k_{-}\right)+\left(\frac{\nu}{k_{-}}\right)^{\eta} \Theta\left(k_{-}-k_{+}\right)
$$

which reduces to $R_{S}$ with $\nu_{+}=\nu_{-}=\nu$ at leading order, neglecting subleading corrections.

Applying eq. (3.6) to eq. (3.4), the soft contribution can be written as

$$
M_{S}=-\frac{\alpha_{s} C_{F}}{2 \pi} \frac{\left(\mu^{2} e^{\gamma_{\mathrm{E}}}\right)^{\epsilon}}{\Gamma(1-\epsilon)}\left(\nu_{+}^{\eta_{+}} \int_{A} \frac{d k_{+}}{k_{+}^{1+\eta_{+}}} \frac{d k_{-}}{k_{-}}+\nu_{-}^{\eta_{-}} \int_{B} \frac{d k_{+}}{k_{+}} \frac{d k_{-}}{k_{-}^{1+\eta_{-}}}\right)\left(k_{+} k_{-}-M^{2}\right)^{-\epsilon},
$$

\footnotetext{
${ }^{2}$ The regulator in eq. (3.6) is not new, and has been also proposed in ref. [18], where different jet vetoes along the beam directions have been considered.
} 
where the phase space $A(B)$ is the region with $k_{+}>k_{-}\left(k_{+}<k_{-}\right)$, both with $k_{+} k_{-}>M^{2}$. [See figure 3 (a).] The contribution from the region $A$ is given by

$$
\begin{aligned}
M_{S}^{A} & =-\frac{\alpha_{s} C_{F}}{2 \pi} \frac{\left(\mu^{2} e^{\gamma_{\mathrm{E}}}\right)^{\epsilon} \nu_{+}^{\eta_{+}}}{\Gamma(1-\epsilon)} \int_{M^{2}}^{\infty} \frac{d k_{L}^{2}}{k_{L}^{2}}\left(k_{L}^{2}-M^{2}\right)^{-\epsilon} \int_{k_{L}}^{\infty} \frac{d k_{+}}{k_{+}^{1+\eta_{+}}} \\
& =-\frac{\alpha_{s} C_{F}}{4 \pi}\left(\frac{\mu^{2} e^{\gamma_{\mathrm{E}}}}{M^{2}}\right)^{\epsilon}\left(\frac{\nu_{+}}{M}\right)^{\eta_{+}} \frac{\Gamma\left(\epsilon+\frac{\eta_{+}}{2}\right) \Gamma\left(\frac{\eta_{+}}{2}\right) \Gamma\left(1-\eta_{+}\right)}{\Gamma\left(1+\frac{\eta_{+}}{2}\right) \Gamma\left(1-\frac{\eta_{+}}{2}\right)} \\
& =\frac{\alpha_{s} C_{F}}{4 \pi}\left[\frac{1}{\epsilon^{2}}+\frac{1}{\epsilon} \ln \frac{\mu^{2}}{M^{2}}-\left(\frac{1}{\epsilon}+\ln \frac{\mu^{2}}{M^{2}}\right)\left(\frac{2}{\eta_{+}}+\ln \frac{\nu_{+}^{2}}{M^{2}}\right)+\frac{1}{2} \ln ^{2} \frac{\mu^{2}}{M^{2}}-\frac{\pi^{2}}{12}\right],
\end{aligned}
$$

where we require that $\eta_{+}$go to zero faster than $\epsilon^{n}$ with $n>0$. The contribution from the region $B, M_{S}^{B}$, is obtained from $M_{S}^{A}$ by switching $\left(\eta_{+}, \nu_{+}\right) \leftrightarrow\left(\eta_{-}, \nu_{-}\right)$. The complete soft contribution at one loop is given as

$$
\begin{aligned}
M_{S}=M_{S}^{A}+M_{S}^{B}= & \frac{\alpha_{s} C_{F}}{4 \pi}\left[\frac{2}{\epsilon^{2}}+\frac{2}{\epsilon} \ln \frac{\mu^{2}}{M^{2}}+\ln ^{2} \frac{\mu^{2}}{M^{2}}-\frac{\pi^{2}}{6}\right. \\
& \left.-\left(\frac{1}{\epsilon}+\ln \frac{\mu^{2}}{M^{2}}\right)\left(\frac{2}{\eta_{+}}+\ln \frac{\nu_{+}^{2}}{M^{2}}+\frac{2}{\eta_{-}}+\ln \frac{\nu_{-}^{2}}{M^{2}}\right)\right] .
\end{aligned}
$$

It is consistent with the result in ref. [6] with a single $\eta$.

\subsection{Factorization of the Sudakov form factor}

The $n$-collinear contribution at one loop in figure 1 (a) is given by eq. (2.11). Combining it with the field strength renormalization and the residue for a light quark,

$$
Z_{q}^{(1)}=-\frac{\alpha_{s} C_{F}}{4 \pi} \frac{1}{\epsilon}, \quad R_{q}^{(1)}=-\frac{\alpha_{s} C_{F}}{4 \pi}\left(\ln \frac{\mu^{2}}{M^{2}}-\frac{1}{2}\right)
$$

we obtain the complete contribution to the $n$-collinear sector at one loop as

$$
\begin{aligned}
C_{n}^{(1)} & =M_{n}+\frac{1}{2}\left(Z_{q}^{(1)}+R_{q}^{(1)}\right) \\
& =\frac{\alpha_{s} C_{F}}{4 \pi}\left[\left(\frac{1}{\epsilon}+\ln \frac{\mu^{2}}{M^{2}}\right)\left(\frac{2}{\eta_{+}}+2 \ln \frac{\nu_{+}}{p_{+}}+\frac{3}{2}\right)+\frac{9}{4}-\frac{\pi^{2}}{3}\right] .
\end{aligned}
$$

Replacing $\left(p_{+}, \eta_{+}, \nu_{+}\right)$with $\left(p_{-}^{\prime}, \eta_{-}, \nu_{-}\right)$, the bare one loop result for the $\bar{n}$-collinear sector is given by

$$
C_{\bar{n}}^{(1)}=\frac{\alpha_{s} C_{F}}{4 \pi}\left[\left(\frac{1}{\epsilon}+\ln \frac{\mu^{2}}{M^{2}}\right)\left(\frac{2}{\eta_{-}}+2 \ln \frac{\nu_{-}}{p_{-}^{\prime}}+\frac{3}{2}\right)+\frac{9}{4}-\frac{\pi^{2}}{3}\right] .
$$

From the SCET current $V_{\mathrm{SCET}}^{\mu}=F_{\mathrm{SCET}} \cdot \bar{u}_{n}(p) \gamma_{\perp}^{\mu} u_{\bar{n}}\left(p^{\prime}\right)$ in eq. (2.1), the Sudakov form factor is factorized as

$$
F_{\mathrm{SCET}}\left(Q^{2}, \mu ; M^{2}\right)=C_{n}\left(p_{+}, \mu, \nu_{+} ; M^{2}\right) C_{\bar{n}}\left(p_{-}^{\prime}, \mu, \nu_{-} ; M^{2}\right) S_{n \bar{n}}\left(\mu, \nu_{+}, \nu_{-} ; M^{2}\right)
$$


where $Q^{2}=2 p \cdot p^{\prime}=p_{+} p_{-}^{\prime}$ is the momentum transfer squared to the current. To next-toleading order (NLO) in $\alpha_{s}$, the collinear and soft functions are given by $C_{n, \bar{n}}=1+C_{n, \bar{n}}^{(1)}$ and $S_{n \bar{n}}=1+M_{S}$ after the renormalization from eqs. (3.13), (3.14), and (3.11). Each rapidity scale dependence in $C_{n}$ and $C_{\bar{n}}$ is cancelled by the soft function $S_{n \bar{n}}$. But the evolution of $\nu_{+}$from $p_{+}$to $M$ and that of $\nu_{-}$from $p_{-}^{\prime}$ to $M$ are needed to resum the large logarithms of $Q / M$. The full resummation of large logarithms with the evolution of $\nu_{ \pm}$as well as $\mu$ is thoroughly explained in appendix A.1.

The advantage of introducing multiple rapidity scales $\nu_{ \pm}$in eq. (3.15) is that we can systematically deal with the cases, in which there is a hierarchy of scales between $p_{+}$and $p_{-}^{\prime}$. For example, if $p_{+} \gg p_{-}^{\prime} \gg M$, the range of the evolution in $\nu_{-}$is smaller than the range of $\nu_{+}$. It is very interesting to consider the limit $p_{+} \gg p_{-}^{\prime} \sim M$, in which we can directly describe the soft-collinear current ${ }^{3}$ from eq. (3.15). Identifying $\nu_{-} \sim M$ in $C_{\bar{n}}$ and $S_{n \bar{n}}$ in eq. (3.15), we can combine the two functions into a new soft function to describe the soft sector. We refer to section 4 for more details.

\subsection{On-shell regularization with a massless gluon}

We can also employ pure dimensional regularization with a massless gluon, in which the $\mathrm{UV}$ and IR divergences are expressed as poles in $\epsilon_{\mathrm{UV}}$ and $\epsilon_{\mathrm{IR}}$ respectively. The UV and IR divergences are separated, and the problematic mixed divergence such as $\left(1 / \epsilon_{\mathrm{UV}}\right)$. $\left(1 / \epsilon_{\mathrm{IR}}\right)$ does not appear. In this paper, we use two ways to treat the IR divergence. Firstly, a nonzero gluon mass $M$ is introduced to regulate the IR divergence. Secondly, the IR divergence is explicitly computed and treated as a pole in $\epsilon$ using the dimensional regularization. In this subsection, we introduce the latter, which is quite nontrivial when the rapidity divergence is involved. The technical detail is instructive, but it has not received adequate attention.

Compared to eq. (2.11) with nonzero $M$, the $n$-collinear contribution with $M=0$ is given by

$$
\begin{aligned}
M_{n} & =\tilde{M}_{n}-M_{n}^{\varnothing} \\
& =-\frac{\alpha_{s} C_{F}}{2 \pi} \frac{\left(\mu^{2} e^{\gamma_{\mathrm{E}}}\right)^{\epsilon}}{\Gamma(1-\epsilon)} \int_{0}^{\infty} d \mathbf{k}_{\perp}^{2}\left(\mathbf{k}_{\perp}^{2}\right)^{-1-\epsilon}\left[\int_{0}^{1} \frac{d x}{x}(1-x)-\left(\frac{\nu_{+}}{p_{+}}\right)^{\eta_{+}} \int_{0}^{\infty} \frac{d x}{x^{1+\eta_{+}}}\right] \\
& =-\frac{\alpha_{s} C_{F}}{2 \pi}\left(\frac{1}{\epsilon_{\mathrm{UV}}}-\frac{1}{\epsilon_{\mathrm{IR}}}\right)\left[\int_{0}^{1} \frac{d x}{x}((1-x)-1)-\left(\frac{\nu_{+}}{p_{+}}\right)^{\eta_{+}} \int_{1}^{\infty} \frac{d x}{x^{1+\eta_{+}}}\right] \\
& =\frac{\alpha_{s} C_{F}}{2 \pi}\left(\frac{1}{\epsilon_{\mathrm{UV}}}-\frac{1}{\epsilon_{\mathrm{IR}}}\right)\left(\frac{1}{\eta_{+}}+\ln \frac{\nu_{+}}{p_{+}}+1\right) .
\end{aligned}
$$

Here the integration over $\mathbf{k}_{\perp}^{2}$ in pure dimensional regularization is expressed as

$$
\mu^{2 \epsilon} \int_{0}^{\infty} d \mathbf{k}_{\perp}^{2}\left(\mathbf{k}_{\perp}^{2}\right)^{-1-\epsilon}=\frac{1}{\epsilon_{\mathrm{UV}}}-\frac{1}{\epsilon_{\mathrm{IR}}} .
$$

\footnotetext{
${ }^{3} \mathrm{~A}$ similar situation has been discussed in ref. [6] in the "the lab frame". But our approach here is different. We focus on the universality and the extension of the factorization introducing multiple rapidity scales. Starting from the factorized back-to-back current, we directly describe a new soft sector through recombining one collinear sector and the soft sector, setting the relevant rapidity scales to be soft.
} 
The rapidity regulator in the integral over $x \in[0,1]$ is not necessary since there is no rapidity divergence. With the self-energy contribution where the residue is given by $R_{q}^{(1)}=$ $\alpha_{s} C_{F} /\left(4 \pi \epsilon_{\mathrm{IR}}\right)$, we obtain the $n$-collinear contribution at one loop as

$$
C_{n}^{(1)}=M_{n}+\frac{1}{2}\left(Z_{q}^{(1)}+R_{q}^{(1)}\right)=\frac{\alpha_{s} C_{F}}{4 \pi}\left(\frac{1}{\epsilon_{\mathrm{UV}}}-\frac{1}{\epsilon_{\mathrm{IR}}}\right)\left(\frac{2}{\eta_{+}}+2 \ln \frac{\nu_{+}}{p_{+}}+\frac{3}{2}\right) .
$$

Similarly, the $\bar{n}$-collinear contribution is given by

$$
C_{\bar{n}}^{(1)}=\frac{\alpha_{s} C_{F}}{4 \pi}\left(\frac{1}{\epsilon_{\mathrm{UV}}}-\frac{1}{\epsilon_{\mathrm{IR}}}\right)\left(\frac{2}{\eta_{-}}+2 \ln \frac{\nu_{-}}{p_{-}^{\prime}}+\frac{3}{2}\right) .
$$

The soft virtual contribution with a massless gluon, yet without the rapidity regulator, in figure 1 (c) is given as

$$
\begin{aligned}
\tilde{M}_{S} & =-4 \pi g^{2} C_{F} \mu_{\overline{\mathrm{MS}}}^{2 \epsilon} \int \frac{d^{D} k}{(2 \pi)^{D}} \frac{\delta\left(k^{2}\right)}{k_{+} k_{-}} \Theta\left(k_{0}\right) \\
& =-\frac{\alpha_{s} C_{F}}{2 \pi} \frac{\left(\mu^{2} e^{\gamma_{\mathrm{E}}}\right)^{\epsilon}}{\Gamma(1-\epsilon)} \int \frac{d k_{+}}{k_{+}} \frac{d k_{-}}{k_{-}} d \mathbf{k}_{\perp}^{2}\left(\mathbf{k}_{\perp}^{2}\right)^{-\epsilon} \delta\left(k^{2}\right) \Theta\left(k_{0}\right) .
\end{aligned}
$$

Applying the rapidity regulator in eq. (3.6), we divide the soft phase space into the regions $A\left(k_{+}>k_{-}\right)$and $B\left(k_{+}>k_{-}\right)$. To compute the contribution from the region $A$, it is useful to consider the phase space in $\left(k_{+},\left|\mathbf{k}_{\perp}\right|\right)$ in figure $3(\mathrm{~b})$.

The contribution from the region $A$ in figure 3 (b) can be written as

$$
\begin{aligned}
M_{S}^{A} & \equiv M_{S}^{A 1}+M_{S}^{A 2} \\
& =-\frac{\alpha_{s} C_{F}}{2 \pi} \frac{\left(\mu^{2} e^{\gamma_{\mathrm{E}}}\right)^{\epsilon} \nu_{+}^{\eta_{+}}}{\Gamma(1-\epsilon)}\left[\int_{\Lambda^{2}}^{\infty} d \mathbf{k}_{\perp}^{2}\left(\mathbf{k}_{\perp}^{2}\right)^{-1-\epsilon}+\int_{0}^{\Lambda^{2}} d \mathbf{k}_{\perp}^{2}\left(\mathbf{k}_{\perp}^{2}\right)^{-1-\epsilon}\right] \int_{\left|\mathbf{k}_{\perp}\right|}^{\infty} d k_{+} k_{+}^{-1-\eta_{+}},
\end{aligned}
$$

where we divide the integration region for $\mathbf{k}_{\perp}^{2}$ into $\left[\Lambda^{2}, \infty\right]$ and $\left[0, \Lambda^{2}\right]$ in order to separate the UV and IR divergences. The dependence on the arbitrary scale $\Lambda^{2}$ cancels at the end of calculation. The two terms in eq. (3.21) are labelled as $M_{S}^{A 1}$ and $M_{S}^{A 2}$, and are given by

$$
\begin{aligned}
& M_{S}^{A 1}=\frac{\alpha_{s} C_{F}}{4 \pi}\left[\frac{1}{\epsilon_{\mathrm{UV}}^{2}}+\frac{1}{\epsilon_{\mathrm{UV}}} \ln \frac{\mu^{2}}{\nu_{+}^{2}}-\frac{2}{\eta_{+}}\left(\frac{1}{\epsilon_{\mathrm{UV}}}+\ln \frac{\mu^{2}}{\Lambda^{2}}\right)+\frac{1}{2} \ln ^{2} \frac{\mu^{2}}{\nu_{+}^{2}}-\frac{1}{2} \ln ^{2} \frac{\nu_{+}^{2}}{\Lambda^{2}}-\frac{\pi^{2}}{12}\right], \\
& M_{S}^{A 2}=-\frac{\alpha_{s} C_{F}}{4 \pi}\left[\frac{1}{\epsilon_{\mathrm{IR}}^{2}}+\frac{1}{\epsilon_{\mathrm{IR}}} \ln \frac{\mu^{2}}{\nu_{+}^{2}}-\frac{2}{\eta_{+}}\left(\frac{1}{\epsilon_{\mathrm{IR}}}+\ln \frac{\mu^{2}}{\Lambda^{2}}\right)+\frac{1}{2} \ln ^{2} \frac{\mu^{2}}{\nu_{+}^{2}}-\frac{1}{2} \ln ^{2} \frac{\nu_{+}^{2}}{\Lambda^{2}}-\frac{\pi^{2}}{12}\right] .
\end{aligned}
$$

Combining these two contributions, we have

$$
M_{S}^{A}=\frac{\alpha_{s} C_{F}}{4 \pi}\left[\frac{1}{\epsilon_{\mathrm{UV}}^{2}}-\frac{1}{\epsilon_{\mathrm{IR}}^{2}}-\left(\frac{1}{\epsilon_{\mathrm{UV}}}-\frac{1}{\epsilon_{\mathrm{IR}}}\right)\left(\frac{2}{\eta_{+}}+\ln \frac{\nu_{+}^{2}}{\mu^{2}}\right)\right] .
$$

The contribution from the region $B$ can be obtained from eq. (3.24) by switching $\left(\eta_{+}, \nu_{+}\right) \rightarrow$ $\left(\eta_{-}, \nu_{-}\right)$. Finally the soft contribution at one loop using the pure on-shell dimensional regularization is given by

$$
M_{S}=\frac{\alpha_{s} C_{F}}{2 \pi}\left[\frac{1}{\epsilon_{\mathrm{UV}}^{2}}-\frac{1}{\epsilon_{\mathrm{IR}}^{2}}-\left(\frac{1}{\epsilon_{\mathrm{UV}}}-\frac{1}{\epsilon_{\mathrm{IR}}}\right)\left(\frac{1}{\eta_{+}}+\frac{1}{\eta_{-}}+\ln \frac{\nu_{+} \nu_{-}}{\mu^{2}}\right)\right] .
$$


The total contributions from eqs. (3.18), (3.19) and (3.25) are free of the rapidity scales and the IR divergence of the full theory is reproduced. With $Q^{2}=p_{+} p_{-}^{\prime}$, the (bare) one loop correction to the form factor in SCET is given as

$$
\begin{aligned}
F_{\mathrm{SCET}}^{(1)}\left(Q^{2}, \mu\right) & =C_{n}^{(1)}+C_{\bar{n}}^{(1)}+M_{S} \\
& =\frac{\alpha_{s} C_{F}}{2 \pi}\left[\frac{1}{\epsilon_{\mathrm{UV}}^{2}}-\frac{1}{\epsilon_{\mathrm{IR}}^{2}}+\left(\frac{1}{\epsilon_{\mathrm{UV}}}-\frac{1}{\epsilon_{\mathrm{IR}}}\right)\left(\ln \frac{\mu^{2}}{Q^{2}}+\frac{3}{2}\right)\right] .
\end{aligned}
$$

\subsection{Soft contributions to timelike processes}

So far we have considered the back-to-back collinear current with the spacelike momentum transfer. For the current with the timelike momentum transfer as in Drell-Yan (DY) process, the current in SCET is given by

$$
V_{\mathrm{DY}, \mathrm{SCET}}^{\mu}=\left\langle 0\left|\bar{\xi}_{n} W_{n} S_{n}^{\dagger} \gamma_{\perp}^{\mu} S_{\bar{n}} W_{\bar{n}}^{\dagger} \xi_{\bar{n}}\right| p p^{\prime}\right\rangle,
$$

where $p\left(p^{\prime}\right)$ is the incoming $n$ - $\left(\bar{n}\right.$-) collinear momentum. Compared to eq. $(2.1), \tilde{S}_{n}^{\dagger}$ is replaced by $S_{n}^{\dagger}[17]$. The soft Wilson line $S_{n}^{\dagger}$ from the $n$-collinear antiquark is given by

$$
S_{n}^{\dagger}=\exp \left[-g n \cdot A_{s} \frac{1}{n \cdot \mathcal{P}^{\dagger}-i \epsilon}\right]
$$

The matrix element of the full-theory current is schematically factorized as

$$
\left\langle 0\left|\bar{q}_{n} \gamma^{\mu} q_{\bar{n}}\right| p p^{\prime}\right\rangle=H_{\mathrm{DY}}\left(-Q^{2}, \mu\right) V_{\mathrm{DY}, \mathrm{SCET}}^{\mu}(\mu) \sim H_{\mathrm{DY}} \cdot C_{n} \cdot C_{\bar{n}} \cdot S_{\mathrm{DY}} \cdot \bar{v}_{n}(p) \gamma_{\perp}^{\mu} u_{\bar{n}}\left(p^{\prime}\right),
$$

where $Q^{2}=2 p \cdot p^{\prime}=p_{+} p_{-}^{\prime}$, and $u_{\bar{n}}$ and $\bar{v}_{n}$ are the spinors for the $\bar{n}$-collinear quark and $n$ collinear antiquark respectively. The hard coefficient $H_{\mathrm{DY}}$ depends on $-Q^{2}$, in contrast to $+Q^{2}$ for a spacelike process, and its anomalous dimension for $H_{\mathrm{DY}}$ at one loop is given by

$$
\gamma_{H, \mathrm{DY}}=-\frac{\alpha_{s} C_{F}}{4 \pi}\left(4 \ln \frac{\mu^{2}}{-Q^{2}}+6\right) .
$$

The minus sign in the logarithm in $\gamma_{H, \mathrm{DY}}$ also shows up in $V_{\mathrm{DY}, \mathrm{SCET}}^{\mu}$, and it appears specifically in the soft function $S_{\mathrm{DY}}$.

Figure 4 shows the different paths of the soft Wilson lines for $S_{\mathrm{DY}}$ with respect to the spacelike process, which generates the relative minus sign in the logarithm. The amplitude for $S_{\mathrm{DY}}=\left\langle 0\left|S_{n}^{\dagger} S_{\bar{n}}\right| 0\right\rangle$ at one loop is written as

$$
\tilde{M}_{S}^{\mathrm{DY}}=-2 i g^{2} C_{F} \mu \frac{2 \epsilon}{\mathrm{MS}} \int \frac{d^{D-1} k}{(2 \pi)^{D}} I_{\mathrm{DY}}(k),
$$

where $I_{\mathrm{DY}}$ is given by

$$
\begin{aligned}
I_{\mathrm{DY}}(k) & =\int_{-\infty}^{\infty} d k_{0} \frac{1}{k^{2}+i \epsilon} \frac{1}{\left(k_{+}-i \epsilon\right)\left(k_{-}+i \epsilon\right)} \\
& =\int_{-\infty}^{\infty} \frac{d k_{0}}{\left(k_{0}+|\mathbf{k}|-i \epsilon\right)\left(k_{0}-|\mathbf{k}|+i \epsilon\right)\left(k_{0}+k_{z}-i \epsilon\right)\left(k_{0}-k_{z}+i \epsilon\right)}
\end{aligned}
$$


(a)

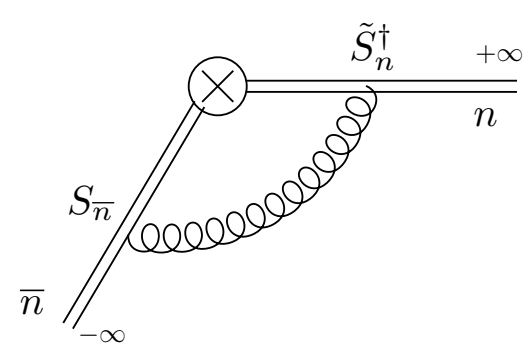

(b)

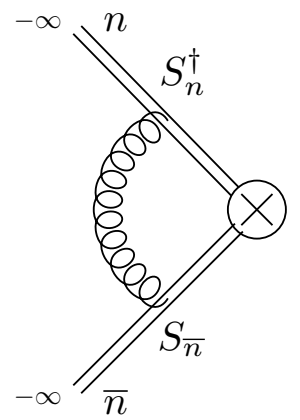

Figure 4. Soft Feynman diagrams for the virtual contributions to (a) the spacelike process and (b) the timelike (DY) process. Here the double line represents the path for a given soft Wilson line.

by setting $n^{\mu}=(1,0,0,1)$ and $\bar{n}^{\mu}=(1,0,0,-1)$, where $k_{+}=\bar{n} \cdot k=k_{0}+k_{z}$ and $k_{-}=n \cdot k=$ $k_{0}-k_{z}$. Encircling the contour in the lower-half plane, the two poles at $k_{0}=|\mathbf{k}|-i \epsilon$ and $k_{0}=k_{z}-i \epsilon$ contribute to the integral. The result can be written as $I_{\mathrm{DY}}(k)=I_{0}(k)+I_{\mathrm{T}}(k)$, where $I_{0}$ is the residue from the pole $k_{0}=|\mathbf{k}|-i \epsilon$ and $I_{\mathrm{T}}$ is from the pole $k_{0}=k_{z}-i \epsilon . I_{0}$ is given as

$$
I_{0}(k)=-\left.2 \pi i \cdot \frac{1}{2 k_{0}} \frac{1}{k_{+} k_{-}}\right|_{k_{0}=|\mathbf{k}|}=-2 \pi i \int d k_{0} \frac{\delta\left(k^{2}\right)}{k_{+} k_{-}} \Theta\left(k_{0}\right) .
$$

Note that the contribution $I_{0}(k)$ in eq. (3.31) is the same as $\tilde{M}_{S}$ in eq. (3.20) (or eq. (3.4) with $\left.M^{2}=0\right)$. So $I_{0}(k)$, or $\tilde{M}_{S}$ is common to both the spacelike and the timelike processes.

The residue $I_{\mathrm{T}}$ from the pole $k_{0}=k_{z}-i \epsilon$ is present for the timelike process only, and it is given as

$$
I_{\mathrm{T}}(k)=-2 \pi i \frac{1}{-\mathbf{k}_{\perp}^{2}+i \epsilon} \cdot \frac{1}{2 k_{z}-i \epsilon}=\frac{i \pi}{\mathbf{k}_{\perp}^{2}} \frac{1}{k_{z}-i \epsilon} .
$$

And the contribution from $I_{\mathrm{T}}$ in eq. (3.31) is given by

$$
M_{S, \mathrm{~T}}=\frac{\alpha_{s} C_{F}}{2 \pi} \frac{\left(\mu^{2} e^{\gamma_{\mathrm{E}}}\right)^{\epsilon}}{\Gamma(1-\epsilon)} \int_{0}^{\infty} d \mathbf{k}_{\perp}^{2}\left(\mathbf{k}_{\perp}^{2}\right)^{-1-\epsilon} \int_{-\infty}^{\infty} \frac{d k_{z}}{k_{z}-i \epsilon}=\frac{\alpha_{s} C_{F}}{2 \pi}(-i \pi)\left(\frac{1}{\epsilon_{\mathrm{UV}}}-\frac{1}{\epsilon_{\mathrm{IR}}}\right) .
$$

There is no rapidity divergence here, and the factor $-i \pi$ gives a negative sign in the argument of the logarithm in $M_{S}$.

As a result, applying the rapidity regulator in eq. (3.6) to $\tilde{M}_{S}^{\text {DY }}$, we obtain the soft contribution for the timelike process at one loop as

$$
\begin{aligned}
M_{S}^{\mathrm{DY}} & =M_{S}+M_{S, \mathrm{~T}} \\
& =\frac{\alpha_{s} C_{F}}{2 \pi}\left[\frac{1}{\epsilon_{\mathrm{UV}}^{2}}-\frac{1}{\epsilon_{\mathrm{IR}}^{2}}-\left(\frac{1}{\epsilon_{\mathrm{UV}}}-\frac{1}{\epsilon_{\mathrm{IR}}}\right)\left(\frac{1}{\eta_{+}}+\frac{1}{\eta_{-}}+\ln \frac{-\nu_{+} \nu_{-}}{\mu^{2}}\right)\right] .
\end{aligned}
$$

The above result can be generalized depending on the $i \varepsilon$ prescription of the soft Wilson lines. When a collinear parton in the $n_{i}$ direction is incoming and the other parton in the $n_{j}$ direction is outgoing, the sign of the term $\nu_{i} \nu_{j}$ is positive. When the two collinear partons are both incoming or outgoing, the sign is negative. 


\section{The $N$-jet operator}

The $N$-jet operator consists of $N$ collinear fields after integrating out the hard off-shell modes. It is constructed out of the collinear fermion fields $\chi_{n_{i}}=W_{n_{i}}^{\dagger} \xi_{n_{i}}$, and the collinear gauge fields $B_{n_{i} \mu}^{\perp a}=i \bar{n}_{i}^{\rho} g_{\perp}^{\mu \nu} G_{n_{i}, \rho \nu}^{b} \mathcal{W}_{n_{i}}$, where $W_{n_{i}}\left(\mathcal{W}_{n_{i}}\right)$ is the collinear Wilson line in the $n_{i}$ direction in the fundamental (adjoint) representation [3]. They are invariant under each $n_{i}$-collinear gauge transformation. For example, the 2-jet operator with quarks is $\bar{\chi}_{n_{i}} \Gamma \chi_{n_{j}}$, and the 3-jet operator is $\bar{\chi}_{n_{i}} \mathcal{B}_{n_{j} \perp}^{\mu} \Gamma_{\mu} \chi_{n_{k}}$ with some Dirac structures $\Gamma$ and $\Gamma_{\mu}$, and so on.

To be explicit, for an event with $2 N_{q}$ quark and antiquark jets, and $N_{g}$ gluon jets with $N=2 N_{q}+N_{g}$, the $N$-jet operator from the current can be schematically written as [19]

$$
J^{\mu}=C\left(p_{1}, \cdots, p_{N}\right) \prod_{i=1}^{N_{q}} \bar{\chi}_{n_{i}} \prod_{j=1}^{N_{q}} \chi_{n_{j}} \prod_{k=1}^{N_{g}} \mathcal{B}_{n_{k} \perp}^{\mu_{k}},
$$

where the color and Dirac indices are suppressed. After decoupling the soft interactions, each collinear field is redefined as

$$
\chi_{n_{i}} \rightarrow S_{n_{i}} \chi_{n_{i}}, \quad \mathcal{B}_{n_{j} \perp}^{\mu} \rightarrow \mathcal{S}_{n_{i}} \mathcal{B}_{n_{j} \perp}^{\mu}
$$

where $S_{n_{i}}\left(\mathcal{S}_{n_{i}}\right)$ is the soft Wilson line in the fundamental (adjoint) representation. Here we require that all the $N$ collinear particles form $N$ jets, implying that $n_{i} \cdot n_{j} \sim \mathcal{O}(1)$. And we consider the $N$-jet singlet operators. In $e^{+} e^{-}$annihilation, the $N$-jet operator is constructed out of the outgoing quarks and gluons, and the net color charge is zero. In hadronic collisions, we include the incoming particles to form an overall color singlet operator. For the $N$-jet operator, it involves $N$ collinear fields for the $N-2$ jets in the final state.

Let us consider the amplitude (or the amputated Green's function) by taking the matrix element of the $N$-jet operator. Employing the color-space formalism [20-22], the amplitude can be schematically written as

$$
\left|\mathcal{M}_{N}\{p\}\right\rangle=\sum_{\{a\}}\left(\left\langle\{a\} \mid \mathcal{M}_{N}\{p\}\right\rangle\right) \cdot|\{a\}\rangle
$$

where $\{p\} \equiv\left(p_{1}, p_{2}, \cdots, p_{N}\right)$ denotes the external momenta of the $N$ collinear massless partons. $|\{a\}\rangle \equiv\left|a_{1}, a_{2}, \cdots, a_{N}\right\rangle$ is the orthonormal basis in the color space, where $a_{i}$ are the color indices for the external partons. Also $\left|\mathcal{M}_{N}\{p\}\right\rangle$ can be written as

$$
\left|\mathcal{M}_{N}\{p\}\right\rangle=F_{N}(\{p\})\left|\mathcal{M}_{N}^{(0)}\{p\}\right\rangle,
$$

where $\left|\mathcal{M}_{N}^{(0)}\{p\}\right\rangle$ is the $N$-jet amplitude at tree level with the contributions from the external on-shell spinors and polarization vectors. $F_{N}(\{p\})$ is the form factor that can be expanded in powers of $\alpha_{s}$.

The form factor $F_{N}(\{p\})$ can be factorized as

$$
F_{N}(\{p\})=H_{N}\left(\left\{2 \sigma_{i j} p_{i} \cdot p_{j}\right\}, \mu\right)\left[\prod_{k}^{N} C_{k}\left(p_{k}^{+}, \mu, \nu_{k}\right)\right] S_{N}\left(\left\{\sigma_{i j} n_{i} \cdot n_{j} / 2\right\}, \mu, \nu_{1}, \cdots \nu_{N}\right),
$$




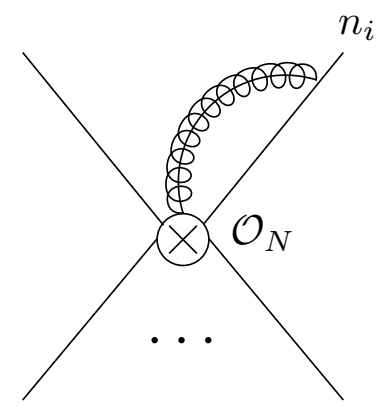

(a)

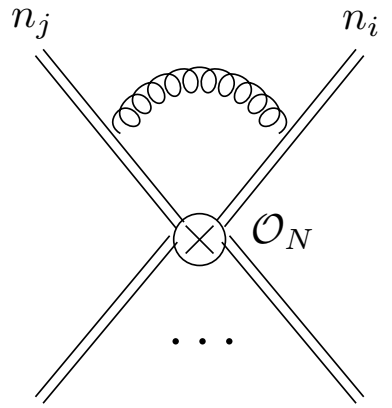

(b)

Figure 5. Feynman diagrams for the $N$-jet operator in SCET at one loop. (a) the $n_{i}$-collinear gluon exchange, (b) the soft gluon exchange between the soft Wilson lines $S_{n_{i}}$ and $S_{n_{j}}$.

where $H_{N}$ is the hard matching coefficient. The string $\left\{2 \sigma_{i j} p_{i} \cdot p_{j}\right\}$ represents all the possible combinations of the hard momentum transfers with different $i$ and $j(i, j=1, \cdots, N)$. When both $p_{i}$ and $p_{j}$ are all incoming or all outgoing, $\sigma_{i j}=-1$, and otherwise $\sigma_{i j}=+1$. The corresponding string $\left\{\sigma_{i j} n_{i} \cdot n_{j} / 2\right\}$ appears in $S_{N}$. Since all the external partons are on-shell, the momentum can be written as $p_{i}^{\mu}=p_{i}^{+} n_{i}^{\mu} / 2$ with $p_{i}^{+}=\bar{n}_{i} \cdot p_{i}$.

Let us consider the one-loop contribution to $F_{N}(\{p\})$ in SCET. The $n_{i}$-collinear contribution at one loop for figure 5 (a) can be obtained from eq. (3.19), and is given by

$$
C_{i}^{(1)}\left(p_{i}^{+}, \mu, \nu_{i}\right)=\frac{\alpha_{s}}{4 \pi}\left(\frac{1}{\epsilon_{\mathrm{UV}}}-\frac{1}{\epsilon_{\mathrm{IR}}}\right)\left[\mathbf{T}_{i}^{2}\left(\frac{2}{\eta_{i}}+2 \ln \frac{\nu_{i}}{p_{i}^{+}}\right)+\frac{\hat{\gamma}_{i}}{2}\right],
$$

where $\hat{\gamma}_{i}=3 C_{F}$ for a quark or an antiquark, and $\beta_{0}$ for a gluon. $\mathbf{T}_{i}$ is the color charge of the $i$-th collinear particle, and $\mathbf{T}_{i}^{2}=\mathbf{T}_{i}^{a} \cdot \mathbf{T}_{i}^{a}$ is $C_{F}$ for a quark or an antiquark and $C_{A}$ for a gluon.

Let us now consider the one-loop calculation for $S_{N}$. For a soft gluon exchange between $S_{n_{i}}$ and $S_{n_{j}}$ in figure $5(\mathrm{~b}),{ }^{4}$ the amplitude contains the factor

$$
\frac{1}{n_{i} \cdot k} \frac{1}{n_{j} \cdot k}
$$

The rapidity divergence can occur both in the $n_{i}$ and $n_{j}$ directions. To clarify this, we can apply the same reasoning employed in the back-to-back current. The rapidity divergence in the $n_{i}$ direction arises when $n_{i} \cdot k \rightarrow 0$ and $n_{j} \cdot k \approx\left(n_{i} \cdot n_{j} / 2\right) \bar{n}_{i} \cdot k \rightarrow \infty$, and the rapidity divergence in the $n_{j}$ direction arises when $n_{j} \cdot k \rightarrow 0$ and $n_{i} \cdot k \approx\left(n_{j} \cdot n_{i} / 2\right) \bar{n}_{j} \cdot k \rightarrow \infty$ with $\mathbf{k}_{\perp}^{2}$ fixed.

As discussed in section 2.3, we can employ the rapidity regulators in the soft Wilson lines in eq. (2.15). For the rapidity divergence in the $n_{i}$ direction, the rapidity regulator is inserted in $S_{n_{j}}$, and the regulator for the divergence in the $n_{j}$ direction, it is inserted in

\footnotetext{
${ }^{4}$ In this section, we write the soft Wilson line in the $n$ direction collectively as $S_{n}$ for $S_{n}$ or $S_{n}^{\dagger}$. It can be distinguished from the representation of the color charge operator $\mathbf{T}^{a}$ in the Wilson line.
} 
$S_{n_{i}}$. Then the contribution for the soft gluon exchange between $S_{n_{i}}$ and $S_{n_{j}}$ is given by

$$
M_{S}^{i j}=i g^{2} \mu_{\mathrm{MS}}^{2 \epsilon} \mathbf{T}_{i} \cdot \mathbf{T}_{j} \int \frac{d^{D} k}{(2 \pi)^{D}} \frac{n_{i} \cdot n_{j}}{k^{2}\left(n_{i} \cdot k\right)\left(n_{j} \cdot k\right)}\left(\frac{\nu_{i}}{n_{j} \cdot k} \frac{n_{i} \cdot n_{j}}{2}\right)^{\eta_{i}}\left(\frac{\nu_{j}}{n_{i} \cdot k} \frac{n_{i} \cdot n_{j}}{2}\right)^{\eta_{j}} .
$$

It is written to make the expression look symmetric, and the simultaneous appearance of the two regulators may look confusing. But in extracting the rapidity divergence in the $n_{i}$ direction, the $\eta_{j}$ regulator can be dropped because there is no pole in $\eta_{j}$, and vice versa.

We can directly compute eq. (4.8) by decomposing the momentum vector in the $n_{i}-n_{j}$ basis. Interestingly, there is another convenient way to recycle the result for the back-toback current. Let us boost the reference frame in order that two lightcone vectors $n_{i}$ and $n_{j}$ become back-to-back [23]. With the lightcone vectors $n_{i}^{\mu}=\left(1, \hat{n}_{i}\right)$ and $n_{j}^{\mu}=\left(1, \hat{n}_{j}\right)$, we find that the boost is obtained by the velocity $\boldsymbol{\beta}=\left(\hat{n}_{i}+\hat{n}_{j}\right) / 2$. The lightcone vectors in the boosted frame are given as

$$
\tilde{n}_{i}^{\prime \mu}=\left(\frac{1}{\gamma}, \frac{1}{2}\left(\hat{n}_{i}-\hat{n}_{j}\right)\right), \quad \tilde{n}_{j}^{\prime \mu}=\left(\frac{1}{\gamma}, \frac{1}{2}\left(\hat{n}_{j}-\hat{n}_{i}\right)\right),
$$

where the Lorentz factor $\gamma$ is given by

$$
\gamma=\gamma_{i j}=\left(\frac{n_{i} \cdot n_{j}}{2}\right)^{-1 / 2} .
$$

In eq. (4.9), the boosted lightcone vectors are not normalized, but can be normalized by rescaling $n_{i}^{\prime \mu}=\gamma \tilde{n}_{i}^{\prime \mu}, n_{j}^{\prime \mu}=\gamma \tilde{n}_{j}^{\prime \mu}$ to satisfy $n_{i}^{\prime} \cdot n_{j}^{\prime}=2$. After the boost, $n_{i} \cdot k \rightarrow \tilde{n}_{i}^{\prime} \cdot k^{\prime}=$ $n_{i}^{\prime} \cdot k^{\prime} / \gamma$, and $n_{j} \cdot k \rightarrow n_{j}^{\prime} \cdot k^{\prime} / \gamma$.

In eq. (4.8), the original amplitude without the rapidity regulators is boost invariant with $k \rightarrow k^{\prime}$ and $n_{i, j} \rightarrow n_{i, j}^{\prime}$. But the forms of the rapidity regulators are not. After the boost, the regulator transforms as

$$
\begin{aligned}
& \frac{\nu_{i}}{n_{j} \cdot k} \frac{n_{i} \cdot n_{j}}{2} \rightarrow \frac{\nu_{i}}{\tilde{n}_{j}^{\prime} \cdot k^{\prime}} \frac{\tilde{n}_{i}^{\prime} \cdot \tilde{n}_{j}^{\prime}}{2}=\frac{\nu_{i} / \gamma}{n_{j}^{\prime} \cdot k^{\prime}}=\frac{\nu_{i} / \gamma}{\bar{n}_{i}^{\prime} \cdot k^{\prime}}, \\
& \frac{\nu_{j}}{n_{i} \cdot k} \frac{n_{i} \cdot n_{j}}{2} \rightarrow \frac{\nu_{j} / \gamma}{n_{i}^{\prime} \cdot k^{\prime}} .
\end{aligned}
$$

Since $n_{i}^{\prime}$ and $n_{j}^{\prime}$ are back-to-back, we can use the regulator in eq. (3.6), and the soft rapidity regulator in the boosted frame is given by

$$
R_{S}^{i j}\left(\frac{\nu_{i}}{\gamma_{i j}}, \frac{\nu_{j}}{\gamma_{i j}}\right)=\left(\frac{\nu_{i} / \gamma_{i j}}{n_{j}^{\prime} \cdot k^{\prime}}\right)^{\eta_{i}} \theta\left(n_{j}^{\prime} \cdot k-n_{i}^{\prime} \cdot k\right)+\left(\frac{\nu_{j} / \gamma_{i j}}{n_{i}^{\prime} \cdot k^{\prime}}\right)^{\eta_{j}} \theta\left(n_{i}^{\prime} \cdot k-n_{j}^{\prime} \cdot k\right),
$$

with $n_{j}^{\prime}=\bar{n}_{i}^{\prime}$ and $n_{i}^{\prime}=\bar{n}_{j}^{\prime}$.

Therefore the soft function at one loop, eq. (4.8), can be obtained from the back-toback current by replacing $\nu_{i}$ with $\nu_{i} / \gamma_{i j}$. For given $i$ and $j$, the result is given by

$$
M_{S}^{i j}=\frac{\alpha_{s}}{4 \pi} \mathbf{T}_{i} \cdot \mathbf{T}_{j}\left[-2\left(\frac{1}{\epsilon_{\mathrm{UV}}^{2}}-\frac{1}{\epsilon_{\mathrm{IR}}^{2}}\right)+\left(\frac{1}{\epsilon_{\mathrm{UV}}}-\frac{1}{\epsilon_{\mathrm{IR}}}\right)\left(\frac{2}{\eta_{i}}+\frac{2}{\eta_{j}}-2 \ln \frac{\mu^{2}}{\sigma_{i j} \nu_{i} \nu_{j} / \gamma_{i j}^{2}}\right)\right],
$$


where $\sigma_{i j}=-1$ if both collinear partons in the $n_{i}, n_{j}$ directions are either incoming or outgoing, and $\sigma_{i j}=+1$ otherwise. The source of the negative sign is explained in section 3.4. The total contribution to $S_{N}$ at one loop is given as

$$
\begin{aligned}
S_{N}^{(1)} & =\frac{1}{2} \sum_{(i, j)}^{N} M_{S}^{i j} \\
& =\frac{\alpha_{s}}{4 \pi} \sum_{(i, j)}^{N} \mathbf{T}_{i} \cdot \mathbf{T}_{j}\left[-\left(\frac{1}{\epsilon_{\mathrm{UV}}^{2}}-\frac{1}{\epsilon_{\mathrm{IR}}^{2}}\right)+\left(\frac{1}{\epsilon_{\mathrm{UV}}}-\frac{1}{\epsilon_{\mathrm{IR}}}\right)\left(\frac{1}{\eta_{i}}+\frac{1}{\eta_{j}}-\ln \frac{\mu^{2} \gamma_{i j}^{2}}{\sigma_{i j} \nu_{i} \nu_{j}}\right)\right]
\end{aligned}
$$

where $(i, j)$ in the summation means that the case $i=j$ is excluded.

The total collinear contribution at one loop is given by

$$
\begin{aligned}
C_{N}^{(1)} & \equiv \sum_{i=1}^{N} C_{i}^{(1)}\left(p_{i}^{+}, \mu, \nu_{i}\right) \\
& =\frac{\alpha_{s}}{4 \pi}\left(\frac{1}{\epsilon_{\mathrm{UV}}}-\frac{1}{\epsilon_{\mathrm{IR}}}\right)\left[\sum_{(i, j)}^{N} \mathbf{T}_{i} \cdot \mathbf{T}_{j}\left(-\frac{1}{\eta_{i}}-\frac{1}{\eta_{j}}-\ln \frac{\nu_{i} \nu_{j}}{p_{i}^{+} p_{j}^{+}}\right)+\sum_{i}^{N} \frac{\hat{\gamma}_{i}}{2}\right] .
\end{aligned}
$$

Here the color factor is reorganized using the color singlet condition, $\sum_{i}^{N} \mathbf{T}_{i}=0$. Therefore $\mathbf{T}_{i}^{2}=-\sum_{j \neq i}^{N} \mathbf{T}_{i} \cdot \mathbf{T}_{j}$, and the sum of $\mathbf{T}_{i}^{2}$ is given as

$$
\sum_{i}^{N} \mathbf{T}_{i}^{2}=-\sum_{i}^{N} \sum_{j \neq i}^{N} \mathbf{T}_{i} \cdot \mathbf{T}_{j} \equiv-\sum_{(i, j)}^{N} \mathbf{T}_{i} \cdot \mathbf{T}_{j}
$$

The total contribution to the $N$-jet operator at order $\alpha_{s}$ is given by

$$
\begin{aligned}
C_{N}^{(1)}+S_{N}^{(1)}=\frac{\alpha_{s}}{4 \pi} & \left\{\sum_{(i, j)}^{N} \mathbf{T}_{i} \cdot \mathbf{T}_{j}\left[-\left(\frac{1}{\epsilon_{\mathrm{UV}}^{2}}-\frac{1}{\epsilon_{\mathrm{IR}}^{2}}\right)-\left(\frac{1}{\epsilon_{\mathrm{UV}}}-\frac{1}{\epsilon_{\mathrm{IR}}}\right) \ln \frac{\mu^{2}}{2 \sigma_{i j} p_{i} \cdot p_{j}}\right]\right. \\
& \left.+\sum_{i}^{N}\left(\frac{1}{\epsilon_{\mathrm{UV}}}-\frac{1}{\epsilon_{\mathrm{IR}}}\right) \frac{\hat{\gamma}_{i}}{2}\right\},
\end{aligned}
$$

where $2 p_{i} \cdot p_{j}=p_{i}^{+} p_{j}^{+} n_{i} \cdot n_{j} / 2$. The dependence on the scales $p_{i}^{+}$, and $p_{j}^{+}$in the collinear function and the directions $n_{i} \cdot n_{j}=2 / \gamma_{i j}^{2}$ in the soft function are combined to yield the relativistic invariant $p_{i} \cdot p_{j}$ in the final result. Note that all the dependence on the rapidity scales cancels when the collinear and the soft functions are added. Also, from eq. (4.18), it is straightforward to obtain the leading anomalous dimension of the hard part $H_{N}$ in the $N$ jet operator, using the fact that $F_{N}$ in the full theory is independent of the renormalization scale. It is given by

$$
\gamma_{H}^{N}=\frac{\alpha_{s}}{4 \pi}\left\{\sum_{(i, j)}^{N} \mathbf{T}_{i} \cdot \mathbf{T}_{j}\left(2 \ln \frac{\mu^{2}}{2 \sigma_{i j} p_{i} \cdot p_{j}}\right)-\sum_{i}^{N} \hat{\gamma}_{i}\right\},
$$

and is the same as the results in refs. [24, 25]. 
(a)

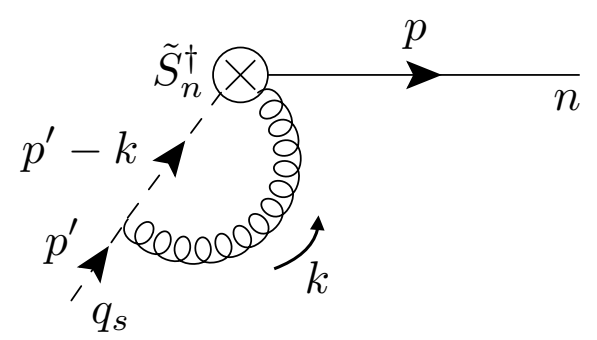

(b)

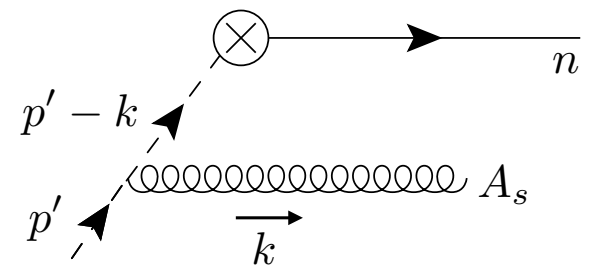

Figure 6. (a) Feynman diagram of a soft gluon exchange from a soft quark, (b) In the limit when the momentum of the soft gluon from the soft quark becomes collinear to the $n$ direction, the rapidity divergence arises.

\section{Sudakov form factor from the soft-collinear current}

Let us consider the matrix element of the soft-collinear current in SCET

$$
\left\langle p\left|J^{\mu}\right| p^{\prime}\right\rangle=H\left(Q^{2}, \mu\right)\left\langle p\left|\bar{\xi}_{n} W_{n} \gamma^{\mu} \tilde{S}_{n}^{\dagger} q_{s}\right| p^{\prime}\right\rangle
$$

where $q_{s}$ is the soft quark with momentum $p^{\prime}$. The collinear momentum $p$ and the soft momentum $p^{\prime}$ scale as $p=\left(p_{+}, p_{\perp}, p_{-}\right)=p_{+}\left(1, \lambda, \lambda^{2}\right)$ and $p^{\prime}=p_{+}(\lambda, \lambda, \lambda)$. The hard function $H\left(Q^{2}, \mu\right)$ to one loop is given as

$$
H\left(Q^{2}, \mu\right)=1+\frac{\alpha_{s} C_{F}}{4 \pi}\left(-3 \ln \frac{\mu^{2}}{Q^{2}}-\ln ^{2} \frac{\mu^{2}}{Q^{2}}-8+\frac{\pi^{2}}{6}\right),
$$

where $Q^{2}=2 p \cdot p^{\prime}$. Here the hard function can be directly computed in $\mathrm{SCET}_{\mathrm{I}}$ with the hard-collinear mode, which scales as $p_{h c}=\left(p_{h c}^{+}, p_{h c}^{\perp}, p_{h c}^{-}\right)=p_{+}\left(1, \lambda^{1 / 2}, \lambda\right)$. This mode can interact with the soft quark. After integrating out the hard-collinear mode, we can obtain $H\left(Q^{2}, \mu\right)$ with $Q^{2} \approx p_{+} p_{-}^{\prime}$. We refer to ref. [26] for the calculation of $H\left(Q^{2}, \mu\right)$ in $\operatorname{SCET}_{\mathrm{I}}$.

For the current in eq. (5.1), the Sudakov form factor $F\left(Q^{2}\right)$ can be written in a factorized form as

$$
F\left(Q^{2}\right)=H\left(Q^{2}, \mu\right) C_{n}\left(p_{+}, \mu, \nu_{+}\right) S_{q}\left(p^{\prime}, \mu, \nu_{+}\right),
$$

where $C_{n}$ and $S_{q}$ are the collinear and the soft functions for the soft-collinear current. The one-loop result for $C_{n}$ is given in eq. (3.13) or (3.18).

Let us consider the rapidity divergence in $S_{q}$ including a soft quark [figure 6 (a)]. It arises when a soft gluon from the soft quark becomes $n$-collinear and its momentum $k$ reaches $\bar{n} \cdot k \rightarrow \infty$ (and $n \cdot k \rightarrow 0$ ), as shown in figure $6(\mathrm{~b})$. (See figure 2 for comparison.) In order to be consistent with the $n$-collinear sector, the rapidity regulator should be $\left(\nu_{+} / \bar{n} \cdot k\right)^{\eta_{+}}$as $\bar{n} \cdot k$ goes to infinity. On the other hand, since the propagator in figure $6(\mathrm{~b})$ is proportional to $1 / p^{\prime} \cdot k$, the rapidity regulator in the form $\nu_{+} /\left(p^{\prime} \cdot k\right)$ is desired.

As a result, we suggest the rapidity regulator for the soft quark sector at one loop as

$$
R_{S_{q}}\left(\nu_{+} ; n^{\prime} \cdot k\right)=\left(\frac{\nu_{+}}{p^{\prime} \cdot k} \frac{n \cdot p^{\prime}}{2}\right)^{\eta_{+}}=\left(\frac{\nu_{+}}{n^{\prime} \cdot k} \frac{n \cdot n^{\prime}}{2}\right)^{\eta_{+}} \underset{\bar{n} \cdot \overrightarrow{k \rightarrow \infty}}{\longrightarrow}\left(\frac{\nu_{+}}{\bar{n} \cdot k}\right)^{\eta_{+}},
$$


where $p^{\prime \mu}=E n^{\prime \mu}, E$ is the energy of the soft quark and $n^{\prime \mu}$ is the lightcone vector for the soft massless quark. The last limit shows the consistency that we pick up the same rapidity regulator in the soft quark sector as in the $n$-collinear sector when $\bar{n} \cdot k \rightarrow \infty$.

At higher orders, it is complicated to set up a consistent rapidity regulator in the soft quark sector especially with the $n$-collinear regulator in eq. (2.2). That is because multiple soft gluon radiations from the soft quark are not eikonalized. However, as discussed in section 2.3, the origin of the rapidity divergence from the collinear and soft gluon radiations is the same. Therefore, once we set up the $n$-collinear rapidity regulator by modifying $W_{n}$ or the phase space, we can trace the corresponding factor in the multiple soft gluon radiations from the soft quark.

Before employing the regulator, the contribution to $S_{q}$ in figure 6 (a) with the pure on-shell dimensional regularization at one loop can be written as

$$
\begin{aligned}
& \tilde{M}_{S}^{q}=2 i g^{2} C_{F} \mu \frac{2 \epsilon}{\mathrm{MS}} \int \frac{d^{D} k}{(2 \pi)^{D}} \frac{n \cdot\left(p^{\prime}-k\right)}{\left(k^{2}+i \varepsilon\right)\left(k^{2}-2 k \cdot p^{\prime}+i \varepsilon\right)(n \cdot k-i \varepsilon)} \\
&=2 i g^{2} C_{F} \mu \frac{2 \epsilon}{\mathrm{MS}}\left[\frac{n \cdot p^{\prime}}{n \cdot n^{\prime}} \frac{1}{8 \pi^{2}} \frac{i(4 \pi)^{\epsilon}}{\Gamma(1-\epsilon)} \int_{0}^{\infty} d n^{\prime} \cdot k \int_{0}^{\infty} \frac{d \mathbf{k}_{\perp}^{2}\left(\mathbf{k}_{\perp}^{2}\right)^{-\epsilon}}{\mathbf{k}_{\perp}^{2}\left(\mathbf{k}_{\perp}^{2}+2 E n^{\prime} \cdot k\right)}\right. \\
&\left.\quad-\int \frac{d^{D} k}{(2 \pi)^{D}} \frac{1}{k^{2}\left(k^{2}-2 k \cdot p^{\prime}\right)}\right] .
\end{aligned}
$$

Here the momentum $k^{\mu}$ is decomposed as

$$
k^{\mu}=\frac{n^{\prime} \cdot k}{n \cdot n^{\prime}} n^{\mu}+\frac{n \cdot k}{n \cdot n^{\prime}} n^{\prime \mu}+k_{\perp}^{\mu}
$$

where $k_{\perp}^{\mu}$ is perpendicular to $n^{\mu}$ and $n^{\prime \mu}$. And $k^{2}$ is given by $k^{2}=2 n \cdot k n^{\prime} \cdot k / n \cdot n^{\prime}-\mathbf{k}_{\perp}^{2}$. In obtaining the first term in eq. (5.5), the integration measure is written as

$$
\frac{d^{D} k}{(2 \pi)^{D}}=\frac{1}{16 \pi^{3}} \frac{1}{n \cdot n^{\prime}} \frac{(4 \pi)^{\epsilon}}{\Gamma(1-\epsilon)} d n \cdot k d n^{\prime} \cdot k d \mathbf{k}_{\perp}^{2}\left(\mathbf{k}_{\perp}^{2}\right)^{-\epsilon}
$$

and the contour integral in the complex $n \cdot k$ plane is performed.

In eq. (5.5), the rapidity divergence appears only in the first term. Applying the rapidity regulator, it can be written as

$$
M_{S 1}^{q}=\frac{\alpha_{s} C_{F}}{2 \pi} \frac{\left(\mu^{2} e^{\gamma_{\mathrm{E}}}\right)^{\epsilon}}{\Gamma(1-\epsilon)} \int_{0}^{\infty} \frac{d n^{\prime} \cdot k}{n^{\prime} \cdot k} R_{S_{q}}\left(\nu_{+} ; n^{\prime} \cdot k\right) \int_{0}^{\infty} d \mathbf{k}_{\perp}^{2}\left(\mathbf{k}_{\perp}^{2}\right)^{-\epsilon}\left(\frac{1}{\mathbf{k}_{\perp}^{2}+2 E n^{\prime} \cdot k}-\frac{1}{\mathbf{k}_{\perp}^{2}}\right) .
$$

In order to separate different types of divergences clearly, the integration region for $n^{\prime} \cdot k$ is divided into $0<n^{\prime} \cdot k<\Delta$ and $n^{\prime} \cdot k>\Delta$, where $\Delta$ is an arbitary soft energy scale. Then eq. (5.8) can be written into the three parts as

$$
\begin{aligned}
& M_{S 1 a}^{q}=\frac{\alpha_{s} C_{F}}{2 \pi} \frac{\left(\mu^{2} e^{\gamma_{\mathrm{E}}}\right)^{\epsilon}}{\Gamma(1-\epsilon)} \int_{0}^{\Delta} \frac{d n^{\prime} \cdot k}{n^{\prime} \cdot k} \int_{0}^{\infty} d \mathbf{k}_{\perp}^{2}\left(\mathbf{k}_{\perp}^{2}\right)^{-\epsilon}\left(\frac{1}{\mathbf{k}_{\perp}^{2}+2 E n^{\prime} \cdot k}-\frac{1}{\mathbf{k}_{\perp}^{2}}\right) \\
& M_{S 1 b}^{q}=\frac{\alpha_{s} C_{F}}{2 \pi} \frac{\left(\mu^{2} e^{\gamma_{\mathrm{E}}}\right)^{\epsilon}}{\Gamma(1-\epsilon)} \int_{\Delta}^{\infty} \frac{d n^{\prime} \cdot k}{n^{\prime} \cdot k} \int_{0}^{\infty} \frac{d \mathbf{k}_{\perp}^{2}\left(\mathbf{k}_{\perp}^{2}\right)^{-\epsilon}}{\mathbf{k}_{\perp}^{2}+2 E n^{\prime} \cdot k} \\
& M_{S 1 c}^{q}=-\frac{\alpha_{s} C_{F}}{2 \pi} \frac{\left(\mu^{2} e^{\gamma_{\mathrm{E}}}\right)^{\epsilon}}{\Gamma(1-\epsilon)}\left(\nu_{+} \frac{n \cdot n^{\prime}}{2}\right)^{\eta_{+}} \int_{\Delta}^{\infty} \frac{d n^{\prime} \cdot k}{\left(n^{\prime} \cdot k\right)^{1+\eta_{+}}} \int_{0}^{\infty} d \mathbf{k}_{\perp}^{2}\left(\mathbf{k}_{\perp}^{2}\right)^{-1-\epsilon}
\end{aligned}
$$


The rapidity divergence arises only in eq. (5.11), and the rapidity regulator is inserted here. In eqs. (5.9) and eq. (5.10), they have IR and UV divergences respectively, and there is no need for the rapidity regulator.

Combining all the results, we obtain

$$
\begin{aligned}
M_{S 1}^{q} & =M_{S 1 a}^{q}+M_{S 1 b}^{q}+M_{S 1 c}^{q} \\
& =\frac{\alpha_{s} C_{F}}{2 \pi}\left[\frac{1}{\epsilon_{\mathrm{UV}}^{2}}-\frac{1}{\epsilon_{\mathrm{IR}}^{2}}+\left(\frac{1}{\epsilon_{\mathrm{UV}}}-\frac{1}{\epsilon_{\mathrm{IR}}}\right)\left(-\frac{1}{\eta_{+}}+\ln \frac{\mu}{n \cdot p^{\prime}}+\ln \frac{\mu}{\nu_{+}}\right)\right] .
\end{aligned}
$$

The last term in eq. (5.5) is given by

$$
M_{S 2}^{q}=\frac{\alpha_{s} C_{F}}{2 \pi}\left(\frac{1}{\epsilon_{\mathrm{UV}}}-\frac{1}{\epsilon_{\mathrm{IR}}}\right)
$$

and the self-energy contribution for the soft quark are given by

$$
Z_{q}^{(1)}+R_{q}^{(1)}=-\frac{\alpha_{s} C_{F}}{4 \pi}\left(\frac{1}{\epsilon_{\mathrm{UV}}}-\frac{1}{\epsilon_{\mathrm{IR}}}\right) .
$$

Therefore the total soft contribution to $S_{q}$ at one loop is given as

$$
\begin{aligned}
S_{q}^{(1)}\left(n \cdot p^{\prime}, \mu, \nu_{+}\right) & =M_{S 1}^{q}+M_{S 2}^{q}+\frac{1}{2}\left(Z_{q}^{(1)}+R_{q}^{(1)}\right) \\
& =\frac{\alpha_{s} C_{F}}{2 \pi}\left[\frac{1}{\epsilon_{\mathrm{UV}}^{2}}-\frac{1}{\epsilon_{\mathrm{IR}}^{2}}+\left(\frac{1}{\epsilon_{\mathrm{UV}}}-\frac{1}{\epsilon_{\mathrm{IR}}}\right)\left(-\frac{1}{\eta_{+}}+\ln \frac{\mu^{2}}{\nu_{+} n \cdot p^{\prime}}+\frac{3}{4}\right)\right] .
\end{aligned}
$$

Interestingly, note that this result can be directly obtained from the factorized result of the back-to-back current in eq. (3.15). Since the Sudakov form factor is invariant under boost, it should be the same either for the back-to-back current or for the soft-collinear current. If we keep the $n$-collinear part in common in both cases, the soft function in the soft-collinear current should be obtained as a product of the $\bar{n}$-collinear and the soft functions in the back-to-back current. It is expressed, with soft $n \cdot p^{\prime}$, as

$$
S_{q}\left(n \cdot p^{\prime}, \mu, \nu_{+}\right)=C_{\bar{n}}\left(n \cdot p^{\prime}, \mu, \nu_{-}\right) S_{n \bar{n}}\left(\mu, \nu_{+}, \nu_{-}\right) .
$$

In the back-to-back current, and in the $N$-jet operator, each factorized function has its own rapidity scale. Going further, if two factorized functions have the same rapidity scale, they can be combined, and can be regarded as a single function. From this point of view, the soft function in the soft-collinear current can be acquired from the back-to-back current. The rapidity scale $\nu_{-}$can be fixed near $n \cdot p^{\prime}$ and no running is necessary in the combination. Finally the dependence on $\nu_{-}$cancels when $C_{\bar{n}}$ and $S_{n \bar{n}}$ are combined. At one loop we easily reproduce eq. (5.15) combining the results of $C_{\bar{n}}$ and $S_{n \bar{n}}$ in eqs. (3.19) and (3.25) respectively. It is one of the advantages in using multiple rapidity scales.

The Sudakov form factor is derived from the back-to-back current and from the softcollinear current, and it turns out to be the same. It is obvious because the Sudakov form factor is a Lorentz invariant, and should be the same in all reference frames. Furthermore, since the Sudakov form factor is a physical quantity, it should be independent of the 
factorization scales both in $\mu$ and $\nu$. The main reason why we endeavor to consider the Sudakov form factor in the two cases is that the evolution with respect to the rapidity scale looks seemingly different. In the back-to-back current, there are two collinear directions involved and we introduce two rapidity scales with which each collinear part evolves using the RG equation. On the other hand, in the soft-collinear current, there is only one rapidity scale associated with a single collinear direction. However, the evolution of the Sudakov form factor is the same, and independent of the factorization scales. It is also independent of the order of evolution with respect to the renormalization scale and the rapidity scale. Because it is technical, the detail is deferred to appendix A.

\section{Sudakov form factor involving heavy quarks}

We extend our discussion on the rapidity divergence from the massless case to the massive case. When a heavy quark is boosted and its energy is much larger than the mass $m$, the rapidity divergence also occurs in the collinear corrections. Like the massless case, the origin of the rapidity divergence is the radiation of the soft gluon, which cannot access the large rapidity region, hence it gives the divergence. Therefore the rapidity divergence does not come from the naive collinear corrections to the boosted heavy quark sector, but from the zero-bin contribution.

Let us consider the collinear loop correction of figure 1 (a) for a boosted heavy quark. The naive collinear contribution is given by

$$
\tilde{M}_{n}^{m}=-\frac{\alpha_{s} C_{F}}{2 \pi} \frac{\left(\mu^{2} e^{\gamma_{\mathrm{E}}}\right)^{\epsilon}}{\Gamma(1-\epsilon)} \int_{0}^{1} d x \frac{1-x}{x} \int_{0}^{\infty} d \mathbf{k}_{\perp}^{2} \frac{\left(\mathbf{k}_{\perp}^{2}\right)^{-\epsilon}}{\mathbf{k}_{\perp}^{2}+x^{2} m^{2}}
$$

Here the collinear divergence is absent due to the heavy quark mass, but the soft divergence remains as $x \rightarrow 0$. In the massless case, the $\mathbf{k}_{\perp}^{2}$-integral is independent of $x$, and we need a rapidity regulator to regulate the region near $x=0$ (though it is not the true rapidity divergence, which is obtained after the zero-bin subtraction). Even when we include the gluon mass $M$, the denominator becomes $\mathbf{k}_{\perp}^{2}+(1-x) M^{2}$ and the same argument holds. [See eq. (2.9).] However, in eq. (6.1), the $\mathbf{k}_{\perp}^{2}$-integration affects the $x$ integral and there is no need to introduce the rapidity regulator. Because of this, one may be tempted to say that there is no rapidity divergence in the collinear part, but the true collinear contribution includes the zero-bin subtraction, from which the rapidity divergence arises.

As an illustration, let us consider the Sudakov form factor with a boosted heavy quark, in which the momentum transfer $Q$ is much larger than the quark mass $m$. The heavy quark momentum, collinear to the $n$-direction, scales as $p^{\mu}=\left(p_{+}, p_{\perp}, p_{-}\right) \sim Q\left(1, \lambda, \lambda^{2}\right)$, where $\lambda \sim m / Q$. Now we perform the soft-collinear factorization with the soft mode scaling as $p_{s}^{\mu}=\left(p_{s}^{+}, p_{s}^{\perp}, p_{s}^{-}\right) \sim Q(\lambda, \lambda, \lambda)$. The decoupling of the soft gluons from the heavy quark sector yields the same Wilson line as in the case of a light quark. Therefore the zero-bin contribution to eq. (6.1) is also the same. As a result, the true collinear one-loop 
contribution with the zero-bin subtraction is given as

$$
\begin{aligned}
M_{n}^{m}=\tilde{M}_{n}^{m}-M_{n}^{\varnothing}= & \frac{\alpha_{s} C_{F}}{2 \pi}\left[\frac{1}{\epsilon_{\mathrm{UV}}}+\ln \frac{\mu^{2}}{m^{2}}+\frac{1}{2 \epsilon_{\mathrm{IR}}}\left(\frac{1}{\epsilon_{\mathrm{IR}}}+\ln \frac{\mu^{2}}{m^{2}}\right)\right. \\
& \left.+\left(\frac{1}{\eta_{+}}+\ln \frac{\nu_{+}}{p_{+}}\right)\left(\frac{1}{\epsilon_{\mathrm{UV}}}-\frac{1}{\epsilon_{\mathrm{IR}}}\right)+\frac{1}{4} \ln ^{2} \frac{\mu^{2}}{m^{2}}+2+\frac{\pi^{2}}{24}\right] .
\end{aligned}
$$

Here the zero-bin contribution $M_{n}^{\varnothing}$ is the same as eq. (3.16), and the details of the calculation for eq. (6.2) are presented in appendix B.1. Note that the collinear part for the boosted heavy quark has the same rapidity divergence as that of the light quark. This is not surprising because the rapidity divergence comes from the soft dynamics, i.e., the zero-bin contribution as far as the collinear sector is concerned.

\subsection{Heavy-to-heavy form factor}

Let us consider the situation in which the incoming and outgoing heavy quarks have large energy $\sim Q$ and they are moving in the opposite direction. In this case, the description of the form factor becomes more intriguing because the heavy quark mass $m$ enters into the system. Because the transferred momentum $Q$ is much larger than the quark mass $m$, the form factor involves a large logarithm of $Q / \mathrm{m}$. So far, the resummation of this large logarithm has been considered in full QCD [27-29], that is based on solving the so-called "KG integro-differential equation" [30,31]. In SCET the large logarithm of $Q / m$ can be fully resummed by the evolution with respect to the rapidity scale, in conjunction with the evolution with respect to the renormalization scale.

After integrating out hard interactions, the heavy-to-heavy form factor is matched onto SCET as

$$
F\left(Q^{2}, m^{2}\right)=H\left(Q^{2}, \mu\right) F_{\mathrm{SCET}}\left(Q^{2}, m^{2}, \mu\right) .
$$

When $Q \gg m$, the mass dependence in hard interactions can be ignored. Therefore the hard function $H$ in eq. (6.3) is the same as that of the light quark and the one-loop result is given by eq. (5.2). Since the form factor is scale invariant, it can be computed at any scale. However, the structure of the complete factorization depends on which scale $\mu$ for $F_{\text {SCET }}$ we consider.

\subsubsection{Factorization with $Q \gg m \sim \mu$}

When $Q \gg m \sim \mu$, the form factor in SCET, $F_{\mathrm{SCET}}$, can be factorized into the $n$ - and $\bar{n}$-collinear parts and the soft part. The boosted heavy quark sectors are described by the $n$ - and $\bar{n}$-collinear interactions respectively, where the $n$ - and $\bar{n}$-collinear momenta scale as $p_{n}^{\mu}=\left(\bar{n} \cdot p, p_{\perp}, n \cdot p\right) \sim Q\left(1, \lambda, \lambda^{2}\right)$ and $p_{\bar{n}}^{\mu}=Q\left(\lambda^{2}, \lambda, 1\right)$ with $\lambda \sim m / Q$. The collinear interactions are legitimately described by the massive version of SCET (SCET $\mathrm{M}$ ) [32-34]. The soft interactions with the momentum scaling $p_{s}^{\mu} \sim Q(\lambda, \lambda, \lambda)$ mediates the crosstalk between the two heavy, collinear sectors. As explained above, the soft part remains unchanged, compared to the case of the light quark. Therefore, $F_{\mathrm{SCET}}$ is written as

$$
F_{\mathrm{SCET}}\left(Q^{2}, m^{2}, \mu\right)=C_{m, n}\left(p_{+}, m^{2}, \mu, \nu_{+}\right) C_{m, \bar{n}}\left(p_{-}^{\prime}, m^{2}, \mu, \nu_{-}\right) S_{n \bar{n}}\left(\mu, \nu_{+}, \nu_{-}\right),
$$


where the largest external momentum component in the $n(\bar{n})$-direction is given by $p_{+}$ $\left(p_{-}^{\prime}\right)$. The soft function $S_{n \bar{n}}$ appears in eq. (3.15) with the non-zero gluon mass $M$. Here we use the pure dimensional regularization by expressing the IR divergence as poles in $\epsilon_{\mathrm{IR}}$. The bare one-loop result for $S_{n \bar{n}}$ is given in eq. (3.25).

The complete one-loop result for the collinear part $C_{n}$ can be obtained by the sum of $M_{n}^{m}$ in eq. (6.2) and the contribution of the self energy diagram as

$$
C_{m, n}^{(1)}=M_{n}^{m}+\frac{1}{2}\left(Z_{Q}^{(1)}+R_{Q}^{(1)}\right)
$$

Here the wavefunction renormalization and the residue of the heavy quark are given by

$$
Z_{Q}^{(1)}=-\frac{\alpha_{s} C_{F}}{4 \pi} \frac{1}{\epsilon_{\mathrm{UV}}}, \quad R_{Q}^{(1)}=-\frac{\alpha_{s} C_{F}}{4 \pi}\left(\frac{2}{\epsilon_{\mathrm{IR}}}+3 \ln \frac{\mu^{2}}{m^{2}}+4\right) .
$$

The bare one-loop result, $C_{m, n}^{(1)}$, is given as

$$
\begin{aligned}
C_{m, n}^{(1)}\left(p_{+}, \mu, \nu_{+}, m^{2}\right)= & \frac{\alpha_{s} C_{F}}{4 \pi}\left[\frac{3}{2} \frac{1}{\epsilon_{\mathrm{UV}}}+\frac{1}{\epsilon_{\mathrm{IR}}^{2}}+\frac{1}{\epsilon_{\mathrm{IR}}}\left(-1+\ln \frac{\mu^{2}}{m^{2}}\right)\right. \\
& \left.+2\left(\frac{1}{\epsilon_{\mathrm{UV}}}-\frac{1}{\epsilon_{\mathrm{UV}}}\right)\left(\frac{1}{\eta_{+}}+\ln \frac{\nu_{+}}{p_{+}}\right)+\frac{1}{2} \ln ^{2} \frac{\mu^{2}}{m^{2}}+\frac{1}{2} \ln \frac{\mu^{2}}{m^{2}}+2+\frac{\pi^{2}}{12}\right],
\end{aligned}
$$

and $C_{m, \bar{n}}^{(1)}$ can be obtained from eq. (6.7) by replacing $\left\{p_{+}, \eta_{+}, \nu_{+}\right\}$with $\left\{p_{-}^{\prime}, \eta_{-}, \nu_{-}\right\}$.

The heavy quark mass in the collinear parts regularizes the collinear divergence, but it does not affect the renormalization behavior with respect to the scales $\mu$ and $\nu$. Hence the anomalous dimensions for $\mu$ and $\nu$ in each factorized function of eq. (6.4) are the same as those in the case of the light quark. Also the exponentiated evolution kernel to resum large logarithms is given by the same form as the form factor with light quarks. The resummed result for the light form factor is given in eqs. (A.34) and (A.38). In the exponentiation, The newly added term by applying the $\nu$-evolution is given by

$$
-\ln \frac{\nu_{n}^{+} \nu_{\bar{n}}^{-}}{\nu_{s}^{+} \nu_{s}^{-}} \cdot a\left[\Gamma_{C}\right]\left(\mu_{s}, M\right) .
$$

For the heavy-to-heavy form factor, the soft scales are given by $\nu_{s}^{ \pm} \sim \mu_{s} \sim m$. The gluon mass $M$ to regularize the IR divergence also scales as $m$. Therefore, to NLL accuracy, eq. (6.8) is given as

$$
-\ln \frac{Q^{2}}{m^{2}} \cdot a\left[\Gamma_{C}\right](m, M) \approx-\frac{\alpha_{s}(m) C_{F}}{2 \pi} \ln \frac{Q^{2}}{m^{2}} \ln \frac{m^{2}}{M^{2}} .
$$

\subsubsection{Factorization with $Q \gg m \gg \mu$}

When $\mu$ is much smaller than the heavy quark mass $m$, the heavy quark mass $m$ and the collinear interactions with offshellnesses $p_{n}^{2} \sim p_{\bar{n}}^{2} \sim m^{2}$ are integrated out. Then the heavy 
quarks undergo the collinear-soft (csoft) interactions [35]. The csoft momenta $p_{c s}\left(p_{\overline{c s}}\right)$ in the $n(\bar{n})$ direction scale as

$$
p_{c s}^{\mu}=\left(p_{c s}^{+}, p_{c s}^{\perp}, p_{c s}^{-}\right)=Q \zeta\left(1, \lambda, \lambda^{2}\right), \quad p_{c s}^{\mu}=Q \zeta\left(\lambda^{2}, \lambda, 1\right)
$$

where $\zeta$ is an another small parameter of order $\mu / m$.

For the heavy quark with the csoft interaction, its momentum can be expressed with a fixed velocity $v$ as

$$
p^{\mu}=m v^{\mu}+k^{\mu},
$$

with $v^{2}=1$. For the outgoing heavy quark moving in the $n$ direction, the velocity $v_{n}^{\mu}$ scales as $\left(v_{n}^{+}, v_{n}^{\perp}, v_{n}^{-}\right)=(1 / \lambda, 1, \lambda)$. For the incoming quark in the $\bar{n}$ direction, the velocity scales as $v_{\bar{n}}^{\mu}=(\lambda, 1,1 / \lambda)$. These heavy quarks and their csoft interactions are described by the boosted heavy quark effective theory (bHQET) [36, 37], which can be directly obtained from $\operatorname{SCET}_{\mathrm{M}}[38,39]$.

The original collinear parts for the heavy quark sectors in eq. (6.4) can be refactorized as

$$
\begin{aligned}
& C_{n}\left(p_{+}, m^{2}, \mu, \nu_{+}\right)=C_{m}\left(m^{2}, \mu\right) B_{n}\left(p_{+}, m^{2}, \mu, \nu_{+}\right) \\
& C_{\bar{n}}\left(p_{-}^{\prime}, m^{2}, \mu, \nu_{-}\right)=C_{m}\left(m^{2}, \mu\right) B_{\bar{n}}\left(p_{-}^{\prime}, m^{2}, \mu, \nu_{-}\right) .
\end{aligned}
$$

Here $C_{m}$ is the result of integrating out the collinear interactions. At NLO in $\alpha_{s}$, it is given as $[37,40,41]$

$$
C_{m}\left(m^{2}, \mu\right)=1+\frac{\alpha_{s} C_{F}}{4 \pi}\left(\frac{1}{2} \ln \frac{\mu^{2}}{m^{2}}+\frac{1}{2} \ln ^{2} \frac{\mu^{2}}{m^{2}}+2+\frac{\pi^{2}}{12}\right) .
$$

In eqs. (6.12) and (6.13), $B_{n}$ and $B_{\bar{n}}$ are the $n$-csoft and $\bar{n}$-csoft functions in bHQET. As we will show, the csoft functions depend on the rapidity scales, which can be chosen to be of order $Q \zeta$ to minimize large rapidity logarithms. This choice of the scale corresponds to the size of the largest component of the csoft momentum.

For $F_{\mathrm{SCET}}$ at $\mu \ll m$, we also have to introduce ultrasoft (usoft) interactions, with the usoft momentum scaling as $p_{u s} \sim Q \zeta(\lambda, \lambda, \lambda)$. The usoft interactions are decoupled from both the csoft parts. And the resulting usoft function has the same form as the soft function $S_{n \bar{n}}$ in eq. (6.4). The only difference is that it consists of usoft gluons now. Let us consider the transition from the soft part to the usoft part systematically. In calculating the soft function $S_{n \bar{n}}$ at higher orders in $\alpha_{s}$, we have to subtract the usoft contributions to avoid double counting. Since the subtracted usoft contribution is always the same as the soft contribution, the overall contribution to the soft function should vanish to all orders in $\alpha_{s}$. Therefore, when $\mu \ll m$, we have the usoft part, instead of the soft part. As a result, the soft function $S_{n \bar{n}}$ in eq. (6.4) is replaced with the usoft function.

Finally $F_{\mathrm{SCET}}$ for $\mu \ll m$ can be factorized as

$$
F_{\mathrm{SCET}}\left(Q^{2}, m^{2}, \mu\right)=\left[C_{m}\left(m^{2}, \mu\right)\right]^{2} \cdot B_{n}\left(p_{+}, m^{2}, \mu, \nu_{+}\right) B_{\bar{n}}\left(p_{-}^{\prime}, m^{2}, \mu, \nu_{-}\right) Y_{n \bar{n}}\left(\mu, \nu_{+}, \nu_{-}\right) .
$$



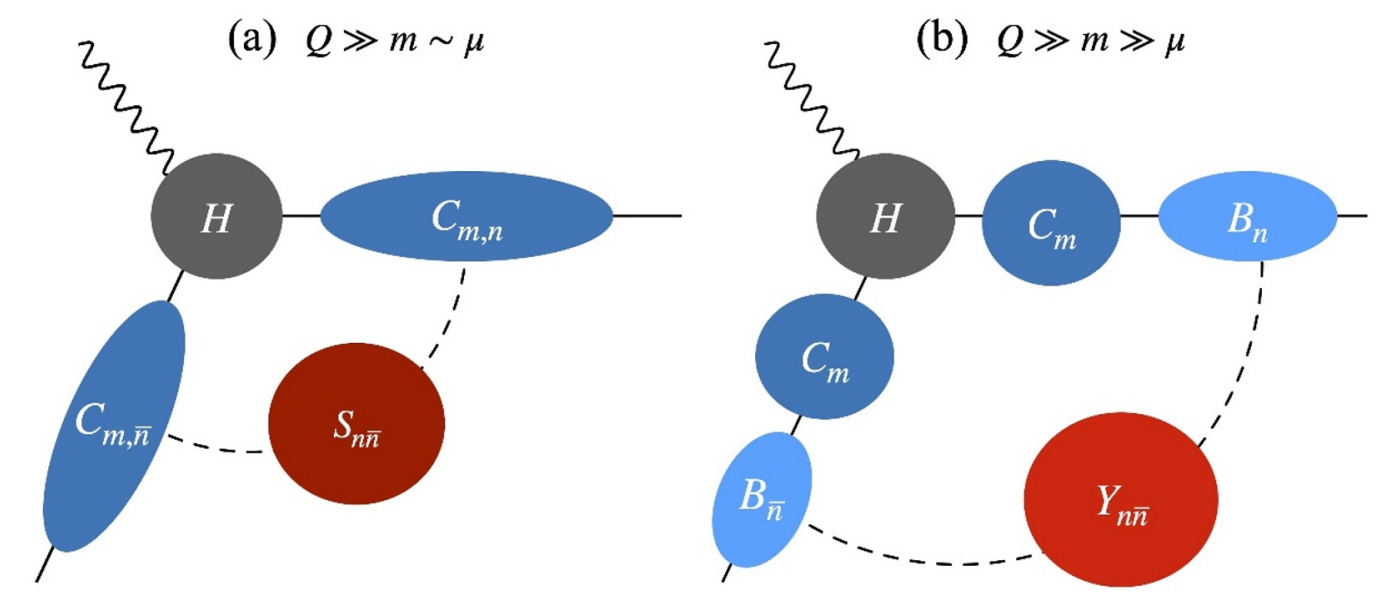

Figure 7. Schematic picture of the factorization structure in the heavy-to-heavy form factor: (a) For $\mu \sim m$, the form factor consists of the hard, collinear and soft functions. (b) For $\mu \ll m$, the collinear interactions with $p^{2} \sim m^{2}$ are integrated out to yield $C_{m}$. As a result, we have the csoft functions, $B_{n}$ and $B_{\bar{n}}$, and the usoft function $Y_{n \bar{n}}$.

Here $Y_{n \bar{n}}$ is the usoft function, which is the same as $S_{n \bar{n}}$ in eq. (3.25). This factorization with $\mu \ll m$ can be also useful for the elecroweak Sudakov form factor involving top quarks or the semi-inclusive deep inelastic scattering initiated by a heavy quark when an outgoing heavy quark (hadron) has small $p_{T}$ compared with the heavy quark mass. The structure of the factorization for the form factor is illustrated in figure 7 and is compared to the case for $\mu \sim m$.

The irreducible one-loop contribution to csoft function $B_{n}$ can be inferred from the collinear one-loop calculation for the heavy quark in eq. (6.1). Taking the gluon loop momentum to the csoft limit, $k^{\mu} \sim Q \zeta\left(1, \lambda, \lambda^{2}\right)$, the naive one-loop contribution to $B_{n}$ is written as ${ }^{5}$

$$
\tilde{M}_{c s}^{m}=-\frac{\alpha_{s} C_{F}}{2 \pi} \frac{\left(\mu^{2} e^{\gamma_{\mathrm{E}}}\right)^{\epsilon}}{\Gamma(1-\epsilon)} \int_{0}^{\infty} \frac{d x}{x} \int_{0}^{\infty} d \mathbf{k}_{\perp}^{2} \frac{\left(\mathbf{k}_{\perp}^{2}\right)^{-\epsilon}}{\mathbf{k}_{\perp}^{2}+x^{2} m^{2}} .
$$

Here $x=k_{+} / p_{+} \sim \zeta$ is a small quantity, hence the upper limit of $x$ is set to infinity. And $\mathbf{k}_{\perp}^{2}$ and $x^{2} m^{2}$ is power-counted as $\mathcal{O}\left(Q^{2} \zeta^{2} \lambda^{2}\right)$. Eq. (6.16) seems to possess the rapidity divergence as $x \rightarrow \infty$. However, it turns out to be the UV divergence, $1 / \epsilon_{\mathrm{UV}}$, due to the presence of the heavy quark mass (the term $x^{2} m^{2}$ in the integral of $\mathbf{k}_{\perp}^{2}$ ). Therefore eq. (6.16) does not contain the rapidity divergence as in the collinear case.

Instead, the rapidity divergence comes from the zero-bin contribution when the loop momentum $k$ becomes usoft. Since the usoft momentum scales as $k_{u s} \sim Q \zeta(\lambda, \lambda, \lambda)$, the momentum fraction $x$ in eq. (6.16) gets further suppressed as $\mathcal{O}(\zeta \lambda)$ and the term $x^{2} m^{2}$

\footnotetext{
${ }^{5}$ This calculation should be performed in bHQET. If we set $v_{n}^{\perp}=0, v_{n}=\left(\frac{p_{+}}{m} \frac{n^{\mu}}{2}+\frac{m}{p_{+}} \frac{\bar{n}^{\mu}}{2}\right)$, then the heavy quark propagator in bHQET becomes

$$
\frac{1}{v_{n} \cdot k} \propto \frac{1}{\mathbf{k}_{\perp}^{2}+x^{2} m^{2}}
$$

where the loop momentum satisfies the on-shell condition, $k_{-}=\mathbf{k}_{\perp}^{2} / k_{+}$after integraing over $k_{-}$in the complex plane.
} 
in the integral of $\mathbf{k}_{\perp}^{2}$ can be ignored. So the zero-bin contribution ends up with eq. (3.16), and it reads ${ }^{6}$

$$
M_{c s}^{\varnothing}=-\frac{\alpha_{s} C_{F}}{2 \pi} \frac{\left(\mu^{2} e^{\gamma_{\mathrm{E}}}\right)^{\epsilon}}{\Gamma(1-\epsilon)}\left(\frac{\nu_{+}}{p_{+}}\right)^{\eta_{+}} \int_{0}^{\infty} \frac{d x}{x^{1+\eta_{+}}} \int_{0}^{\infty} d \mathbf{k}_{\perp}^{2}\left(\mathbf{k}_{\perp}^{2}\right)^{-1-\epsilon}
$$

Therefore, the regular csoft (irreducible) one-loop contribution is given as

$$
\begin{aligned}
M_{c s}^{m} & =\tilde{M}_{c s}^{m}-M_{c s}^{\varnothing} \\
& =-\frac{\alpha_{s} C_{F}}{4 \pi}\left[\frac{1}{\epsilon_{\mathrm{UV}}^{2}}-\frac{1}{\epsilon_{\mathrm{IR}}^{2}}+\left(\frac{1}{\epsilon_{\mathrm{UV}}}-\frac{1}{\epsilon_{\mathrm{IR}}}\right) \ln \frac{\mu^{2}}{m^{2}}-\left(\frac{2}{\eta_{+}}+2 \ln \frac{\nu_{+}}{p_{+}}\right)\left(\frac{1}{\epsilon_{\mathrm{UV}}}-\frac{1}{\epsilon_{\mathrm{IR}}}\right)\right] .
\end{aligned}
$$

For the detailed calculation, we refer to appendix. B.2. The self-energy contribution to $B_{n}$ is given as $\left(Z_{h}^{(1)}+R_{h}^{(1)}\right) / 2$, where the heavy quark wave function renormalization and the residue at one loop in bHQET are given by

$$
Z_{h}^{(1)}=\frac{\alpha_{s} C_{F}}{2 \pi} \frac{1}{\epsilon_{\mathrm{UV}}}, \quad R_{h}^{(1)}=-\frac{\alpha_{s} C_{F}}{2 \pi} \frac{1}{\epsilon_{\mathrm{IR}}}
$$

Finally, the bare one-loop contribution to $B_{n}$ is given as

$$
\begin{aligned}
B_{n}^{(1)}\left(p_{+}, m^{2}, \mu, \nu_{+}\right) & =M_{c s}^{m}+\frac{1}{2}\left(Z_{h}^{(1)}+R_{h}^{(1)}\right) \\
& =\frac{\alpha_{s} C_{F}}{4 \pi}\left[-\frac{1}{\epsilon_{\mathrm{UV}}^{2}}+\frac{1}{\epsilon_{\mathrm{IR}}^{2}}+\left(\frac{1}{\epsilon_{\mathrm{UV}}}-\frac{1}{\epsilon_{\mathrm{IR}}}\right)\left(\frac{2}{\eta_{+}}+2 \ln \frac{\nu_{+}}{p_{+}}-\ln \frac{\mu^{2}}{m^{2}}+1\right)\right] .
\end{aligned}
$$

The csoft function $B_{\bar{n}}^{(1)}$ can be obtained by replacing $\left\{p_{+}, \eta_{+}, \nu_{+}\right\}$in $B_{n}^{(1)}$ with $\left\{p_{-}^{\prime}, \eta_{-}, \nu_{-}\right\}$.

If the IR divergence is regularized using the gluon mass $M$ that scales as $Q \zeta \lambda$, the renormalized NLO result for $B_{n}$ is expressed as

$$
B_{n}\left(p_{+}, m^{2}, \mu, \nu_{+}\right)=1+\frac{\alpha_{s} C_{F}}{2 \pi}\left[\ln \frac{\mu^{2}}{M^{2}}\left(\ln \frac{\nu_{+} m}{p_{+} M}-\ln \frac{\mu}{M}+\frac{1}{2}\right)+\frac{1}{4} \ln ^{2} \frac{\mu^{2}}{M^{2}}+\frac{\pi^{2}}{24}\right],
$$

from which, we can determine the characteristic rapidity scales for the csoft functions to minimize the large logarithms, and they are given by

$$
\nu_{c s}^{+} \sim \frac{p_{+} M}{m} \sim Q \zeta, \quad \nu_{\overline{c s}}^{-} \sim \frac{p_{-}^{\prime} M}{m} \sim Q \zeta .
$$

These scales correspond to the sizes of the largest components of the $n$ - and $\bar{n}$-csoft momenta. For the NLO result of the usoft function with the gluon mass, we refer to eq. (A.5).

The anomalous dimensions for the factorized functions with respect to $\mu$ and $\nu$ scales are defined in eq. (A.6). We list the anomalous dimensions for the factorized functions in

\footnotetext{
${ }^{6}$ When matched onto bHQET to describe the csoft interactions, the collinear Wilson line $W_{n}$ becomes $W_{n}^{c s}$, which has the same form as $W_{n}$ with the collinear gluon $A_{n}$ replaced by the csoft gluon $A_{n}^{c s}$. So the same rapidity regulator for the csoft calculation can be used in $W_{n}^{c s}$.
} 
eq. (6.15) as well as the hard function $H$ at order $\alpha_{s}$. The $\mu$-anomalous dimensions are given as

$$
\begin{array}{rlrl}
\gamma_{H} & =\frac{\alpha_{s} C_{F}}{2 \pi}\left(-2 \ln \frac{\mu^{2}}{Q^{2}}-3\right), & \gamma_{m}=\frac{1}{\left[C_{m}\right]^{2}} \frac{d\left[C_{m}\right]^{2}}{d \ln \mu}=\frac{\alpha_{s} C_{F}}{2 \pi}\left(2 \ln \frac{\mu^{2}}{m^{2}}+1\right), \\
\gamma_{c s}^{\mu}=\frac{1}{B_{n}} \frac{d B_{n}}{d \ln \mu}=\frac{\alpha_{s} C_{F}}{2 \pi}\left(2 \ln \frac{\nu_{+} m}{p_{+} \mu}+1\right), & \gamma_{\overline{c s}}^{\mu}=\frac{1}{B_{\bar{n}}} \frac{d B_{\bar{n}}}{d \ln \mu}=\frac{\alpha_{s} C_{F}}{2 \pi}\left(2 \ln \frac{\nu_{-} m}{p_{-}^{\prime} \mu}+1\right), \\
\gamma_{u s}^{\mu}=\frac{\alpha_{s} C_{F}}{\pi} \ln \frac{\mu^{2}}{\nu_{+} \nu_{-}} . &
\end{array}
$$

The nonzero $\nu$-anomalous dimensions appear in the csoft and usoft functions, and they are given by

$$
\gamma_{c s}^{\nu_{+}}=\gamma_{\overline{c s}}^{\nu_{-}}=\frac{\alpha_{s} C_{F}}{2 \pi} \ln \frac{\mu^{2}}{M^{2}}, \quad \gamma_{u s}^{\nu_{+}}=\gamma_{u s}^{\nu_{-}}=-\frac{\alpha_{s} C_{F}}{2 \pi} \ln \frac{\mu^{2}}{M^{2}} .
$$

With $Q^{2}=p_{+} p_{-}^{\prime}$, the sum of the anomalous dimensions cancel:

$$
\begin{aligned}
& \gamma_{H}^{\mu}+\gamma_{m}^{\mu}+\gamma_{c s}^{\mu}+\gamma_{\overline{c s}}^{\mu}+\gamma_{u s}^{\mu}=0, \\
& \gamma_{c s}^{\nu_{+}}+\gamma_{u s}^{\nu_{+}}=0, \quad \gamma_{\overline{c s}}^{\nu_{-}}+\gamma_{u s}^{\nu_{-}}=0 .
\end{aligned}
$$

This guarantees that the form factor is independent of $\mu$ and $\nu$. The resummation of the large logarithms using the anomalous dimensions to NLL accuracy is presented in appendix A.3.

Combining all the ingredients of the factorized functions in eqs. (6.4) and (6.15), the (bare) one-loop result of the form factor in SCET is written as

$$
\begin{aligned}
& F_{\mathrm{SCET}}^{(1)}\left(Q^{2}, m^{2}, \mu\right)= C_{m, n}^{(1)}+C_{m, \bar{n}}^{(1)}+S_{n \bar{n}}^{(1)} \\
&=2 C_{m}^{(1)}+B_{n}^{(1)}+B_{\bar{n}}^{(1)}+Y_{n \bar{n}}^{(1)} \\
&=\frac{\alpha_{s} C_{F}}{2 \pi}\left[\frac{1}{\epsilon_{\mathrm{UV}}^{2}}+\frac{1}{\epsilon_{\mathrm{UV}}}\left(\ln \frac{\mu^{2}}{p_{+} p_{-}^{\prime}}+\frac{3}{2}\right)-\frac{1}{\epsilon_{\mathrm{IR}}}\left(\ln \frac{m^{2}}{p_{+} p_{-}^{\prime}}+1\right)\right. \\
&\left.+\frac{1}{2} \ln ^{2} \frac{\mu^{2}}{m^{2}}+\frac{1}{2} \ln \frac{\mu^{2}}{m^{2}}+2+\frac{\pi^{2}}{12}\right],
\end{aligned}
$$

where $p_{+} p_{-}^{\prime}=Q^{2}$ and we used a massless gluon. The rapidity divergences exactly cancel between the collinear and soft parts as well as between the csoft and the usoft parts.

Combining the hard function in eq. (5.2) and the renormalized result of eq. (6.27), in the limit $Q \gg m$, we also show the one-loop result of the form factor in full QCD as ${ }^{7}$

$$
F^{(1)}\left(Q^{2}, m^{2}\right)=\frac{\alpha_{s} C_{F}}{2 \pi}\left[-2\left(1+\ln \frac{m^{2}}{Q^{2}}\right)\left(\frac{1}{\epsilon_{\mathrm{IR}}}+\ln \frac{\mu^{2}}{Q^{2}}\right)-\ln \frac{m^{2}}{Q^{2}}+\ln ^{2} \frac{m^{2}}{Q^{2}}-4+\frac{\pi^{2}}{3}\right] .
$$

This result coincides with ref. [27].

\footnotetext{
${ }^{7}$ Here the $\mu$-dependence is due to the dimensional regularization of the IR divergence, not a renormalization effect.
} 


\subsection{Heavy-to-light form factor}

We can also describe the form factor in the weak transition of a heavy quark to a light quark. When the transferred momentum is much larger than the heavy quark mass, i.e, $Q \gg m$, the heavy-to-light form factor for the weak current such as $\bar{q} \gamma^{\mu}\left(1-\gamma_{5}\right) Q$ can be matched onto SCET as

$$
F_{h l}\left(Q^{2}, m^{2}\right)=H\left(Q^{2}, \mu\right) F_{\mathrm{SCET}}^{h l}\left(Q^{2}, m^{2}, \mu\right) .
$$

Here the hard function is the same as in the light form factor as well as in the heavy-toheavy form factor.

The factorization of $F_{\mathrm{SCET}}^{h l}$ can be easily inferred from our analyses on the light-to-light and the heavy-to-heavy cases. For $\mu \sim m, F_{\mathrm{SCET}}^{h l}$ can be factorized as

$$
F_{\mathrm{SCET}}^{h l}\left(Q^{2}, m^{2}, \mu \sim m\right)=C_{n}\left(p_{+}, \mu, \nu_{+}\right) C_{m, \bar{n}}\left(p_{-}^{\prime}, m^{2}, \mu, \nu_{-}\right) S_{n \bar{n}}\left(\mu, \nu_{+}, \nu_{-}\right) .
$$

Here $C_{n}$ is the collinear function for the light quark in eqs. (3.15) and (5.3), and $C_{m, \bar{n}}$ is the $\left(\bar{n}\right.$-)collinear function for the heavy quark in eq. (6.4). $S_{n \bar{n}}$ is the universal soft function for the back-to-back current.

For $\mu \ll m$, we need to integrate out the collinear interaction with offshellness $\sim m^{2}$ in the heavy quark sector, which gives $C_{m}\left(m^{2}, \mu\right)$ in eq. (6.14). The SCET form factor can be written as

$$
F_{\mathrm{SCET}}^{h l}\left(Q^{2}, m^{2}, \mu \ll m\right)=C_{m}\left(m^{2}, \mu\right) \cdot C_{n}\left(p_{+}, \mu, \nu_{+}\right) B_{\bar{n}}\left(p_{-}^{\prime}, m^{2}, \mu, \nu_{-}\right) Y_{n \bar{n}}\left(\mu, \nu_{+}, \nu_{-}\right),
$$

where $B_{\bar{n}}$ and $Y_{n \bar{n}}$ are the csoft and the usoft functions introduced in section 6.1.2. And the collinear function for the light quark here describes the interactions with the offshellness $p^{2} \ll m^{2}$. The structure of the factorization is illustrated in figure 8 . The resummed result for the form factor is presented in appendix A.4.

In eq. (6.31), the behavior at the scale $\mu \ll m$ is described by $C_{n}, B_{\bar{n}}$ and $Y_{n \bar{n}}$. When we combine the three pieces as $f_{h l}=C_{n} \cdot B_{\bar{n}} \cdot Y_{n \bar{n}}$, the NLO result in $\alpha_{s}$ is given by

$$
\begin{aligned}
f_{h l}\left(Q^{2}, m^{2}, \mu\right) & =1+C_{n}^{(1)}+B_{\bar{n}}^{(1)}+Y_{n \bar{n}}^{(1)} \\
& =1+\frac{\alpha_{s} C_{F}}{4 \pi}\left[\frac{1}{\epsilon_{\mathrm{UV}}^{2}}-\frac{1}{\epsilon_{\mathrm{IR}}^{2}}+\left(\frac{1}{\epsilon_{\mathrm{UV}}}-\frac{1}{\epsilon_{\mathrm{IR}}}\right)\left(2 \ln \frac{\mu m}{p_{+} p_{-}^{\prime}}+\frac{5}{2}\right)\right],
\end{aligned}
$$

with $Q=p_{+} p_{-}^{\prime}$. There is no rapidity divergence in this combination, as expected. The anomalous dimension is given by

$$
\gamma_{f_{h l}}=\frac{\alpha_{s} C_{F}}{2 \pi}\left(2 \ln \frac{\mu m}{p_{+} p_{-}^{\prime}}+\frac{5}{2}\right)=\frac{\alpha_{s} C_{F}}{2 \pi}\left(2 \ln \frac{\mu m}{Q^{2}}+\frac{5}{2}\right) .
$$

Since the form factor in QCD is scale invariant, $\gamma_{f_{h l}}$ satisfies

$$
\gamma_{H}+\frac{1}{2} \gamma_{m}+\gamma_{f_{h l}}=0,
$$

where $\gamma_{H}$ and $\gamma_{m}$ at order $\alpha_{s}$ are given in eq. (6.23). 
(a) $Q \gg m \gg \mu$

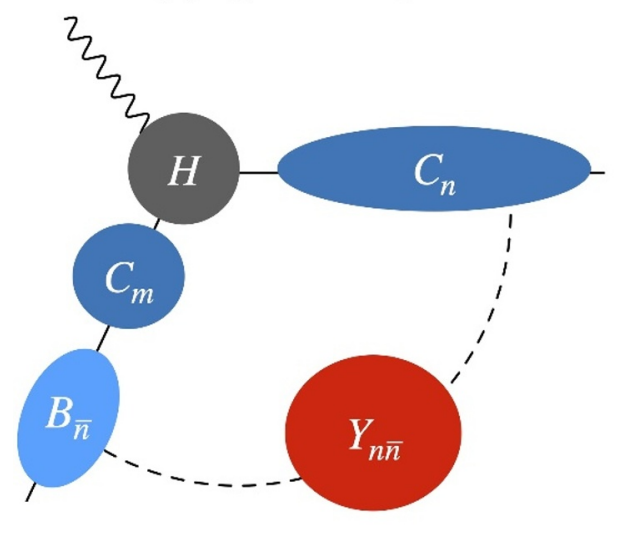

(b) $Q \sim m \gg \mu$

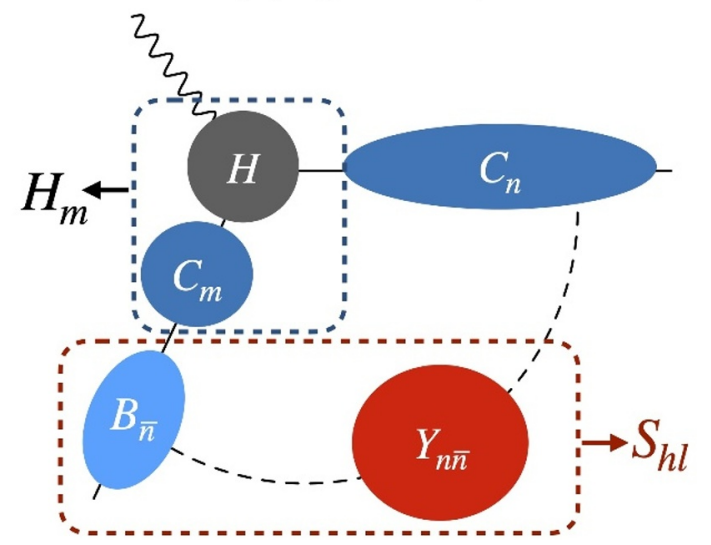

Figure 8. Schematic structure of the factorization for the heavy-to-light form factor: (a) For $Q \gg m \gg \mu$, the incoming heavy quark is boosted and described by $C_{m}$ and $B_{\bar{n}}$. Here $B_{\bar{n}}, Y_{n \bar{n}}$ and $C_{n}$ have almost the same offshellness $\left(p^{2} \sim \mu^{2} \ll m^{2}\right)$, but they are separated by the rapidities. (b) The form factor with a static heavy quark. Here $Q \sim m$ and the heavy quark experiences the soft interaction at lower energy scale $\mu$. So $H$ and $C_{m}$ can be merged into a new hard function $H_{m}$. And $B_{\bar{n}}$ and $Y_{n \bar{n}}$ can be combined into a new soft function $S_{h l}$.

Interestingly enough, the factorized result of $F_{h l}$ in eq. (6.29) with eq. (6.31) can be applied to the $B$ decay in the rest frame of the $B$ meson, where the factorization scale $\mu$ is much smaller than the heavy quark mass $m$. Suppose that the static heavy quark with the momentum $p^{\prime}$ decays to an energetic light quark with the momentum $p$ that has a maximal energy. In this case, the transferred momentum squared is given as

$$
q^{2}=\left(p-p^{\prime}\right)^{2}=m^{2}-2 p \cdot p^{\prime}=m^{2}-Q^{2}=0 .
$$

Here we set $2 p \cdot p^{\prime} \equiv Q^{2}$, which we have so far denoted the transferred momentum squared for the back-to-back current with very energetic quarks. The produced light quark can be described by the $n$-collinear field and its largest momentum component is given by $p_{+} \equiv \bar{n} \cdot p=m$.

Now we can find the factorized form factor for the static heavy quark decay from the energetic heavy-to-light form factor (eq. (6.29) employing eq. (6.31)). As depicted in figure 8 (b), since $Q^{2}=m^{2}, H$ in eq. (6.29) and $C_{m}$ in eq. (6.31) can be merged into a new hard function $H_{m}$, which is given by

$$
H_{m}\left(m^{2}, \mu\right)=H\left(Q^{2}=m^{2}, \mu\right) C_{m}\left(m^{2}, \mu\right)+\text { finite terms. }
$$

Here "the finite terms" denote the higher-order corrections in powers of $m^{2} / Q^{2}$ in the energetic heavy-to-light form factor. They are neglected when $Q^{2} \gg m^{2}$, which must be included here. However, the $\mu$-dependence resides genuinely in the combination of $H \cdot C_{m}$, hence the anomalous dimension is given as

$$
\gamma_{H_{m}}=\frac{1}{H_{m}} \frac{d}{d \ln \mu} H_{m}=-\frac{\alpha_{s} C_{F}}{2 \pi}\left(\ln \frac{\mu^{2}}{m^{2}}+\frac{5}{2}\right) .
$$

It is consistent with the result of the direct computation of the static heavy-to-light current $[1,2,42]$. 
Note that the overall description of the low energy behavior remains unchanged. Therefore, the combination of the factorized functions at the low scale, $f_{h l}$, is obtained from eq. (6.32) by replacing $Q^{2}\left(=p_{+} p_{-}^{\prime}\right)$ with $m^{2}$. And the sum of the anomalous dimension for $f_{h l}$ and $H_{m}$ cancels.

In the static heavy quark sector, the residual interactions after integrating out the offshellness $\sim m^{2}$ become soft and the mode scales as $p_{s} \sim m(\zeta, \zeta, \zeta)$ with $\zeta \sim \mu / m \ll 1$. Therefore, $B_{\bar{n}}$ and $Y_{n \bar{n}}$ in eq. (6.31) with the same rapidity scale $\nu_{-} \sim m \zeta$ are combined into the new soft function $S_{h l}$ as ${ }^{8}$

$$
S_{h l}\left(\mu, \nu_{+}\right)=B_{\bar{n}}\left(p_{-}^{\prime} \rightarrow m, m^{2}, \mu, \nu_{-}\right) Y_{n \bar{n}}\left(\mu, \nu_{+}, \nu_{-}\right) .
$$

This combination is illustrated in figure $8(\mathrm{~b})$. The bare one-loop result is given by

$$
S_{h l}^{(1)}\left(\mu, \nu_{+}\right)=\frac{\alpha_{s} C_{F}}{4 \pi}\left[\frac{1}{\epsilon_{\mathrm{UV}}^{2}}-\frac{1}{\epsilon_{\mathrm{IR}}^{2}}+\left(\frac{1}{\epsilon_{\mathrm{UV}}}-\frac{1}{\epsilon_{\mathrm{IR}}}\right)\left(-\frac{2}{\eta_{+}}-2 \ln \frac{\nu_{+}}{\mu}+1\right)\right] .
$$

Finally, in terms of the quantities in eqs. (6.36) and (6.38), we factorize the form factor for the static heavy quark decay as

$$
F_{h l}^{\text {stat }}\left(m^{2}, \mu\right)=H_{m}\left(m^{2}, \mu\right) C_{n}\left(p_{+}, \mu, \nu_{+}\right) S_{h l}\left(\mu, \nu_{+}\right) .
$$

When we sum over the soft and collinear contributions in eq. (6.40), the rapidity divergence cancels, and it ends up with the previous results $[1,2,42]$. However, the full resummation of the large logarithm of $\mu / m$ is completed by the rapidity evolution from $m$ to $\mu$, where $\mu \ll m$. We show the resummed result in appendix. A. 4 with the nonzero gluon mass $M \sim \mu$.

\section{Conclusions}

We have presented a new perspective regarding the origin of the rapidity divergence in SCET and its consistent treatment. The approach is based on applying the very idea of the effective theory to a system with a hierarchy of rapidities, that is, a system of collinear particles with large rapidity and soft particles with small rapidity, but with the same offshellness. The effective theory with soft modes is obtained by integrating out the collinear modes with large rapidity. The matching procedure exactly amounts to the zero-bin subtraction for the collinear sector in SCET. Since the rapidity divergence in the collinear sector comes from the subtraction of the overlapped soft contribution, the rapidity divergence in the soft sector has the opposite sign, which automatically guarantees the cancellation of the rapidity divergence in the total contribution. This is comparable to the usual matching procedure in the conventional effective theory with the degrees of freedom separated by the renormalization scale $\mu$.

In order to treat the rapidity divergences without any ambiguity, the main point in extracting the divergence in each sector is that we have to trace the same configurations

\footnotetext{
${ }^{8}$ Throughout this paper, we use the terminology "ultrasoft" to distinguish the mode from the soft mode with $p \sim(m, m, m)$ in describing the boosted heavy quark. In the static heavy quark decay, since the mode with the offshellness $\sim m^{2}$ is regarded as a hard mode, we call the mode with $p \sim m(\zeta, \zeta, \zeta)$ as the soft mode.
} 
both in the collinear and in the soft sectors. For example, the $n$-collinear Wilson line $W_{n}$ is obtained by the emission of the $n$-collinear gluons from the other part of the current, say, the $\bar{n}$-collinear sector from the back-to-back current. Therefore the corresponding soft sector in the matching, or the zero-bin subtraction, should come from the soft Wilson line $S_{\bar{n}}$ due to the emission of soft gluons from the $\bar{n}$-collinear sector, not $S_{n}$ which is built by the emission of the soft gluons from the $n$-collinear sector.

We employ the same rapidity regulator of the form $(\nu / \bar{n} \cdot k)^{\eta}$ both in the soft sector and in the collinear sector to regulate the rapidity divergence at large rapidity. When the current is not back-to-back, or when there is a soft quark involved, the same rapidity regulator is employed, but we use the appropriate expressions conforming to the regulator for the collinear part. In this process, the directional dependence enters in the soft rapidity regulator and it is essential in extracting the Lorentz invariants in the full theory when combined with the energy dependence from the collinear sector. Furthermore, unless the correct directional dependence is incorporated, an additional UV divergence is induced because the directional dependence can appear in the coefficients of the $1 / \epsilon_{\mathrm{UV}}$ pole [43]. With our prescription, the directional dependence is correctly implemented without any problem.

By extending the treatment of the rapidity divergence, we can associate independent rapidity scales for each collinear direction. Since physics should be independent of the rapidity divergence in any collinear direction, its cancellation in the combination of the soft and the collinear contributions gives a severe constraint on the structure of the effective theory. On the practical side, when the factorized collinear and soft parts share the same size of the rapidity scale, they can be combined to yield a new single function. It is illustrated in obtaining the soft function of the Sudakov form factor from the soft-collinear current, which is also confirmed by explicit calculations. This can be applied to various physical processes, in which the rapidity scales can be varied depending on physics so that part of the factorized parts can be combined.

We have extended the analysis on the rapidity divergence to the boosted heavy quark sector and found that the same mechanism of the divergence works. The resultant $\nu$ evolution enables us to fully resum large logarithms of $Q / m$ in the heavy quark form factors. Due to the presence of the heavy quark mass, the structure of the heavy quark form factors becomes more complicated and intriguing. We have consistently factorized the form factors matching onto $\mathrm{SCET}_{\mathrm{M}}$ and bHQET successively.

In SCET, it is important to include the evolution with respect to the rapidity scale because it yields the correct and the full resummation of the large logarithms when the rapidity divergence is involved. Therefore the deep understanding of the rapidity divergence is essential. And we hope that our analysis will be a cornerstone in developing deeper insight on the rapidity divergence. Our future plan is to apply this analysis systematically to various processes, with a variety of physical observables and beyond NLO calculations.

\section{A Resummation of large logarithms in Sudakov form factor}

We have presented the detailed computation of the Sudakov form factor for the back-toback and the soft-collinear currents at one loop. When pure dimensional regularization 
is employed with massless on-shell particles, the anomalous dimension with respect to the rapidity scale is IR divergent, which means that the Sudakov form factor is not factorizable, and unphysical. But we can consider the Sudakov form factor by putting a nonzero gauge boson mass. We resum large logarithms by employing the RG evolution with respect to the renormalization and the rapidity scales. It will be explicitly shown that the order of the $\mu$-evolution and the $\nu$-evolution is irrelevant.

We analyze the Sudakov form factor in terms of the back-to-back collinear current, and the soft-collinear current respectively. The second case can be obtained from the first by boosting to one of the collinear directions. Due to the Lorentz invariance, the Sudakov form factor and its evolution should be the same in both reference frames. However, the main theoretical interest here is that there are two different rapidity scales $\nu_{ \pm}$for the two collinear directions in the back-to-back current, ${ }^{9}$ while there is only one rapidity scale $\nu_{+}$ for one collinear direction in the soft-collinear current. Therefore the RG evolution in the two cases look seemingly different. We illustrate how the RG evolution is performed, and show that the resummed results for both cases are the same.

We also perform the resummation of large logarithms in the form factor for the heavyto-heavy current, where the incoming and the outgoing heavy quarks are boosted and they move in opposite direction. We show that the full resummation of the large logarithm of $Q / m$ is completed by the rapidity evolution. Finally, we present the resummed result of the form factor for the heavy-to-light current.

\section{A.1 Sudakov form factor for the light back-to-back current}

The amplitude of the back-to-back current at leading order is matched onto SCET as

$$
\left\langle p\left|J^{\mu}\right| p^{\prime}\right\rangle=\left\langle p\left|\bar{q}_{n} \gamma^{\mu} q_{\bar{n}}\right| p^{\prime}\right\rangle=H\left(Q^{2}, \mu\right) V_{\mathrm{SCET}}^{\mu}(\mu),
$$

where $V_{\text {SCET }}^{\mu}$ is the SCET amplitude for the current in eq. (2.1). The hard function $H\left(Q^{2}, \mu\right)$ at NLO in $\alpha_{s}$ is given by eq. (5.2), which is the same in the soft-collinear current. Since $V_{\mathrm{SCET}}^{\mu}$ is factorized, the Sudakov form factor in full QCD is written as

$$
F\left(Q^{2} ; M^{2}\right)=H\left(Q^{2}, \mu\right) C_{n}\left(p_{+}, \mu, \nu_{+} ; M^{2}\right) C_{\bar{n}}\left(p_{-}^{\prime}, \mu, \nu_{-} ; M^{2}\right) S_{n \bar{n}}\left(\mu, \nu_{+}, \nu_{-} ; M^{2}\right)
$$

where $Q^{2}=p_{+} p_{-}^{\prime}$ with the nonzero gauge boson mass $M$. From eqs. (3.11), (3.13) and (3.14), the renormalized NLO results for $C_{n}, C_{\bar{n}}$ and $S_{n \bar{n}}$ to one loop read

$$
\begin{gathered}
C_{n}\left(p_{+}, \mu, \nu_{+} ; M^{2}\right)=1+\frac{\alpha_{s} C_{F}}{4 \pi}\left[\ln \frac{\mu^{2}}{M^{2}}\left(2 \ln \frac{\nu_{+}}{p_{+}}+\frac{3}{2}\right)+\frac{9}{4}-\frac{\pi^{2}}{3}\right], \\
C_{\bar{n}}\left(p_{-}^{\prime}, \mu, \nu_{-} ; M^{2}\right)=1+\frac{\alpha_{s} C_{F}}{4 \pi}\left[\ln \frac{\mu^{2}}{M^{2}}\left(2 \ln \frac{\nu_{-}}{p_{-}^{\prime}}+\frac{3}{2}\right)+\frac{9}{4}-\frac{\pi^{2}}{3}\right], \\
S_{n \bar{n}}\left(\mu, \nu_{+}, \nu_{-} ; M^{2}\right)=1+\frac{\alpha_{s} C_{F}}{4 \pi}\left(\ln ^{2} \frac{\mu^{2}}{M^{2}}-2 \ln \frac{\mu^{2}}{M^{2}} \ln \frac{\nu_{+} \nu_{-}}{M^{2}}-\frac{\pi^{2}}{6}\right) .
\end{gathered}
$$

\footnotetext{
${ }^{9}$ Multiple rapidity scale evolution for the back-to-back case has been also discussed in refs. [18, 44].
} 
The $\mu$ and $\nu$ anomalous dimensions are defined as

$$
\gamma^{\mu}=\frac{1}{f} \frac{d}{d \ln \mu} f, \quad \gamma^{\nu}=\frac{1}{f} \frac{d}{d \ln \nu} f,
$$

for a given renormalized function $f$. Using eq. (A.6), we perform the RG evolutions to NLL accuracy, which resums large logarithms of order $\mathcal{O}(1)$ to all orders in $\alpha_{s}$. Here our power counting amounts to $\alpha_{s} L \sim \mathcal{O}(1)$, where $L$ denotes a large logarithm. The anomalous dimensions for the collinear and the soft parts are given as ${ }^{10}$

$$
\begin{aligned}
\gamma_{n}^{\mu} & =\frac{1}{2} \Gamma_{C}\left(\alpha_{s}\right) \ln \frac{\nu_{+}^{2}}{p_{+}^{2}}+\hat{\gamma}_{c}, & \gamma_{\bar{n}}^{\mu} & =\frac{1}{2} \Gamma_{C}\left(\alpha_{s}\right) \ln \frac{\nu_{-}^{2}}{p_{-}^{\prime 2}}+\hat{\gamma}_{c}, \quad \gamma_{s}^{\mu}=\Gamma_{C}\left(\alpha_{s}\right) \ln \frac{\mu^{2}}{\nu_{+} \nu_{-}}, \\
\gamma_{n}^{\nu_{+}} & =\gamma_{\bar{n}}^{\nu_{-}}=\frac{1}{2} \Gamma_{C}\left(\alpha_{s}\right) \ln \frac{\mu^{2}}{M^{2}}, & \gamma_{s}^{\nu_{+}} & =\gamma_{s}^{\nu_{-}}=-\frac{1}{2} \Gamma_{C}\left(\alpha_{s}\right) \ln \frac{\mu^{2}}{M^{2}},
\end{aligned}
$$

where $\hat{\gamma}_{c}=3 \alpha_{s} C_{F} /(4 \pi) . \Gamma_{C}\left(\alpha_{s}\right)$ is the cusp anomalous dimension [45, 46], which can be expanded in powers of $\alpha_{s}$ as $\Gamma_{C}=\sum_{k=0} \Gamma_{k}\left(\alpha_{s} / 4 \pi\right)^{k+1}$. To NLL accuracy, we need its first two coefficients,

$$
\Gamma_{0}=4 C_{F}, \quad \Gamma_{1}=4 C_{F}\left[\left(\frac{67}{9}-\frac{\pi^{2}}{3}\right) C_{A}-\frac{10}{9} n_{f}\right] .
$$

The anomalous dimension for the hard function $H\left(Q^{2}, \mu\right)$ is given by

$$
\gamma_{H}=-\Gamma_{c}\left(\alpha_{s}\right) \ln \frac{\mu^{2}}{Q^{2}}+\hat{\gamma}_{h}
$$

where $\hat{\gamma}_{h}=-6 \alpha_{s} C_{F} /(4 \pi)=-2 \hat{\gamma}_{c}$. As a consistency check, note that the following sums of the anomalous dimensions are zero.

$$
\begin{aligned}
& \gamma_{H}+\gamma_{n}^{\mu}+\gamma_{\bar{n}}^{\mu}+\gamma_{s}^{\mu}=0 \\
& \gamma_{n}^{\nu_{+}}+\gamma_{s}^{\nu_{+}}=0, \quad \gamma_{\bar{n}}^{\nu_{-}}+\gamma_{s}^{\nu_{-}}=0
\end{aligned}
$$

In eq. (A.2) the scales $\mu$ and $\nu_{ \pm}$in each factorized function are the factorization scales. In order to resum large logarithms, we need to consider the RG evolutions of the factorized functions from the factorization scales to their "characteristic scales". Here the characteristic scales mean the scales minimizing the large logarithms in the factorized function, and they are given by

$$
\begin{array}{ll}
\mu_{h} \sim Q, \quad \mu_{n} \sim \mu_{\bar{n}} \sim \mu_{s} \sim M, \\
\nu_{n}^{+} \sim p_{+}, \quad \nu_{\bar{n}}^{-} \sim p_{-}^{\prime}, \quad \nu_{s}^{ \pm} \sim M .
\end{array}
$$

Evolving $\mu$ from the characteristic scale to the factorization scale $\mu_{f}$, the factorized functions are expressed as

$$
\begin{aligned}
H\left(Q^{2}, \mu_{f}\right) & =U_{H}\left(\mu_{f}, \mu_{h}\right) H\left(Q^{2}, \mu_{h}\right), \\
C_{n}\left(p_{+}, \mu_{f}, \nu_{+} ; M^{2}\right) & =U_{n}\left(\mu_{f}, \mu_{n} ; \nu_{+}\right) C_{n}\left(p_{+}, \mu_{n}, \nu_{+} ; M^{2}\right), \\
C_{\bar{n}}\left(p_{-}^{\prime}, \mu_{f}, \nu_{-} ; M^{2}\right) & =U_{\bar{n}}\left(\mu_{f}, \mu_{\bar{n}} ; \nu_{-}\right) C_{\bar{n}}\left(p_{-}^{\prime}, \mu_{\bar{n}}, \nu_{-} ; M^{2}\right), \\
S_{n \bar{n}}\left(\mu_{f}, \nu_{ \pm} ; M^{2}\right) & =U_{s}\left(\mu_{f}, \mu_{s} ; \nu_{ \pm}\right) S_{n \bar{n}}\left(\mu_{s}, \nu_{ \pm} ; M^{2}\right) .
\end{aligned}
$$

\footnotetext{
${ }^{10}$ Here the expressions for the $\nu$-anomalous dimensions $\gamma^{\nu}$ in eq. (A.8) hold only for $\mu \sim M$.
} 
The evolution kernels to NLL accuracy are given as

$$
\begin{aligned}
U_{H}\left(\mu_{f}, \mu_{h}\right) & =\exp \left[-2 S_{\Gamma}\left(\mu_{f}, \mu_{h}\right)-a\left[\Gamma_{C}\right]\left(\mu_{f}, \mu_{f}\right) \ln \frac{\mu_{f}^{2}}{Q^{2}}+a\left[\hat{\gamma}_{h}\right]\left(\mu_{f}, \mu_{f}\right)\right], \\
U_{n}\left(\mu_{f}, \mu_{n} ; \nu_{+}\right) & =\exp \left[a\left[\Gamma_{C}\right]\left(\mu_{f}, \mu_{n}\right) \ln \frac{\nu_{+}}{p_{+}}+a\left[\hat{\gamma}_{c}\right]\left(\mu_{f}, \mu_{n}\right)\right], \\
U_{\bar{n}}\left(\mu_{f}, \mu_{\bar{n}} ; \nu_{-}\right) & =\exp \left[a\left[\Gamma_{C}\right]\left(\mu_{f}, \mu_{\bar{n}}\right) \ln \frac{\nu_{-}}{p_{-}^{\prime}}+a\left[\hat{\gamma}_{c}\right]\left(\mu_{f}, \mu_{\bar{n}}\right)\right], \\
U_{s}\left(\mu_{f}, \mu_{s} ; \nu_{ \pm}\right) & =\exp \left[2 S_{\Gamma}\left(\mu_{f}, \mu_{s}\right)+a\left[\Gamma_{C}\right]\left(\mu_{f}, \mu_{s}\right) \ln \frac{\mu_{f}^{2}}{\nu_{+} \nu_{-}}\right],
\end{aligned}
$$

where $S_{\Gamma}$ and $a[f]$ are defined as

$$
S_{\Gamma}\left(\mu_{1}, \mu_{2}\right)=\int_{\alpha_{s}\left(\mu_{2}\right)}^{\alpha_{s}\left(\mu_{1}\right)} \frac{d \alpha_{s}}{\beta\left(\alpha_{s}\right)} \Gamma_{C}\left(\alpha_{s}\right) \int_{\alpha_{s}\left(\mu_{1}\right)}^{\alpha_{s}} \frac{d \alpha_{s}^{\prime}}{\beta\left(\alpha_{s}^{\prime}\right)}, \quad a[f]\left(\mu_{1}, \mu_{2}\right)=\int_{\alpha_{s}\left(\mu_{2}\right)}^{\alpha_{s}\left(\mu_{1}\right)} \frac{d \alpha_{s}}{\beta\left(\alpha_{s}\right)} f\left(\alpha_{s}\right) .
$$

Here $\beta\left(\alpha_{s}\right)$ is the QCD beta function $\beta\left(\alpha_{s}\right)=d \alpha_{s} / d \ln \mu=-2 \alpha_{s} \sum_{k=0} \beta_{k}\left(\alpha_{s} / 4 \pi\right)^{k+1}$.

Evolving $\nu$ to the factorization scale $\nu_{f}$, the factorized functions are given as

$$
\begin{aligned}
C_{n}\left(p_{+}, \mu, \nu_{f}^{+} ; M^{2}\right) & =V_{n}\left(\nu_{f}^{+}, \nu_{n}^{+} ; \mu\right) C_{n}\left(p_{+}, \mu, \nu_{n}^{+} ; M^{2}\right), \\
C_{\bar{n}}\left(p_{-}^{\prime}, \mu, \nu_{f}^{-} ; M^{2}\right) & =V_{n}\left(\nu_{f}^{-}, \nu_{\bar{n}}^{-} ; \mu\right) C_{\bar{n}}\left(p_{-}^{\prime}, \mu, \nu_{\bar{n}}^{-} ; M^{2}\right), \\
S_{n \bar{n}}\left(\mu, \nu_{f}^{ \pm} ; M^{2}\right) & =V_{S}\left(\nu_{f}^{ \pm}, \nu_{s}^{ \pm} ; \mu\right) S_{n \bar{n}}\left(\mu, \nu_{s}^{ \pm} ; M^{2}\right),
\end{aligned}
$$

where the evolution kernels to NLL accuracy are given as

$$
\begin{aligned}
& V_{n}\left(\nu_{f}^{+}, \nu_{n}^{+} ; \mu\right)=\exp \left[\ln \frac{\nu_{f}^{+}}{\nu_{n}^{+}} \cdot a\left[\Gamma_{C}\right](\mu, M)\right], \\
& V_{\bar{n}}\left(\nu_{f}^{-}, \nu_{\bar{n}}^{-} ; \mu\right)=\exp \left[\ln \frac{\nu_{f}^{-}}{\nu_{\bar{n}}^{-}} \cdot a\left[\Gamma_{C}\right](\mu, M)\right], \\
& V_{S}\left(\nu_{f}^{ \pm}, \nu_{s}^{ \pm} ; \mu\right)=\exp \left[-\ln \frac{\nu_{f}^{ \pm}}{\nu_{s}^{ \pm}} \cdot a\left[\Gamma_{C}\right](\mu, M)\right] .
\end{aligned}
$$

In order to fully resum the large logarithms of $\mu / M$, the following relation is used [6]:

$$
\frac{d}{d \ln \mu} \gamma^{\nu}=\frac{d}{d \ln \nu} \gamma^{\mu} \propto \Gamma_{C}\left(\alpha_{s}\right)
$$

The $\nu$-anomalous dimensions in eq. (A.8) with a large logarithm is modified as

$$
\gamma_{n}^{\nu_{+}}=\gamma_{\bar{n}}^{\nu_{-}}=a\left[\Gamma_{C}\right](\mu, M), \quad \gamma_{s}^{\nu_{+}}=\gamma_{s}^{\nu_{-}}=-a\left[\Gamma_{C}\right](\mu, M) .
$$

In resumming the large logarithms, we may choose various paths for the evolutions with respect to $\mu$ and $\nu$ [6]. Let us first consider the $\nu$ evolution with fixed $\mu_{f}$, and then the $\mu$ evolution at $\nu_{i}=\left\{\nu_{n}^{+}, \nu_{n}^{-}, \nu_{s}^{ \pm}\right\}$. In this case the overall $\nu$-evolution kernel is given as

$$
\begin{aligned}
\ln V\left(\mu_{f}\right) & \equiv \ln \left[V_{n}\left(\nu_{f}^{+}, \nu_{n}^{+} ; \mu_{f}\right) \cdot V_{S}\left(\nu_{f}^{+}, \nu_{s}^{+} ; \mu_{f}\right)\right]+\ln \left[V_{\bar{n}}\left(\nu_{f}^{-}, \nu_{\bar{n}}^{-} ; \mu_{f}\right) \cdot V_{S}\left(\nu_{f}^{-}, \nu_{s}^{-} ; \mu_{f}\right)\right] \\
& =\ln \frac{\nu_{s}^{+} \nu_{s}^{-}}{\nu_{n}^{+} \nu_{\bar{n}}^{-}} \cdot a\left[\Gamma_{C}\right]\left(\mu_{f}, M\right) .
\end{aligned}
$$


Then we perform the $\mu$ evolution for the factorized functions at $\nu_{i}=\left\{\nu_{n}^{+}, \nu_{\bar{n}}^{-}, \nu_{s}^{ \pm}\right\}$. The overall $\mu$-evolution kernel is given as

$$
\begin{aligned}
\ln U\left(\nu_{i}\right) \equiv & \ln U_{H}\left(\mu_{f}, \mu_{h}\right)+\ln U_{n}\left(\mu_{f}, \mu_{n} ; \nu_{n}^{+}\right)+\ln U_{\bar{n}}\left(\mu_{f}, \mu_{\bar{n}} ; \nu_{\bar{n}}^{-}\right)+\ln U_{s}\left(\mu_{f}, \mu_{s} ; \nu_{s}^{ \pm}\right) \\
= & 2 S_{\Gamma}\left(\mu_{h}, \mu_{s}\right)+a\left[\hat{\gamma}_{c}\right]\left(\mu_{h}, \mu_{n}\right)+a\left[\hat{\gamma}_{c}\right]\left(\mu_{h}, \mu_{\bar{n}}\right)+\ln \frac{\mu_{h}^{2}}{Q^{2}} \cdot a\left[\Gamma_{C}\right]\left(\mu_{h}, \mu_{s}\right) \\
& +\ln \frac{\nu_{n}^{+} \nu_{\bar{n}}^{-}}{\nu_{s}^{+} \nu_{s}^{-}} \cdot a\left[\Gamma_{C}\right]\left(\mu_{f}, \mu_{s}\right)-\ln \frac{\nu_{n}^{+}}{p_{+}} \cdot a\left[\Gamma_{C}\right]\left(\mu_{n}, \mu_{s}\right)-\ln \frac{\nu_{\bar{n}}^{-}}{p_{-}^{\prime}} \cdot a\left[\Gamma_{C}\right]\left(\mu_{\bar{n}}, \mu_{s}\right) .
\end{aligned}
$$

Combining eqs. (A.32) and (A.33), we obtain the full exponentiation resumming the large logarithms to NLL accuracy. It is given as

$$
\begin{aligned}
\ln U\left(\nu_{i}\right) V\left(\mu_{f}\right)= & 2 S_{\Gamma}\left(\mu_{h}, \mu_{s}\right)+a\left[\hat{\gamma}_{c}\right]\left(\mu_{h}, \mu_{n}\right)+a\left[\hat{\gamma}_{c}\right]\left(\mu_{h}, \mu_{\bar{n}}\right)+\ln \frac{\mu_{h}^{2}}{Q^{2}} \cdot a\left[\Gamma_{C}\right]\left(\mu_{h}, \mu_{s}\right) \\
& -\ln \frac{\nu_{n}^{+} \nu_{\bar{n}}^{-}}{\nu_{s}^{+} \nu_{s}^{-}} \cdot a\left[\Gamma_{C}\right]\left(\mu_{s}, M\right)-\ln \frac{\nu_{n}^{+}}{p_{+}} \cdot a\left[\Gamma_{C}\right]\left(\mu_{n}, \mu_{s}\right)-\ln \frac{\nu_{\bar{n}}^{-}}{p_{-}^{\prime}} \cdot a\left[\Gamma_{C}\right]\left(\mu_{\bar{n}}, \mu_{s}\right) .
\end{aligned}
$$

Note that the dependence on the factorization scales is shown to be cancelled explicitly. In eq. (A.34), the first line is the result of the $\mu$ evolution only, and the second line reflects the rapidity scale evolution.

Since the renormalization scales $\mu_{n, \bar{n}, s}$ are given by $\sim M$, we can ignore the last two terms in the second line of eq. (A.34) at NLL accuracy because they are of order $\mathcal{O}\left(\alpha_{s}\right)$. Then the second line in eq. (A.34) can be approximated as

$$
-\ln \frac{\nu_{n}^{+} \nu_{\bar{n}}^{-}}{\nu_{s}^{+} \nu_{s}^{-}} \cdot a\left[\Gamma_{C}\right]\left(\mu_{s}, M\right) \approx-\ln \frac{\nu_{n}^{+} \nu_{\bar{n}}^{-}}{\nu_{s}^{+} \nu_{s}^{-}} \cdot \Gamma_{C}\left(\alpha_{s}\right) \ln \frac{\mu_{s}}{M} \approx-\frac{\alpha_{s}\left(\mu_{s}\right) C_{F}}{2 \pi} \ln \frac{Q^{2}}{M^{2}} \ln \frac{\mu_{s}^{2}}{M^{2}} .
$$

This is the most important missing ingredient in the exponentiation when we do not perform the rapidity evolution.

To check the path independence in the RG evolutions, let us consider a different path for the evolution. We perform the $\mu$ evolution at the scales $\nu_{f}^{ \pm}$first, and then the $\nu$ evolutions with the scales $\mu_{i}=\left\{\mu_{h}, \mu_{n}, \mu_{\bar{n}}, \mu_{s}\right\}$. In this case, the overall $\mu$-evolution kernel is given by

$$
\begin{aligned}
\ln U\left(\nu_{f}^{ \pm}\right)= & 2 S_{\Gamma}\left(\mu_{h}, \mu_{s}\right)+a\left[\hat{\gamma}_{c}\right]\left(\mu_{h}, \mu_{n}\right)+a\left[\hat{\gamma}_{c}\right]\left(\mu_{h}, \mu_{\bar{n}}\right)+\ln \frac{\mu_{h}^{2}}{Q^{2}} \cdot a\left[\Gamma_{C}\right]\left(\mu_{h}, \mu_{s}\right) \\
& -\ln \frac{\nu_{f}^{+}}{p_{+}} \cdot a\left[\Gamma_{C}\right]\left(\mu_{n}, \mu_{s}\right)-\ln \frac{\nu_{f}^{-}}{p_{-}^{\prime}} \cdot a\left[\Gamma_{C}\right]\left(\mu_{\bar{n}}, \mu_{s}\right) .
\end{aligned}
$$

And the overall $\nu$-evolution kernel at $\mu_{i}$ is obtained as

$$
\begin{aligned}
\ln V\left(\mu_{i}\right) & \equiv \ln \left[V_{n}\left(\nu_{f}^{+}, \nu_{n}^{+} ; \mu_{n}\right) \cdot V_{S}\left(\nu_{f}^{+}, \nu_{s}^{+} ; \mu_{s}\right)\right]+\ln \left[V_{\bar{n}}\left(\nu_{f}^{-}, \nu_{\bar{n}}^{-} ; \mu_{\bar{n}}\right) \cdot V_{S}\left(\nu_{f}^{-}, \nu_{s}^{-} ; \mu_{s}\right)\right] \\
& =\ln \frac{\nu_{f}^{+}}{\nu_{n}^{+}} \cdot a\left[\Gamma_{C}\right]\left(\mu_{n}, M\right)+\ln \frac{\nu_{f}^{-}}{\nu_{\bar{n}}^{-}} \cdot a\left[\Gamma_{C}\right]\left(\mu_{\bar{n}}, M\right)-\ln \frac{\nu_{f}^{+} \nu_{f}^{-}}{\nu_{s}^{+} \nu_{s}^{-}} \cdot a\left[\Gamma_{C}\right]\left(\mu_{s}, M\right) .
\end{aligned}
$$


Combining eqs. (A.36) and (A.37), the complete evolution kernel is given as

$$
\begin{aligned}
\ln V\left(\mu_{i}\right) U\left(\nu_{f}^{ \pm}\right)= & 2 S_{\Gamma}\left(\mu_{h}, \mu_{s}\right)+a\left[\hat{\gamma}_{c}\right]\left(\mu_{h}, \mu_{n}\right)+a\left[\hat{\gamma}_{c}\right]\left(\mu_{h}, \mu_{\bar{n}}\right)+\ln \frac{\mu_{h}^{2}}{Q^{2}} \cdot a\left[\Gamma_{C}\right]\left(\mu_{h}, \mu_{s}\right) \\
& -\ln \frac{\nu_{n}^{+} \nu_{\bar{n}}^{-}}{\nu_{s}^{+} \nu_{s}^{-}} \cdot a\left[\Gamma_{C}\right]\left(\mu_{s}, M\right)-\ln \frac{\nu_{n}^{+}}{p_{+}} \cdot a\left[\Gamma_{C}\right]\left(\mu_{n}, \mu_{s}\right)-\ln \frac{\nu_{\bar{n}}^{-}}{p_{-}^{\prime}} \cdot a\left[\Gamma_{C}\right]\left(\mu_{\bar{n}}, \mu_{s}\right) .
\end{aligned}
$$

It is the same as eq. (A.34), confirming the path independence of the RG evolutions with respect to $\mu$ and $\nu$.

\section{A.2 Sudakov form factor for the soft-collinear current}

The Sudakov form factor with the soft-collinear current is given in eq. (5.3), and is factorized as

$$
F\left(Q^{2}, M^{2}\right)=H\left(Q^{2}, \mu\right) C_{n}\left(p_{+}, \mu, \nu_{+} ; M^{2}\right) S_{q}\left(p_{-}^{\prime}, \mu, \nu_{+} ; M^{2}\right),
$$

where $Q^{2}=p_{+} p_{-}^{\prime}, p_{+} \sim Q^{2} / M$ and $p_{-}^{\prime} \sim M$. The soft function $S_{q}$ at NLO in $\alpha_{s}$ is given by

$$
S_{q}\left(p_{-}^{\prime}, \mu, \nu_{+} ; M^{2}\right)=1+\frac{\alpha_{s} C_{F}}{4 \pi}\left[\ln ^{2} \frac{\mu^{2}}{M^{2}}-2 \ln \frac{\mu^{2}}{M^{2}} \ln \frac{\nu_{+} p_{-}^{\prime}}{M^{2}}+\frac{9}{4}-\frac{\pi^{2}}{2}\right] .
$$

This soft function also can be obtained by combining $C_{\bar{n}}$ and $S_{n \bar{n}}$ from the back-to-back current as [See eq. (5.16).]

$$
S_{q}\left(p_{-}^{\prime}, \mu, \nu_{+} ; M^{2}\right)=C_{\bar{n}}\left(p_{-}^{\prime}, \mu, \nu_{-} ; M^{2}\right) S_{n \bar{n}}\left(\mu, \nu_{+}, \nu_{-} ; M^{2}\right) .
$$

Therefore the resummed result for eq. (A.39) can be obtained from the back-to-back case, starting from eq. (A.34). By identifying $\mu_{\bar{n}}=\mu_{s}$ and $\nu_{\bar{n}}^{-}=\nu_{s}^{-}$, we obtain the resummed result for the soft-collinear current. The exponentiation factor at NLL accuracy leads to

$$
\begin{aligned}
\ln F\left(Q^{2}, M^{2}\right)= & 2 S_{\Gamma}\left(\mu_{h}, \mu_{s}\right)+a\left[\hat{\gamma}_{c}\right]\left(\mu_{h}, \mu_{n}\right)+a\left[\hat{\gamma}_{c}\right]\left(\mu_{h}, \mu_{s}\right)+\ln \frac{\mu_{h}^{2}}{Q^{2}} \cdot a\left[\Gamma_{C}\right]\left(\mu_{h}, \mu_{s}\right) \\
& -\ln \frac{\nu_{n}^{+}}{\nu_{s}^{+}} \cdot a\left[\Gamma_{C}\right]\left(\mu_{s}, M\right)-\ln \frac{\nu_{n}^{+}}{p_{+}} \cdot a\left[\Gamma_{C}\right]\left(\mu_{n}, \mu_{s}\right),
\end{aligned}
$$

where $\mu_{n} \sim \mu_{s} \sim M$. Here the last term is power counted as $\mathcal{O}\left(\alpha_{s}\right)$ and ignored, and the second line in eq. (A.42) can be approximated as

$$
-\ln \frac{\nu_{n}^{+}}{\nu_{s}^{+}} \cdot a\left[\Gamma_{C}\right]\left(\mu_{s}, M\right) \approx-\ln \frac{Q^{2} / M}{M} \cdot \Gamma_{C}\left(\alpha_{s}\right) \ln \frac{\mu_{s}}{M} \approx-\frac{\alpha_{s} C_{F}}{2 \pi} \ln \frac{Q^{2}}{M^{2}} \ln \frac{\mu_{s}^{2}}{M^{2}} .
$$

This result is the same as the case for the back-to-back current in eq. (A.35). Therefore we confirm that the resummed result for the soft-collinear current is identical to that for the back-to-back current. It should hold because the Sudakov form factor is Lorentz invariant, but here we have proved explicitly the relation between a single-scale evolution and a two-scale evolution. 


\section{A.3 Sudakov form factor for the heavy-to-heavy current}

The factorized Sudakov form factor for the heavy-to-heavy current at the scale $\mu \ll m$ from eqs. (6.3) and (6.15) is given as

$$
\begin{aligned}
F\left(Q^{2}, m^{2} ; M^{2}\right)= & H\left(Q^{2}, \mu\right)\left[C_{m}\left(m^{2}, \mu\right)\right]^{2} \\
& \times B_{n}\left(p_{+}, m^{2}, \mu, \nu_{+} ; M^{2}\right) B_{\bar{n}}\left(p_{-}^{\prime}, m^{2}, \mu, \nu_{-} ; M^{2}\right) Y_{n \bar{n}}\left(\mu, \nu_{+}, \nu_{-} ; M^{2}\right) .
\end{aligned}
$$

Here the nonzero gluon mass scaling as $M \ll m$ is employed to regularize the IR divergence. The evolution of the hard function $H$ from the hard scale $\mu_{h} \sim Q$ to the factorization scale $\mu$ is given by eqs. (A.15) and (A.19). And the evolutions of the usoft function $Y_{n \bar{n}}$ are the same as the soft function $S_{n \bar{n}}$ for the form factor from the light quarks in eq. (A.2). So the $\mu$ - and $\nu$-evolution kernels are given by eq. (A.22) and (A.26) respectively.

To NLL accuracy, the $\mu$-anomalous dimensions for $\left[C_{m}\right]^{2}$ and $B_{n, \bar{n}}$ are listed as

$$
\begin{aligned}
& \gamma_{m}=\Gamma_{C}\left(\alpha_{s}\right) \ln \frac{\mu^{2}}{m^{2}}+\hat{\gamma}_{m}, \\
& \gamma_{c s}^{\mu}=\Gamma_{C}\left(\alpha_{s}\right)\left(-\frac{1}{2} \ln \frac{\mu^{2}}{M^{2}}+\ln \frac{\nu_{+} m}{p_{+} M}\right)+\hat{\gamma}_{c s}, \\
& \gamma_{\overline{c s}}^{\mu}=\Gamma_{C}\left(\alpha_{s}\right)\left(-\frac{1}{2} \ln \frac{\mu^{2}}{M^{2}}+\ln \frac{\nu_{-} m}{p_{-}^{\prime} M}\right)+\hat{\gamma}_{c s},
\end{aligned}
$$

where $\hat{\gamma}_{m}=\hat{\gamma}_{c s}=\alpha_{s} C_{F} /(2 \pi)$. The $\nu$-anomalous dimensions for $B_{n, \bar{n}}$ are given as

$$
\gamma_{c s}^{\nu_{+}}=\gamma_{\overline{c s}}^{\nu_{-}}=a\left[\Gamma_{C}\right](\mu, M)
$$

Using the anomalous dimensions, we evolve the factorized functions from the factorization scales $\left(\mu_{f}, \nu_{f}^{ \pm}\right)$to their characteristic scales to minimize large logarithms. The $\mu$-evolution kernels are given as

$$
\begin{gathered}
\ln U_{m}\left(\mu_{f}, \mu_{m}\right)=2 S_{\Gamma}\left(\mu_{f}, \mu_{m}\right)+\ln \frac{\mu_{f}^{2}}{m^{2}} a\left[\Gamma_{C}\right]\left(\mu_{f}, \mu_{m}\right)+a\left[\hat{\gamma}_{m}\right]\left(\mu_{f}, \mu_{m}\right), \\
\ln U_{c s}\left(\mu_{f}, \mu_{c s} ; \nu_{+}\right)=-S_{\Gamma}\left(\mu_{f}, \mu_{c s}\right)+\ln \frac{\nu_{+} m}{p_{+} \mu_{f}} a\left[\Gamma_{C}\right]\left(\mu_{f}, \mu_{c s}\right)+a\left[\hat{\gamma}_{c s}\right]\left(\mu_{f}, \mu_{c s}\right), \\
\ln U_{\overline{c s}}\left(\mu_{f}, \mu_{\overline{c s}} ; \nu_{-}\right)=-S_{\Gamma}\left(\mu_{f}, \mu_{\overline{c s}}\right)+\ln \frac{\nu_{-} m}{p_{-}^{\prime} \mu_{f}} a\left[\Gamma_{C}\right]\left(\mu_{f}, \mu_{\overline{c s}}\right)+a\left[\hat{\gamma}_{c s}\right]\left(\mu_{f}, \mu_{\overline{c s}}\right),
\end{gathered}
$$

where $\mu_{m} \sim m$ and $\mu_{c s} \sim \mu_{\overline{c s}} \sim M$. And the $\nu$-evolution kernels are given by

$$
\ln V_{c s}\left(\nu_{f}^{+}, \nu_{c s}^{+} ; \mu\right)=\ln \frac{\nu_{f}^{+}}{\nu_{c s}^{+}} a\left[\Gamma_{C}\right](\mu, M), \quad \ln V_{\overline{c s}}\left(\nu_{f}^{-}, \nu_{\overline{c s}}^{-} ; \mu\right)=\ln \frac{\nu_{f}^{-}}{\nu_{\overline{c s}}^{-}} a\left[\Gamma_{C}\right](\mu, M),
$$

where $\nu_{c s}^{+} \sim p_{+} M / m$ and $\nu_{\overline{c s}}^{-} \sim p_{-}^{\prime} M / m$.

Applying eqs. (A.49)-(A.52) and using the evolved results of $H$ and $Y_{n \bar{n}}$, we obtain the resummed result of the heavy-to-heavy form factor. The evolution of the form factor 
to NLL accuracy is given as

$$
\begin{aligned}
\ln F & \left(Q^{2}, m^{2} ; M^{2}\right)=2 S_{\Gamma}\left(\mu_{h}, \mu_{m}\right)+S_{\Gamma}\left(\mu_{c s}, \mu_{u s}\right)+S_{\Gamma}\left(\mu_{\overline{c s}}, \mu_{u s}\right)+\ln \frac{\mu_{h}^{2}}{Q^{2}} a\left[\Gamma_{C}\right]\left(\mu_{h}, \mu_{m}\right) \\
& +\ln \frac{m^{2}}{Q^{2}} a\left[\Gamma_{C}\right]\left(\mu_{m}, \mu_{u s}\right)+\ln \frac{p_{+} \mu_{c s}}{\nu_{c s}^{+} m} a\left[\Gamma_{C}\right]\left(\mu_{c s}, \mu_{u s}\right)+\ln \frac{p_{-}^{\prime} \mu_{\overline{c s}}}{\nu_{\overline{c s}}^{-} m} a\left[\Gamma_{C}\right]\left(\mu_{\overline{c s}}, \mu_{u s}\right) \\
& +\ln \frac{\nu_{u s}^{+} \nu_{u s}^{-}}{\nu_{c s}^{+} \nu_{\overline{c s}}^{-}} a\left[\Gamma_{C}\right]\left(\mu_{u s}, M\right)+\frac{C_{F}}{\beta_{0}}\left(\ln \frac{\alpha_{s}\left(\mu_{m}\right)}{\alpha_{s}\left(\mu_{h}\right)}+\frac{\alpha_{s}\left(\mu_{c s}\right)}{\alpha_{s}\left(\mu_{h}\right)}+\frac{\alpha_{s}\left(\mu_{\overline{c s}}\right)}{\alpha_{s}\left(\mu_{h}\right)}\right) .
\end{aligned}
$$

By suppressing the terms that are power-counted as $\mathcal{O}\left(\alpha_{s}\right)$ due to the fact that $\mu_{c s} \sim \mu_{\overline{c s}} \sim$ $\mu_{\text {us }} \sim M$, eq. (A.53) reads

$$
\begin{aligned}
\ln F\left(Q^{2}, m^{2} ; M^{2}\right)= & 2 S_{\Gamma}\left(\mu_{h}, \mu_{m}\right)+\ln \frac{m^{2}}{Q^{2}} a\left[\Gamma_{C}\right]\left(\mu_{m}, \mu_{u s}\right)+\ln \frac{\mu_{h}^{2}}{Q^{2}} a\left[\Gamma_{C}\right]\left(\mu_{h}, \mu_{m}\right) \\
& +\frac{C_{F}}{\beta_{0}}\left(\ln \frac{\alpha_{s}\left(\mu_{m}\right)}{\alpha_{s}\left(\mu_{h}\right)}+\ln \frac{\alpha_{s}\left(\mu_{c s}\right)}{\alpha_{s}\left(\mu_{h}\right)}+\ln \frac{\alpha_{s}\left(\mu_{\overline{c s}}\right)}{\alpha_{s}\left(\mu_{h}\right)}\right)+\ln \frac{\nu_{u s}^{+} \nu_{u s}^{-}}{\nu_{c s}^{+} \nu_{\overline{c s}}^{-}} a\left[\Gamma_{C}\right]\left(\mu_{u s}, M\right),
\end{aligned}
$$

where the first two terms in the right side are $\mathcal{O}\left(1 / \alpha_{s}\right)$ and the remaining terms are powercounted as $\mathcal{O}(1)$. The last term in eq. (A.54) is the result of the rapidity evolution. It can be written as

$$
\ln \frac{\nu_{u s}^{+} \nu_{u s}^{-}}{\nu_{c s}^{+} \nu_{\overline{c s}}^{-}} a\left[\Gamma_{C}\right]\left(\mu_{u s}, M\right) \sim \ln \frac{m^{2}}{p_{+} p_{-}^{\prime}} a\left[\Gamma_{C}\right]\left(\mu_{u s}, M\right) \approx \frac{\alpha_{s} C_{F}}{2 \pi} \ln \frac{m^{2}}{Q^{2}} \ln \frac{\mu_{u s}^{2}}{M^{2}} .
$$

It shows that the full resummation of $\ln Q / m$ in the form factor can be completed only after the rapidity evolution.

\section{A.4 Sudakov form factor for the heavy-to-light current}

With the nonzero gluon mass $M \sim \mu$, the resummation of the heavy-to-light form factor in eqs. (6.30) and (6.31) can be systematically performed by evolving with respec to $\mu$ and $\nu_{ \pm}$. It is straightforward to follow the same procedure as in the light-to-light and the heavy-to-heavy form factors,

When the form factor in SCET is obtained at the scale $\mu \sim m$, the $\mu$ - and $\nu$ renormalization behavior for each factorized function in eqs. (6.30) is the same as that of the light-to-light current. Therefore the resummed result is given by eqs. (A.34) and (A.38). For $\mu \ll m$, using the factorized result in eq. (6.31) with the gluon mass $M \ll m$, we obtain the resummed result at NLL accuracy as

$$
\begin{aligned}
\ln F_{h l}\left(Q^{2}, m^{2} ; M^{2}\right) & =2 S_{\Gamma}\left(\mu_{h}, \mu_{u s}\right)-S_{\Gamma}\left(\mu_{m}, \mu_{\overline{c s}}\right)+\ln \frac{\mu_{h}^{2}}{Q^{2}} a\left[\Gamma_{C}\right]\left(\mu_{h}, \mu_{u s}\right) \\
& -\frac{1}{2} \ln \frac{\mu_{m}^{2}}{m^{2}} a\left[\Gamma_{C}\right]\left(\mu_{m}, \mu_{\overline{c s}}\right)+\frac{C_{F}}{\beta_{0}}\left(\frac{1}{2} \ln \frac{\alpha_{s}\left(\mu_{m}\right)}{\alpha_{s}\left(\mu_{h}\right)}+\ln \frac{\alpha_{s}\left(\mu_{\overline{c s}}\right)}{\alpha_{s}\left(\mu_{h}\right)}+\frac{3}{2} \ln \frac{\alpha_{s}\left(\mu_{n}\right)}{\alpha_{s}\left(\mu_{h}\right)}\right) \\
& +\ln \frac{\nu_{u s}^{+} \nu_{u s}^{-}}{\nu_{n}^{+} p_{-}^{\prime}} a\left[\Gamma_{C}\right]\left(\mu_{u s}, M\right)-\ln \frac{\nu_{\overline{c s}}^{-}}{p_{-}^{\prime}} a\left[\Gamma_{C}\right]\left(\mu_{\overline{c s}}, M\right),
\end{aligned}
$$


where the characteristic scales for the factorized functions are given by

$$
\begin{aligned}
& \mu_{n} \sim \mu_{u s} \sim \mu_{\overline{c s}} \sim M, \\
& \nu_{n}^{+} \sim p_{+} \sim Q, \quad \nu_{\overline{c s}}^{-} \sim \frac{p_{-}^{\prime} M}{m} \sim \frac{Q M}{m}, \quad \nu_{u s}^{ \pm} \sim M .
\end{aligned}
$$

The last two terms in eq. (A.56) result from the rapidity evolution. Because $\mu_{u s} \sim \mu_{\overline{c s}} \sim M$, they can be simplified further as

$$
\begin{aligned}
& \ln \frac{\nu_{u s}^{+} \nu_{u s}^{-}}{\nu_{n}^{+} p_{-}^{\prime}} a\left[\Gamma_{C}\right]\left(\mu_{u s}, M\right)-\ln \frac{\nu_{\overline{c s}}^{-}}{p_{-}^{\prime}} a\left[\Gamma_{C}\right]\left(\mu_{\overline{c s}}, M\right) \\
& \quad \sim\left(\ln \frac{\nu_{u s}^{+}}{\nu_{n}^{+}}+\ln \frac{\nu_{u s}^{-}}{\nu_{\overline{c s}}^{-}}\right) a\left[\Gamma_{C}\right]\left(\mu_{u s}, M\right) \approx \frac{\alpha_{s}\left(\mu_{u s}\right) C_{F}}{2 \pi}\left(\ln \frac{M}{Q}+\ln \frac{m}{Q}\right) \ln \frac{\mu_{u s}^{2}}{M^{2}} .
\end{aligned}
$$

From the resummed result in eq. (A.56), we can consider the resummation of the form factor for the static heavy quark decay. Suppose that the light quark has a maximal energy, i.e., $p_{+}=m$. Then $Q^{2}=2 p \cdot p^{\prime}$ is given by $m^{2}$, and $\mu_{h, m}$ in eq. (A.56) becomes $\mu_{h}=\mu_{m} \sim m$. The characteristic usoft and csoft scales in eq. (A.56) are determined as $\mu_{u s}=\mu_{\overline{c s}} \sim M$ and $\nu_{u s}^{-}=\nu_{\overline{c s}}^{-} \sim M$. And the scales are unified as the soft scales for the static heavy quark decay. Therefore the resummed result for the static heavy quark is given as

$$
\begin{aligned}
\ln F_{h l}\left(m^{2} ; M^{2}\right)= & S_{\Gamma}\left(\mu_{h}, \mu_{s}\right)+\frac{1}{2} \ln \frac{\mu_{h}^{2}}{m^{2}} a\left[\Gamma_{C}\right]\left(\mu_{h}, \mu_{s}\right) \\
& +\frac{C_{F}}{\beta_{0}}\left(\ln \frac{\alpha_{s}\left(\mu_{s}\right)}{\alpha_{s}\left(\mu_{h}\right)}+\frac{3}{2} \ln \frac{\alpha_{s}\left(\mu_{n}\right)}{\alpha_{s}\left(\mu_{h}\right)}\right)+\ln \frac{\nu_{s}^{+}}{\nu_{n}^{+}} a\left[\Gamma_{C}\right]\left(\mu_{s}, M\right) .
\end{aligned}
$$

Here $\mu_{s} \sim M$, and the last term is the missing ingredient unless we consider the rapidity evolution. Since $\nu_{n}^{+} \sim m$ and $\nu_{s}^{+} \sim M$, it can be expressed as

$$
\ln \frac{\nu_{s}^{+}}{\nu_{n}^{+}} a\left[\Gamma_{C}\right]\left(\mu_{s}, M\right) \approx \frac{\alpha_{s}\left(\mu_{s}\right) C_{F}}{2 \pi} \ln \frac{M}{m} \ln \frac{\mu_{s}^{2}}{M^{2}} .
$$

\section{B One-loop calculations in the boosted heavy quark sector}

\section{B.1 Collinear one-loop calculation with the soft zero-bin mode $p_{s}^{\mu} \sim(m, m, m)$}

When the soft mode with $p_{s}^{\mu} \sim(m, m, m)$ is decoupled from the boosted heavy quark sector, we have to subtract the soft contribution from the naive collinear calculation for the heavy quark. The regular collinear one-loop calculation is given by $M_{n}^{m}=\tilde{M}_{n}^{m}-M_{n}^{\varnothing}$, as shown in eq. (6.2). It reads

$$
\begin{aligned}
M_{n}^{m}= & -\frac{\alpha_{s} C_{F}}{2 \pi} \frac{\left(\mu^{2} e^{\gamma_{\mathrm{E}}}\right)^{\epsilon}}{\Gamma(1-\epsilon)}\left[\int_{0}^{1} \frac{d x}{x}(1-x) \int_{0}^{\infty} \frac{d \mathbf{k}_{\perp}^{2}\left(\mathbf{k}_{\perp}^{2}\right)^{-\epsilon}}{\mathbf{k}_{\perp}^{2}+x^{2} m^{2}}-\int_{0}^{1} \frac{d x}{x} \int_{0}^{\infty} d \mathbf{k}_{\perp}^{2}\left(\mathbf{k}_{\perp}^{2}\right)^{-1-\epsilon}\right] \\
& +\frac{\alpha_{s} C_{F}}{2 \pi} \frac{\left(\mu^{2} e^{\gamma_{\mathrm{E}}}\right)^{\epsilon}}{\Gamma(1-\epsilon)}\left(\frac{\nu_{+}}{p_{+}}\right)^{\eta_{+}} \int_{1}^{\infty} d x x^{-1-\eta_{+}} \int_{0}^{\infty} d \mathbf{k}_{\perp}^{2}\left(\mathbf{k}_{\perp}^{2}\right)^{-1-\epsilon} .
\end{aligned}
$$


In the right side of eq. (B.1), the first term in the square bracket is the naive contribution $\left(\tilde{M}_{n}^{m}\right)$, and the remaining terms are the contributions from the zero-bin subtraction $\left(-M_{n}^{\varnothing}\right)$. Since the rapidity divergence arises as $x \rightarrow \infty$, we put the regulator only in the term in the second line of eq. (B.1).

We denote the first line and the second line of the right side in eq. (B.1) as $M_{n}^{m, A}$ and $M_{n}^{m, B}$ respectively. Then $M_{n}^{m, A}$ can be reorganized, and can be written as

$$
\begin{aligned}
M_{n}^{m, A}=-\frac{\alpha_{s} C_{F}}{2 \pi} & \frac{\left(\mu^{2} e^{\gamma_{\mathrm{E}}}\right)^{\epsilon}}{\Gamma(1-\epsilon)}\left[-\int_{0}^{1} d x \int_{0}^{\infty} \frac{d \mathbf{k}_{\perp}^{2}\left(\mathbf{k}_{\perp}^{2}\right)^{-\epsilon}}{\mathbf{k}_{\perp}^{2}+x^{2} m^{2}}\right. \\
& \left.+\int_{0}^{1} \frac{d x}{x} \int_{0}^{\infty} d \mathbf{k}_{\perp}^{2}\left(\mathbf{k}_{\perp}^{2}\right)^{-\epsilon}\left(\frac{1}{\mathbf{k}_{\perp}^{2}+x^{2} m^{2}}-\frac{1}{\mathbf{k}_{\perp}^{2}}\right)\right] .
\end{aligned}
$$

Here the first term in the square bracket gives a UV pole as $\mathbf{k}_{\perp}^{2}$ goes to infinity. In the second term, only the IR divergence survives. Then $M_{n}^{m, A}$ is given by

$$
M_{n}^{m, A}=\frac{\alpha_{s} C_{F}}{2 \pi}\left[\frac{1}{\epsilon_{\mathrm{UV}}}+\ln \frac{\mu^{2}}{m^{2}}+\frac{1}{2 \epsilon_{\mathrm{IR}}}\left(\frac{1}{\epsilon_{\mathrm{IR}}}+\ln \frac{\mu^{2}}{m^{2}}\right)+\frac{1}{4} \ln ^{2} \frac{\mu^{2}}{m^{2}}+2+\frac{\pi^{2}}{24}\right] .
$$

It is straightforward to compute the term in the second line of eq. (B.1), and is given as

$$
M_{n}^{m, B}=\frac{\alpha_{s} C_{F}}{2 \pi}\left(\frac{1}{\eta_{+}}+\ln \frac{\nu_{+}}{p_{+}}\right)\left(\frac{1}{\epsilon_{\mathrm{UV}}}-\frac{1}{\epsilon_{\mathrm{IR}}}\right) .
$$

Eq. (6.2) is obtained by combining eqs. (B.3) and (B.4).

\section{B.2 Csoft one-loop calculation with the usoft zero-bin mode $p_{u s}^{\mu} \sim \zeta(m, m, m)$}

In this subsection, we compute the irreducible one-loop contribution to the csoft function $B_{n}$. The naive one-loop contribution $\left(\tilde{M}_{c s}^{m}\right)$ and the zero-bin contribution $\left(M_{c s}^{\varnothing}\right)$ are listed in eqs. (6.16) and (6.17). And the regular contribution with the zero-bin subtraction, $M_{c s}^{m}=\tilde{M}_{c s}^{m}-M_{c s}^{\varnothing}$, can be written as

$$
\begin{aligned}
M_{c s}^{m}= & -\frac{\alpha_{s} C_{F}}{2 \pi} \frac{\left(\mu^{2} e^{\gamma_{\mathrm{E}}}\right)^{\epsilon}}{\Gamma(1-\epsilon)}\left\{\int_{0}^{1} \frac{d x}{x}\left[\int_{0}^{\infty} \frac{d \mathbf{k}_{\perp}^{2}\left(\mathbf{k}_{\perp}^{2}\right)^{-\epsilon}}{\mathbf{k}_{\perp}^{2}+x^{2} m^{2}}-\int_{0}^{\infty} d \mathbf{k}_{\perp}^{2}\left(\mathbf{k}_{\perp}^{2}\right)^{-1-\epsilon}\right]\right. \\
& \left.+\int_{1}^{\infty} \frac{d x}{x} \int_{0}^{\infty} \frac{d \mathbf{k}_{\perp}^{2}\left(\mathbf{k}_{\perp}^{2}\right)^{-\epsilon}}{\mathbf{k}_{\perp}^{2}+x^{2} m^{2}}-\left(\frac{\nu_{+}}{p_{+}}\right)^{\eta_{+}} \int_{1}^{\infty} d x x^{-1-\eta_{+}} \int_{0}^{\infty} d \mathbf{k}_{\perp}^{2}\left(\mathbf{k}_{\perp}^{2}\right)^{-1-\epsilon}\right\} \\
\equiv & M_{c s}^{m, A}+M_{c s}^{m, B}+M_{c s}^{m, C} .
\end{aligned}
$$

Here we divide the integration region of $x$ into $x \in[0,1]$ and $x \in[1, \infty]$. And we denote $M_{c s}^{m, A}, M_{c s}^{m, B}$ and $M_{c s}^{m, C}$ as the contributions from the first, the second and the third terms in the curly brackets respectively.

The contribution $M_{c s}^{m, A}$ for $x \in[0,1]$ involves only the IR divergence since the UV divergence as $\mathbf{k}_{\perp}^{2} \rightarrow \infty$ is cancelled. It is given by

$$
M_{c s}^{m, A}=\frac{\alpha_{s} C_{F}}{4 \pi} \frac{1}{\epsilon_{\mathrm{IR}}}\left(\frac{1}{\epsilon_{\mathrm{IR}}}+\ln \frac{\mu^{2}}{m^{2}}\right) .
$$


The contribution $M_{c s}^{m, B}$ is UV-divergent, and it is given by

$$
M_{c s}^{m, B}=-\frac{\alpha_{s} C_{F}}{4 \pi} \frac{1}{\epsilon_{\mathrm{UV}}}\left(\frac{1}{\epsilon_{\mathrm{UV}}}+\ln \frac{\mu^{2}}{m^{2}}\right) .
$$

The contribution $M_{c s}^{m, C}$ comes from the zero-bin subtraction and includes the rapidity divergence. Since we compute it with on-shell regularization and the massless gluon, the result is the same as eq. (B.4). Finally, combining eqs. (B.6), (B.7) and (B.4), we obtain eq. (6.18).

\section{Acknowledgments}

J. Chay is supported by Basic Science Research Program through the National Research Foundation of Korea (NRF) funded by the Ministry of Education(Grant No. NRF-2019R1 F1A1060396). C. Kim was supported by Basic Science Research Program through the National Research Foundation of Korea (NRF) funded by the Ministry of Science and ICT (Grant No. NRF-2017R1A2B4010511).

Open Access. This article is distributed under the terms of the Creative Commons Attribution License (CC-BY 4.0), which permits any use, distribution and reproduction in any medium, provided the original author(s) and source are credited.

\section{References}

[1] C.W. Bauer, S. Fleming and M.E. Luke, Summing Sudakov logarithms in $B \rightarrow X_{s} \gamma$ in effective field theory, Phys. Rev. D 63 (2000) 014006 [hep-ph/0005275] [INSPIRE].

[2] C.W. Bauer, S. Fleming, D. Pirjol and I.W. Stewart, An Effective field theory for collinear and soft gluons: Heavy to light decays, Phys. Rev. D 63 (2001) 114020 [hep-ph/0011336] [INSPIRE].

[3] C.W. Bauer, D. Pirjol and I.W. Stewart, Soft collinear factorization in effective field theory, Phys. Rev. D 65 (2002) 054022 [hep-ph/0109045] [INSPIRE].

[4] C.W. Bauer, S. Fleming, D. Pirjol, I.Z. Rothstein and I.W. Stewart, Hard scattering factorization from effective field theory, Phys. Rev. D 66 (2002) 014017 [hep-ph/0202088] [INSPIRE].

[5] J.-y. Chiu, A. Jain, D. Neill and I.Z. Rothstein, The Rapidity Renormalization Group, Phys. Rev. Lett. 108 (2012) 151601 [arXiv:1104.0881] [INSPIRE].

[6] J.-Y. Chiu, A. Jain, D. Neill and I.Z. Rothstein, A Formalism for the Systematic Treatment of Rapidity Logarithms in Quantum Field Theory, JHEP 05 (2012) 084 [arXiv: 1202.0814] [INSPIRE].

[7] A.V. Manohar and I.W. Stewart, The Zero-Bin and Mode Factorization in Quantum Field Theory, Phys. Rev. D 76 (2007) 074002 [hep-ph/0605001] [INSPIRE].

[8] A.V. Manohar and I.W. Stewart, Running of the heavy quark production current and $1 / v$ potential in QCD, Phys. Rev. D 63 (2001) 054004 [hep-ph/0003107] [INSPIRE].

[9] A.H. Hoang, A.V. Manohar and I.W. Stewart, The Running Coulomb potential and Lamb shift in QCD, Phys. Rev. D 64 (2001) 014033 [hep-ph/0102257] [INSPIRE]. 
[10] J. Collins, Foundations of perturbative QCD, Camb. Monogr. Part. Phys. Nucl. Phys. Cosmol. 32 (2011) 1, Cambridge University Press (2013) [InSPIRE].

[11] A. Idilbi and T. Mehen, On the equivalence of soft and zero-bin subtractions, Phys. Rev. D 75 (2007) 114017 [hep-ph/0702022] [INSPIRE].

[12] A. Idilbi and T. Mehen, Demonstration of the equivalence of soft and zero-bin subtractions, Phys. Rev. D 76 (2007) 094015 [arXiv: 0707.1101] [InSPIRE]

[13] T. Becher and G. Bell, Analytic Regularization in Soft-Collinear Effective Theory, Phys. Lett. B 713 (2012) 41 [arXiv:1112.3907] [InSPIRE].

[14] Y. Li, D. Neill and H.X. Zhu, An exponential regulator for rapidity divergences, Nucl. Phys. B 960 (2020) 115193 [arXiv: 1604.00392] [INSPIRE].

[15] M.A. Ebert, I. Moult, I.W. Stewart, F.J. Tackmann, G. Vita and H.X. Zhu, Subleading power rapidity divergences and power corrections for $q_{T}$, JHEP 04 (2019) 123 [arXiv: 1812.08189] [INSPIRE].

[16] J.-y. Chiu, A. Fuhrer, A.H. Hoang, R. Kelley and A.V. Manohar, Soft-Collinear Factorization and Zero-Bin Subtractions, Phys. Rev. D 79 (2009) 053007 [arXiv:0901.1332] [INSPIRE].

[17] J. Chay, C. Kim, Y.G. Kim and J.-P. Lee, Soft Wilson lines in soft-collinear effective theory, Phys. Rev. D 71 (2005) 056001 [hep-ph/0412110] [INSPIRE].

[18] P. Jaiswal and T. Okui, Reemergence of rapidity-scale uncertainty in soft-collinear effective theory, Phys. Rev. D 92 (2015) 074035 [arXiv: 1506.07529] [INSPIRE].

[19] S.D. Ellis, C.K. Vermilion, J.R. Walsh, A. Hornig and C. Lee, Jet Shapes and Jet Algorithms in SCET, JHEP 11 (2010) 101 [arXiv: 1001.0014] [InSPIRE].

[20] S. Catani and M.H. Seymour, The Dipole formalism for the calculation of QCD jet cross-sections at next-to-leading order, Phys. Lett. B 378 (1996) 287 [hep-ph/9602277] [INSPIRE].

[21] S. Catani and M.H. Seymour, A General algorithm for calculating jet cross-sections in NLO QCD, Nucl. Phys. B 485 (1997) 291 [Erratum ibid. 510 (1998) 503] [hep-ph/9605323] [INSPIRE].

[22] T. Becher, A. Broggio and A. Ferroglia, Introduction to Soft-Collinear Effective Theory, Lect. Notes Phys. 896 (2015) 1, Springer (2015) [DOI] [arXiv:1410.1892] [INSPIRE].

[23] T. Kasemets, W.J. Waalewijn and L. Zeune, Calculating Soft Radiation at One Loop, JHEP 03 (2016) 153 [arXiv: 1512.00857] [INSPIRE].

[24] T. Becher and M. Neubert, Infrared singularities of scattering amplitudes in perturbative QCD, Phys. Rev. Lett. 102 (2009) 162001 [Erratum ibid. 111 (2013) 199905] [arXiv:0901.0722] [INSPIRE].

[25] T. Becher and M. Neubert, On the Structure of Infrared Singularities of Gauge-Theory Amplitudes, JHEP 06 (2009) 081 [Erratum ibid. 11 (2013) 024] [arXiv:0903.1126] [INSPIRE].

[26] J. Chay and C. Kim, Gauge invariant Lagrangian of the soft collinear effective theory and its application to soft-collinear currents, hep-ph/0401089 [INSPIRE].

[27] A. Mitov and S. Moch, The Singular behavior of massive QCD amplitudes, JHEP 05 (2007) 001 [hep-ph/0612149] [INSPIRE]. 
[28] T. Ahmed, J.M. Henn and M. Steinhauser, High energy behaviour of form factors, JHEP 06 (2017) 125 [arXiv: 1704.07846] [INSPIRE].

[29] J. Blümlein, P. Marquard and N. Rana, Asymptotic behavior of the heavy quark form factors at higher order, Phys. Rev. D 99 (2019) 016013 [arXiv:1810.08943] [INSPIRE].

[30] J.C. Collins, Algorithm to Compute Corrections to the Sudakov Form-factor, Phys. Rev. D 22 (1980) 1478 [INSPIRE].

[31] J.C. Collins, Sudakov form-factors, Adv. Ser. Direct. High Energy Phys. 5 (1989) 573 [hep-ph/0312336] [INSPIRE].

[32] A.K. Leibovich, Z. Ligeti and M.B. Wise, Comment on quark masses in SCET, Phys. Lett. B 564 (2003) 231 [hep-ph/0303099] [INSPIRE].

[33] I.Z. Rothstein, Factorization, power corrections, and the pion form-factor, Phys. Rev. D 70 (2004) 054024 [hep-ph/0301240] [INSPIRE].

[34] J. Chay, C. Kim and A.K. Leibovich, Quark mass effects in the soft-collinear effective theory and $\bar{B} \rightarrow X_{s} \gamma$ in the endpoint region, Phys. Rev. D 72 (2005) 014010 [hep-ph/0505030] [INSPIRE].

[35] C.W. Bauer, F.J. Tackmann, J.R. Walsh and S. Zuberi, Factorization and Resummation for Dijet Invariant Mass Spectra, Phys. Rev. D 85 (2012) 074006 [arXiv:1106.6047] [InSPIRE]

[36] S. Fleming, A.H. Hoang, S. Mantry and I.W. Stewart, Jets from massive unstable particles: Top-mass determination, Phys. Rev. D 77 (2008) 074010 [hep-ph/0703207] [INSPIRE].

[37] S. Fleming, A.H. Hoang, S. Mantry and I.W. Stewart, Top Jets in the Peak Region: Factorization Analysis with NLL Resummation, Phys. Rev. D 77 (2008) 114003 [arXiv:0711.2079] [INSPIRE].

[38] C. Kim, Exclusive heavy quark dijet cross section, J. Korean Phys. Soc. 77 (2020) 469 [arXiv: 2008.02942] [INSPIRE].

[39] L. Dai, C. Kim and A.K. Leibovich, Heavy quark jet production near threshold, in preparation.

[40] M. Neubert, Factorization analysis for the fragmentation functions of hadrons containing a heavy quark, arXiv:0706.2136 [INSPIRE].

[41] M. Fickinger, S. Fleming, C. Kim and E. Mereghetti, Effective field theory approach to heavy quark fragmentation, JHEP 11 (2016) 095 [arXiv: 1606.07737] [INSPIRE].

[42] J. Chay and C. Kim, Collinear effective theory at subleading order and its application to heavy-light currents, Phys. Rev. D 65 (2002) 114016 [hep-ph/0201197] [INSPIRE].

[43] D. Bertolini et al., Soft Functions for Generic Jet Algorithms and Observables at Hadron Colliders, JHEP 07 (2017) 099 [arXiv: 1704.08262] [INSPIRE].

[44] I. Scimemi and A. Vladimirov, Systematic analysis of double-scale evolution, JHEP 08 (2018) 003 [arXiv: 1803.11089] [INSPIRE].

[45] G.P. Korchemsky and A.V. Radyushkin, Renormalization of the Wilson Loops Beyond the Leading Order, Nucl. Phys. B 283 (1987) 342 [InSPIRE].

[46] I.A. Korchemskaya and G.P. Korchemsky, On lightlike Wilson loops, Phys. Lett. B 287 (1992) 169 [INSPIRE]. 